\title{
An Absolute Measurement of Resistance by the Wenner Method
}

\author{
By James L. Thomas, Chester Peterson, Irvin L. Cooter, and F. Ralph Kotter
}

\begin{abstract}
This paper reports the results of a project started in 1929 for the measurement of resistance in terms of length, time, and the permeability of free space. For this method a mutual inductor was constructed whose value was determined within a few parts in a million in terms of its measured dimensions. Resistance was then measured in terms of this inductance and frequency, using Wenner's commutated direct-current method. The results of the measurements give a value of 0.999994 absolute ohm for the unit now maintained at this Bureau.

The dimensions of the mutual inductor were determined in 1938 and again in 1948 . A comparison of the change in inductance, because of drifts in dimensions, with the electrical determinations made on the two dates shows that there has been no appreciable change during the interval in the value of the unit of resistance as maintained at this Bureau. This is the first method that has been devised to test the stability of the unit to a few parts in a million.
\end{abstract}

\section{Introduction}

The unit of electrical resistance now in general use was selected in 1861 by a committee appointed by the British Association for the Advancement of Science. This committee selected as the unit $10^{9}$ cgs electromagnetic units.

As electrical resistance could be measured in terms of standards of length, time, and the permeability of free space, which is assumed to have a value of unity in the cgs electromagnetic system, a standard of resistance was theoretically not necessary. However, accurate resistance measurements in terms of length and time were extremely difficult, and the committee decided to construct standards of resistance that were to be evaluated in terms of the mechanical units.

A few years later the committee decided, in effect, to change the definition of the unit and define it in terms of the standards that had been evaluated in terms of the cgs units. This procedure was analagous to what had been done in the case of the meter. The meter was originally defined as a ten-millionth of the length of the earth's quadrant, and then a standard was con- structed to have this length. Later, the length of the standard was taken as defining the unit. Unfortunately the standards that were selected as defining the unit of resistance were not very stable with time, and therefore such a unit was rather unsatisfactory. In 1878 Rowland [1] ${ }^{1}$ showed that the British Association unit was in error by more than 1 percent, although it was not known how much of this error was a result of a drift in the unit as maintained by standards and how much arose from errors in the original determination of the values of the standards in terms of length, time, and permeability.

To obtain a standard that would not drift with time, the International Congress that met in Chicago in 1893 adopted as the "international ohm" the resistance of a mercury column of specified dimensions. The dimensions were selected to give a resistance as near $10^{9} \mathrm{cgs}$ electromagnetic units as possible at that time. Subsequent measurements showed the international ohm to be high by about 1 part in 2,000 in terms of its intended value in absolute units.

\footnotetext{
Figures in brackets indicate the literature references at the end of this
} paper. 
The definition of the international ohm in terms of a mercury column apparently solved the problem of the stability of standards, as the unit could be realized by means of mercury tubes to a few parts in $10^{5}$. However by the 1920's, absolute measurements of resistance could be made to an equal accuracy, and the mercury ohm became an unnecessary step in the calibration of wire-wound standards that were universally used to maintain the unit. Moreover, it seemed desirable to eliminate the error of 1 part in 2,000 that had been introduced in the unit at the time the dimensions of the mercury ohm were specified. Therefore in 1928 this Bureau, at the suggestion of the American Institute of Electrical Engineers, and with the unanimous approval of a committee representing the large national electrical organizations of this country, recommended the abandonment of the mercury ohm and the return to the absolute ohm as the basic unit. This recommendation was made through its Advisory Committee on Electricity to the International Committee on Weights and Measures, and with its approval steps were taken looking to the change in units as of January 1, 1940. Because of the war the change was not made until January 1, 1948.

In preparation for this change in units national laboratories were asked to carry out "absoluteohm determinations," and several such determinations were made. Two separate groups, using entirely different methods, worked for several years in this Bureau on the problem of measuring standard resistors in terms of the absolute unit of resistance. The results obtained by one group have already been published, and their results, as well as those obtained by other investigators, are summarized in a paper published in 1944 in this journal [2].

The method chosen for the determination herein reported was suggested in 1908 by Frank Wenner [3], but no measurements were made at that time. His method utilizes direct current flowing through a resistor and the primary of a mutual inductor. The current through the primary of the inductor is reversed periodically, the secondary emf being rectified and balanced against the constant potential difference across the resistor. During the decade following World War I, Wenner, with various assistants, devoted some time to the development of certain aspects of the problem, particularly that of obtaining a constant-speed drive.
Work on the modern development of the method was started in 1929 by Wenner and Peterson, and during the period 1929-33 a fairly satisfactory solution of the constant-speed problem was worked out utilizing a direct-current motor drive with a small 1,000-c/s generator on its shaft. The system was synchronized against a $1,000-\mathrm{c} / \mathrm{s}$ signal obtained from crystal oscillators. During this period many of the measuring circuit components were constructed, the porcelain form for the inductor was procured, and the method as a whole tested, using for the mutual inductor a preliminary model, of the Campbell type, wound on wooden forms. The electrical balances obtained during these trials were quite unsteady, mainly because of erratic brush operation and inability to hold the current through the test resistor constant during primary commutation. Work on the project was allowed to lapse in 1933, but was recommenced at an accelerated pace in 1935 by a group consisting of Frank Wenner, James L. Thomas, Irvin L. Cooter, and F. Ralph Kotter. They improved the speed control [4] largely through a substitution of electronic for mechanical relays. They constructed a mutual inductor of a modified Campbell type and obtained a highly satisfactory performance of the measuring circuit by including a special "reversing generator" in series with the primary of the inductor. A progress report was prepared in September 1938, and a preliminary report of numerical results was issued in December 1938. These reports were issued in mimeograph form, and copies were sent to the Advisory Committee on Electricity, which had been instructed by the International Committee on Weights and Measures to recommend the best value for the ratio of the international and absolute ohms. The necessity of submitting results to the Advisory Committee by the end of 1938 curtailed some phases of the investigation, and a critical study of the results could not be made before issuance of the reports. It was therefore decided to repeat all measurements, after making improvements that the first determinations had shown to be desirable, and then to publish in detail the methods and results of the second investigation. This project was in abeyance during World War II, but was resumed in 1946 by the authors of this paper, and the results are reported herein.

Throughout this paper the abbreviation cgsm will be used to denote that the unit is expressed in 
the cgs electromagnetic system of units. When the magnitudes of small corrections are expressed in proportional parts, the abbreviation ppm, meaning parts per million, will frequently be employed.

\section{Theory of the Method}

The method consists in measuring a resistance in terms of a mutual inductance and a speed of rotation. The mutual inductance is calculated from the dimensions of a mutual inductor, and the speed is held constant by an electronic speed control. The fundamentals of the procedure may be understood from figure 1. By means of the storage battery, $B$, a direct current, $I$, is passed through the resistor, $R$, and the primary of the mutual inductor, $M$. A rotary switch, $C$, reverses the connections to the primary but does not reverse the current through the resistor. This switch is driven at constant speed, the current through the primary of the mutual inductor being reversed about 45 times per second. A commutator, $D$, on the same shaft rectifies the alternating emf induced in the secondary. This rectified emf is connected through a direct-current galvanometer, $G$, to oppose the potential difference across resistor $R$. Assuming that the

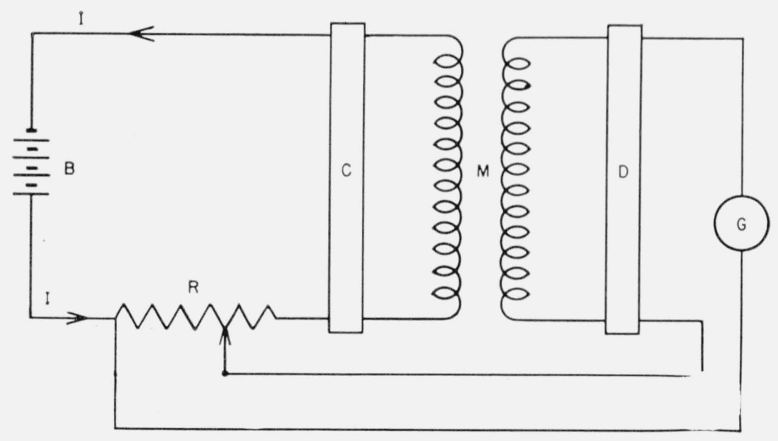

Figure 1. Elementary circuit diogram.

galvanometer can integrate properly, the average induced emf from the mutual inductor and the average potential difference across the resistor will be equal when the galvanometer shows no deflection. Under these conditions

$$
I_{a} R=4 n i_{x} M,
$$

where $I_{a}$ is the average current in the resistor whose resistance, $R$, is to be measured, $2 n$ is the number of reversals per second, $i_{x}$ is the current in the primary of the mutual inductor at the instant of reversal of the secondary connections, and $M$ is the mutual inductance of the inductor. Equation 1 may be expressed in the form

$$
R=4 n M \frac{i_{x}}{I_{a}}
$$

The current ratio $i_{x} / I_{a}$ in this equation must be constant and known with the same accuracy as that desired for $R$. It is extremely difficult to measure a ratio of this kind with an accuracy of $1 \mathrm{ppm}$ or better. The necessity for measuring this current ratio is avoided by so arranging the circuit that the instantaneous value of current in the resistor $R$ never departs from its value averaged over several cycles of operation by as much as $1 \mathrm{ppm}$. This means that during that portion of the cycle when the primary of the mutual inductor is in series with $R$, the current in the inductor is made to assume a value equal to that in the resistor, and since the secondary connections are reversed during this portion of the cycle, $i_{x}$ will consequently be within 1 ppm of $I_{a}$ and the ratio $i_{x} / I_{a}$ will differ from unity by a negligible amount. Inasmuch as the current in the primary of the inductor is very constant when the secondary connections are reversed, the secondary voltage due to induction from the primary will then be negligible and no such emf will be lost during the brief periods when the secondary is shortcircuited. Under these conditions eq 2 becomes

$$
R=4 n M \text {. }
$$

As $M$ is calculated from dimensions measured with reference to length standards, and as $n$ is measured indirectly in terms of the mean solar second, this method permits $R$ to be measured in "absolute" units, that is, in terms of length, time, and permeability. Although permeability does not appear explicitly in eq 3 , it is involved implicitly in $M$, and measurements must be made to ascertain that no material used in the inductor or its immediate field has a permeability that differs from the standard (air) by more than a tolerable amount.

In order that the conditions stipulated in the preceding paragraph may be met, several devices must be added to the simple circuit indicated in figure 1. Iron-cored choke coils placed in the primary circuit tend to maintain the current constant but, since the inductance of an iron-cored 
choke coil is reduced by direct current in the coil, it is difficult to obtain the necessary "smoothing" in this way. In order to overcome this difficulty, what might be called "electronic chokes" are employed, the details of which have been described in another paper [5].

In addition to the iron-cored and electronic chokes, a special device which, for want of a better name, may be called a "reversing generator", is used in the primary circuit. The function of this reversing generator is twofold. In the first place, while the primary circuit is shorted at the reversing commutator, it reverses the current through $M$ in a predetermined manner in order to facilitate the maintenance of small instantaneous emf's in the secondary circuit. The second and more important function of the reversing generator is to supply an emf in the primary circuit at all times equal and opposite to the emf across the primary of the mutual inductor. To the accuracy with which this equality is maintained, the potential difference across the circuit at the reversing switch, $C$, is always zero. Hence the connection or removal of a short-circuit across this part of the circuit does not affect the primary current $I$, as the short-circuit is between points at equal potential.

In order that the galvanometer shall properly integrate the current in the secondary circuit, it is necessary to prevent the passage of large currents through it. This is done by inserting very large choke coils and an inductor generator in series in the secondary circuit. The choke coils tend to reduce the alternating component of current through the galvanometer without greatly reducing the unidirectional current resulting from a lack of balance. An inductor generator has an emf that always averages zero, and hence it has no effect on the balance of the circuit. If it is so designed that its emf is at every instant equal and opposite to the sum of the $I R$ drop and the rectified secondary induced emf at balance, the instantaneous values of the net emf in the secondary circuit can be kept very small.

It was not feasible to design the inductorgenerator in the secondary circuit to give a comparatively complicated wave form to match that produced in the circuit by the sum of the $I R$ drop and the secondary-induced emf. The order of the design was therefore reversed, the inductorgenerator being built to give an approximately rectangular-shaped emf wave. Then the primary reversing-generator was designed to reverse the current through the primary of the mutual inductor in such a way that the secondary emf so induced, when added to the $I R$ drop through the primary resistor, would give a voltage equal and opposite at every instant to that of the inductor generator. The accuracy of matching was not carried to an extreme, it being intended that the inductor-generator should reduce the voltage amplitude by a factor of about 10 .

Besides the design and construction of the apparatus just mentioned for the measurement circuit it was necessary to design, construct, measure, and calculate the inductance of a mutual inductor, and it was also necessary to develop a system of speed control such that the reversing commutators could be driven at a constant known rate. The work on the mutual inductor, speed control, and on the measuring circuit will be described under separate headings.

\section{Construction of the Mutual Inductor}

\section{General Considerations}

The value of mutual inductance required for this circuit was fixed by the values selected for $R$ and $n$. For $R$ a double-walled 1-ohm resistor was used, as this type was the most stable in resistance of any available. A value was selected for $n$ that would not be an integral multiple or submultiple of the frequency of any probable extraneous emf. The value of about $23 \mathrm{rps}$, actually 1000/44, was selected on the basis of the frequencies of power lines and a-c generators known to be in operation in the vicinity of the equipment. These values selected for $R$ and $n$ required the use of 11 millihenries of mutual inductance. However, an inductance of slightly less than 11 millihenries was used in order that the circuit could be balanced by means of a shunt of moderate size across the 1-ohm standard resistor.

An excellent design for an inductor of approximately this value was available as the result of work by Campbell [6] at the National Physical Laboratory. This inductor consists of a singlelayer helix on an accurately constructed cylinder, and a multilayer secondary in such a position that its dimensions need not be accurately known. In order to fulfill this condition, Campbell divided his primary winding into two coaxial single-layer coils 
with a considerable distance between them. A current flowing through the two coils connected in series aiding gave a circle of zero magnetic field outside the primary cylinder, and the secondary coil was located about this circle. Near the circle of zero field the space rate of change of field strength was small, and errors in measurements of the location of the secondary turns gave negligible errors in the calculated flux linked with the secondary turns and hence in the calculated mutual inductance.

For the present investigation it seemed desirable to have the resistance and inductance of the secondary small, as it was to be shorted during commutation. It was decided, therefore, to use only about half as many turns on the secondary as on the primary. Also, instead of following Campbell's design exactly, it was decided to try to increase the volume of the region of low field strength in which the secondary was to be located, thus allowing the use of a secondary winding of larger cross section and hence larger wire. It appeared that this increase might be obtained by breaking the primary into three separate sections. With a current through the three sections connected in series aiding, it was expected that two external circles of zero field strength would be obtained. By properly selecting the number of turns and the relative positions of the three sections, it appeared possible to bring the two circles of zero field close together so that between them a region of very small field would result, in which the secondary winding could be located. The calculation of the fields for a considerable number of tentative designs would have been very laborious, so the investigation of the fields was first carried out experimentally.

\section{Experimental Design of Mutual Inductor}

To investigate the fields experimentally for different combinations of turns on the primary windings, a full-scale model was made of the proposed primary. A wooden cylinder $41 \mathrm{~cm}$ in diameter and $90 \mathrm{~cm}$ in length was wound from one end to the other with copper wire, the pitch of $2 \mathrm{~mm}$ being the same as that to be used on the primary of the standard inductor. By soldering two short-circuiting jumpers between turns, the practical equivalent of three separated coaxial coils of the same diameter was obtained. The resulting coil arrangement is shown in section in figure 2. A current flowing through coils $A$ and $B$ in series will give a zero field in the neighborhood

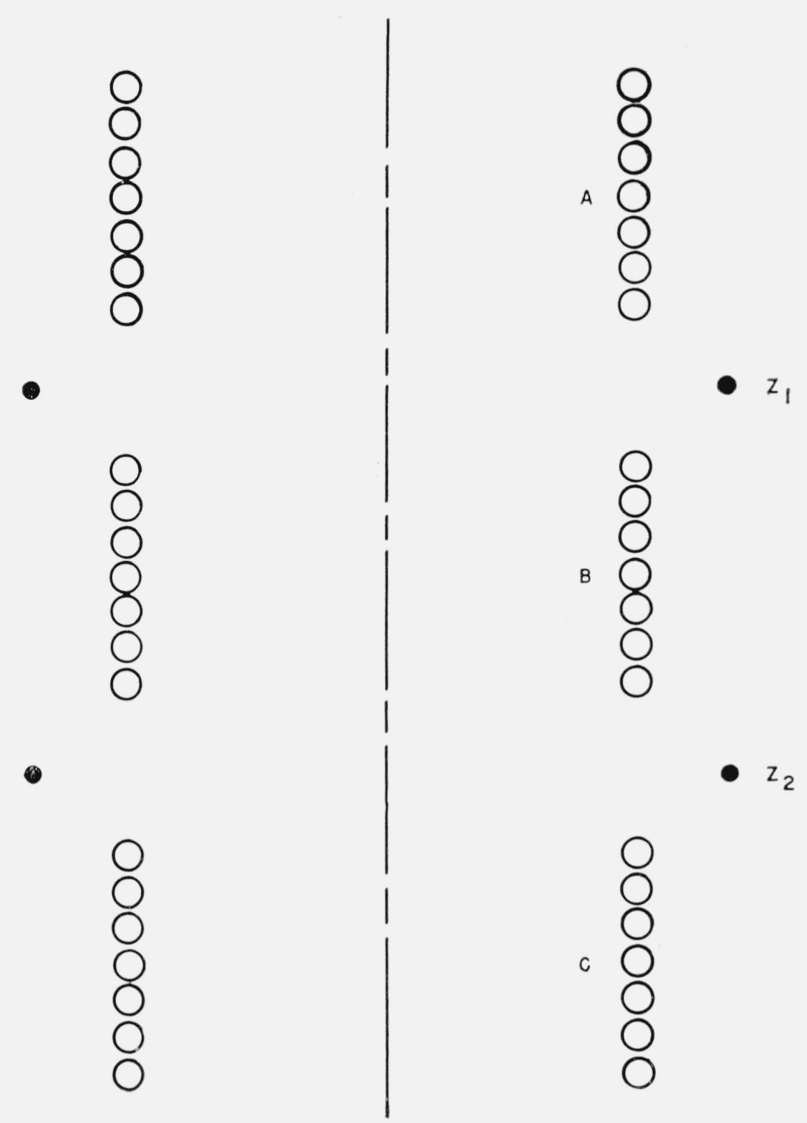

Figure 2. Diagram illustrating the approximate location of two major zero circles surrounding a three-section helix.

of $Z_{1}$. Likewise, a current through $B$ and $C$ in series will give a zero field at $Z_{2}$. With current through all three coils in series, two circles of zero field will be obtained but in somewhat different positions than for the separate combinations of two coils. If now the coil $B$ is shortened, it would be expected that $Z_{1}$ and $Z_{2}$ would approach each other and probably change their radial distance from the axis. The experimental design of the inductor consisted in locating the positions of the zero fields and finding what lengths and relative positions of the coils would bring the two zero circles close together with a fairly weak field surrounding them.

To locate the positions of the zero circles for the different combinations of coils, a small magnet and mirror were attached to a fine phosphorbronze wire. The length and tension in the wire were adjusted until the resonant frequency of the magnet and mirror was $60 \mathrm{c} / \mathrm{s}$. This "magnetom- 
eter" was then used to explore the field of the primary of the inductor while it was carrying an alternating current of a frequency of $60 \mathrm{c} / \mathrm{s}$.

The fields of a number of coil combinations were examined. In each combination the two end coils always had the same number of turns and the two blank spaces were always equal, so that there was symmetry about the center. As had been anticipated, the zero circles approached one another as the center section was shortened. It was found, however, that with the center section very short both zero circles were in a plane normal to the axis and passing through the center of the middle coil, but their radii were no longer equal. As the length of the center section was further decreased, one zero moved away from and the other toward the helix.

With the coil combination that was finally selected, the two zero circles were 4.5 and $6.5 \mathrm{~cm}$, respectively, from the surface of the primary. For the $41-\mathrm{cm}$ form this required 159 turns in each end section and 25 turns in the central section. A blank space corresponding to 28 turns was left at each end of the central section. The pitch was $2 \mathrm{~mm}$. After this design had been determined experimentally, it was checked by calculation before the secondary coil form was constructed. These calculations verified the locations of the zero circles and showed the field to be very weak between them and for a reasonable distance in the axial direction. The secondary winding form was made with a channel rectangular in section, having a width of about $8 \mathrm{~mm}$ in the direction of the axis of the primary and $2.5 \mathrm{~cm}$ in the direction of its radius. The general arrangement and proportion of the final design of the inductor is shown in section in figure 3. Each śmall open circle represents about five active primary turns. The two dots within the secondary winding channel represent the two circles where the field strength of the primary is zero.

The experimental work described in connection with the design of the modified Campbell-type inductor was performed in 1936. Postwar reconsideration of this work has indicated that the space surrounding a multisection helix may contain more than two major circles of zero magnetic field strength and that some of these may have been overlooked in the original investigation. This matter may be reexamined at a later date, but for the present it is sufficient to state that the design adopted as a result of the work described has proved satisfactory.

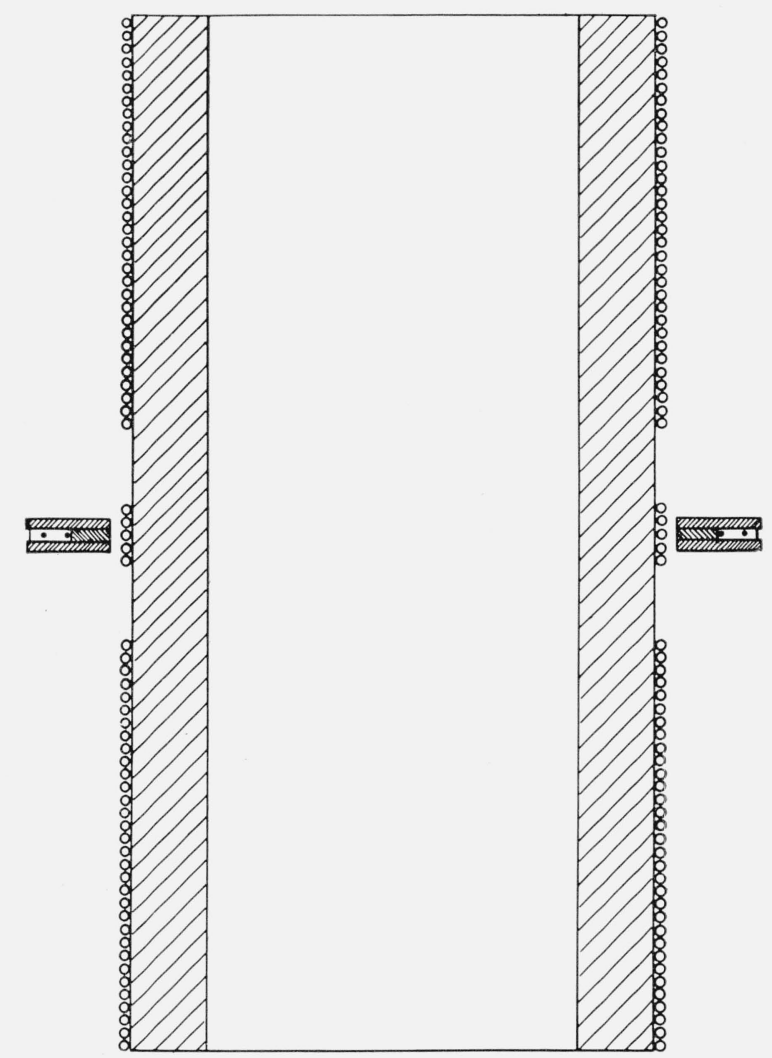

Figure 3. Arrangement and general proportions of the mutual inductor.

\section{Construction of the Primary}

(a) Preparation of the Porcelain Form

The primary of the standard mutual inductor was wound on a porcelain ${ }^{2}$ cylinder $41 \mathrm{~cm}$ in diameter and $83 \mathrm{~cm}$ in length. The wall thickness when completed was about $6 \mathrm{~cm}$. The first step in the construction was the rough grinding of the inside and outside of this cylinder until an approximately uniform wall thickness was obtained. The cylinder was then mounted on a rotating spider and lapped until the outside diameter was uniform to about $0.005 \mathrm{~mm}$. It was then mounted. in a lathe and a shallow 2-mm thread was cut in the surface with a diamond-charged steel wheel. The ends of the cylinder were then cut off square using the same diamond-charged wheel mounted on the cross feed of the lathe.

Lapping blocks were made to match the rough thread that had been cut in the porcelain cylinder

\footnotetext{
${ }^{2}$ This cylinder was made by the Locke Insulator Co. of Baltimore, Md., especially for this job, using the wet process for making insulator porcelain.
} 
by cutting a $V$-shaped thread on the inside of a piece of heavy-walled brass pipe about $41 \mathrm{~cm}$ in diameter. The pitch of this thread was $2 \mathrm{~mm}$, the same as that on the porcelain cylinder. The threaded brass pipe was cut into pieces about $3 \mathrm{~cm}$ square, and these were cemented to steel frames to form laps for finishing the threads on the cylinder. The procedure was practically identical with that described in detail by Curtis, Moon, and Sparks [7].

Three separate lapping frames were used. The first frame was half the length of the porcelain cylinder, and the lapping blocks were attached in four separate groups. One set was attached at each end of the frame, a third about an inch from the center of the frame, and the fourth group was attached at about 2 in. from one of the end groups. During lapping, the lap was frequently turned end-for-end, and it is believed that the nonsymmetrical spacing of the lapping blocks reduced the tendency to cause a low region near the center of the lapped cylinder.

A long lap will make the cylinder straight and the thread remarkably uniform in pitch, but there is a tendency to make the diameter slightly small near each end and at the center. This can be corrected by using a second, very short lap and working locally to reduce the diameter in the regions where the lapping is least. Such a short lap was constructed and used occasionally. To obtain an even more uniform diameter, a third lap was constructed. The frame for this lap reached entirely around the cylinder and carried a number of lapping blocks, in a circle on the inner face, which pressed against the cylinder. This frame was not very rigid, and the circumference could be reduced by means of setscrews, thus pulling the blocks down tight against the cylinder. This "nut" lap was very effective in maintaining a uniform diameter, and it was used alternately with the other two laps. At the completion of the lapping, the diameter was probably uniform to about $0.001 \mathrm{~mm}$, and no variation in pitch could be detected with the apparatus that was used for the preliminary measurements. The uniformity of the diameter did not remain after the wire had been wound onto the cylinder. The tension in the wire caused a considerable compression of the porcelain form, and the compression was not uniform. This nonuniform compression resulted partly from the fact that the porcelain was only partially vitrified and the vitrification was probably not uniform in depth. The interior of the porcelain had been purposely left unvitrified in order to increase the strength of the cylinder.

At the completion of lapping of the groove in the porcelain cylinder, 4-mm holes were drilled through the form at the six positions where it was desired to end the three sections of the winding. These holes were made with drills having tips of tungsten carbide, using an ordinary drill press. Kerosine was continuously supplied along the drill, and the drills needed resharpening after each centimeter or so of penetration.

After the holes had been drilled, the approach sides were cut away so as to avoid sharp bends of the wire near the holes. This was done by manually drawing back and forth a copper wire charged with silicon carbide. Short brass rods were made, which fitted snugly into the drilled holes. One end of each rod was slotted to permit a tapered screw to spread the end of the rod and clamp it securely in the hole through the porcelain. These rods served as anchors for the wire of the primary winding. Holes for the ends of the wire of the primary winding were drilled through the rods near the outer ends, so the wires could be attached by set screws and soft solder.

\section{(b) Winding of the Primary}

After the lapping and the drilling had been completed, the porcelain cylinder was mounted on a wooden mandril for winding. This mandril had an outside diameter about $2 \mathrm{~cm}$ less than that of the inside diameter of the cylinder. The entire surface of the mandril was first covered with a continuous helical winding of thin-walled rubber tubing which lay flat against the mandril when connected to a vacuum line. After the cylinder had been placed on the mandril, air was again admitted into the rubber tubing, which expanded and supported the porcelain cylinder on a soft cushion. The mandril and cylinder were then mounted so that they could be turned at a rate of about $5 \mathrm{rpm}$ by means of an electric motor.

During winding, the wire was pulled through a die directly onto the grooved cylinder. The drawing tension was obtained from the motor that rotated the cylinder. The die was so mounted that it moved along $2 \mathrm{~mm}$ for each turn of the porcelain cylinder and thus remained always alined with the groove on the cylinder. A 
guide consisting of a smooth hole in a maple block lined up the wire on the entrance side of the die. The carriage for the die was at a distance of about $3 \mathrm{~m}$ from the cylinder. In this space the wire was carefully washed after it had been drawn but before it reached the porcelain cylinder.

The wire was of annealed high-purity oxygenfree copper, with an initial diameter of $1.85 \mathrm{~mm}$. Two preliminary drawings were made through dies of 1.77 and $1.72 \mathrm{~mm}$, and the final drawing onto the porcelain cylinder was through a 1.70$\mathrm{mm}$ die. All three of the dies were made of tungsten-carbide. There was no untapered portion to the die, and the wire was continuously reduced in diameter until the minimum was reached. With the speed of drawing that was used, this shape was found to be very effective in maintaining a lubricating film on the wire.

During the drawing, the dies were mounted in a housing that was belted to an electric motor and rotated at a rate of about $1,200 \mathrm{rpm}$. This housing was the same as that which was used in making and finishing the openings in the dies, and the holes were well alined with the axis of rotation of the housing. The rotation of the dies accomplished two purposes. In the first place, it avoided the possibility of the wire's being slightly out-of-round and lying in a definite orientation with reference to the grooves on the porcelain cylinder. In the second place, the rotation was found to assist ir: maintaining lubricant between the die and the wire. In fact, with the linear speed of about 10 $\mathrm{cm} / \mathrm{s}$ for the drawing of the wire, and a rotation of the die of $1,200 \mathrm{rpm}$, the film of lubricant was so well maintained that there was apparently no actual direct contact between the wire and the die. This permitted the drawing of the wire without wear on the die and there was no measurable difference in the diameter of the wire at the starting and at the finishing end of the coil, measurements being made to about $\pm 0.2 \mu$.

In order to draw without contact between the wire and the die, at the speed that was used, it was necessary to make a special lubricant. This lubricant was a soft soap made by boiling down a mixture of linseed oil soap and rosin soap until a thick sticky paste was obtained.

After drawing, the wire was held in place by means of a clamp near the end of the cylinder. The end of the wire was then passed through the hole in the anchor rod and held in place by means of the set screw and solder. The rod was then forced into the hole in the porcelain until the wire was put under a considerable tension, after which the rod was fastened in place by means of the expansion screw. After the ends had been attached, the wire was clamped and then cut at the two places needed to separate the winding into three parts. The three parts were separated by removing three turns at each cut, and the ends were attached to brass anchor rods as described above. When completed, no part of the anchor rods extended through to the inside nor to the outside of the porcelain cylinder, and the avoidance of sharp turns at the ends of the wire left the outside of the inductor smooth.

\section{Construction of the Secondary}

The secondary form was constructed of three annular disks cut from sheets of heavy plate glass. All three disks have the same inner radius of $21.5 \mathrm{~cm}$, whereas two disks have outside radii of $28 \mathrm{~cm}$, and the third disk has an outside radius of only $25 \mathrm{~cm}$. The three disks were cemented together, with the smallest placed between the two large disks to give a winding form of the shape shown in figure 4 . The channel thus formed is about $8 \mathrm{~mm}$ wide and $3 \mathrm{~cm}$ in depth, and the secondary coil was wound in this space.

After the glass disks had been cut roughly to size they were mounted in a lathe and cut accurately with a diamond-charged steel disk.
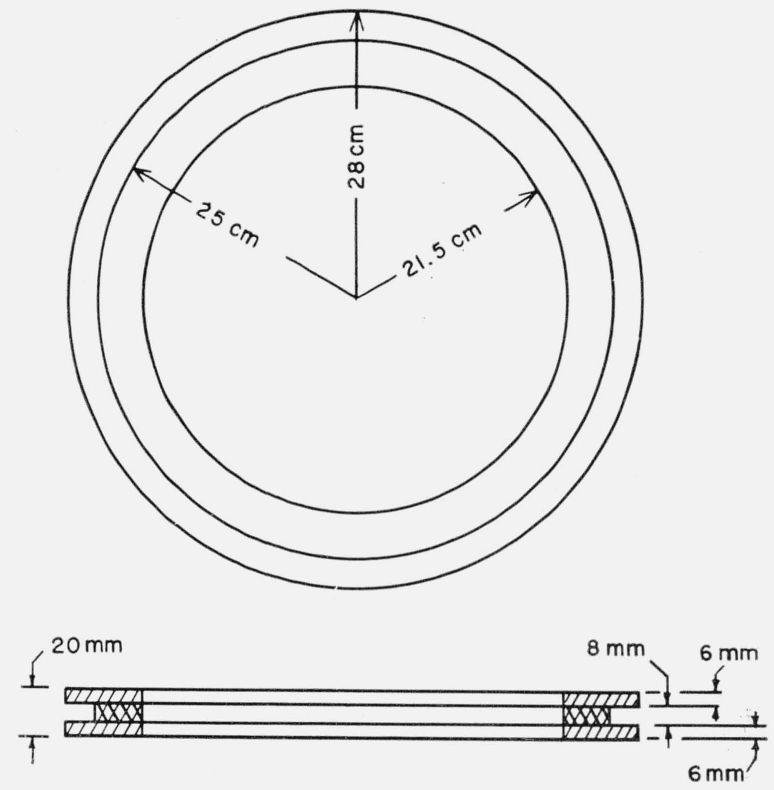

Figure 4. Approximate dimensions of glass form for secondary winding. 
The glass disks were cemented together with a cement that was made by stirring together equal volumes of powdered glass sand and 40 percent solution of sodium silicate. While the cement was allowed to thoroughly harden, which required several days, the channel was kept uniform by means of spacing blocks that were slightly thicker than the inner disk.

The wire used in winding the secondary coil was No. 20 AWG silk-enameled copper. There are 24 layers with $823 / 24$ turns per layer, and three turns in a twenty-fifth layer, giving a total of 218 turns. Between each layer and the next was wound a strip of strong paper, $0.15 \mathrm{~mm}$ in thickness. By dropping back one- twenty-fourth turn instead of winding nine full turns on each layer, it was possible to reduce the distortion caused in passing from one layer to the next.

The design of the mutual inductor is such that there is little difference in the contribution to the total mutual inductance of a turn in any layer and the corresponding turn in the next adjacent layer of the secondary winding. Hence the exact shape and position of the wire as it passes from one layer to the next is of minor importance. No difference exceeding 1 or 2 parts in $10^{7}$ of the mutual inductance would be obtained if these cross-over sections of wire were considered to be entirely in either of the adjacent layers instead of being in intermediate positions, as is the actual case.

\section{Electrical Connections}

When in use, the primary of the mutual inductor is supported from one end, with its axis vertical, and of course this is also its position when its dimensions are measured. The secondary is suspended by means of small brass wires from glass strips laid across the top of the primary. A side view of the finished inductor is shown in figure 5 . The twisted pair of conductors forming the secondary leads may be seen on the right. Connections to the primary are made along the front of the cylinder, which appears at the left of this photograph. In the vicinity of the inductor the primary leads are in the form of a coaxial cable. The outer conductor of this cable makes connection at the bottom of the primary winding. The inner conductor extends vertically to the top of the cylinder where it makes connection. The three sections of the primary are connected in series by means of two "jumpers" of the type shown in figure 6 .

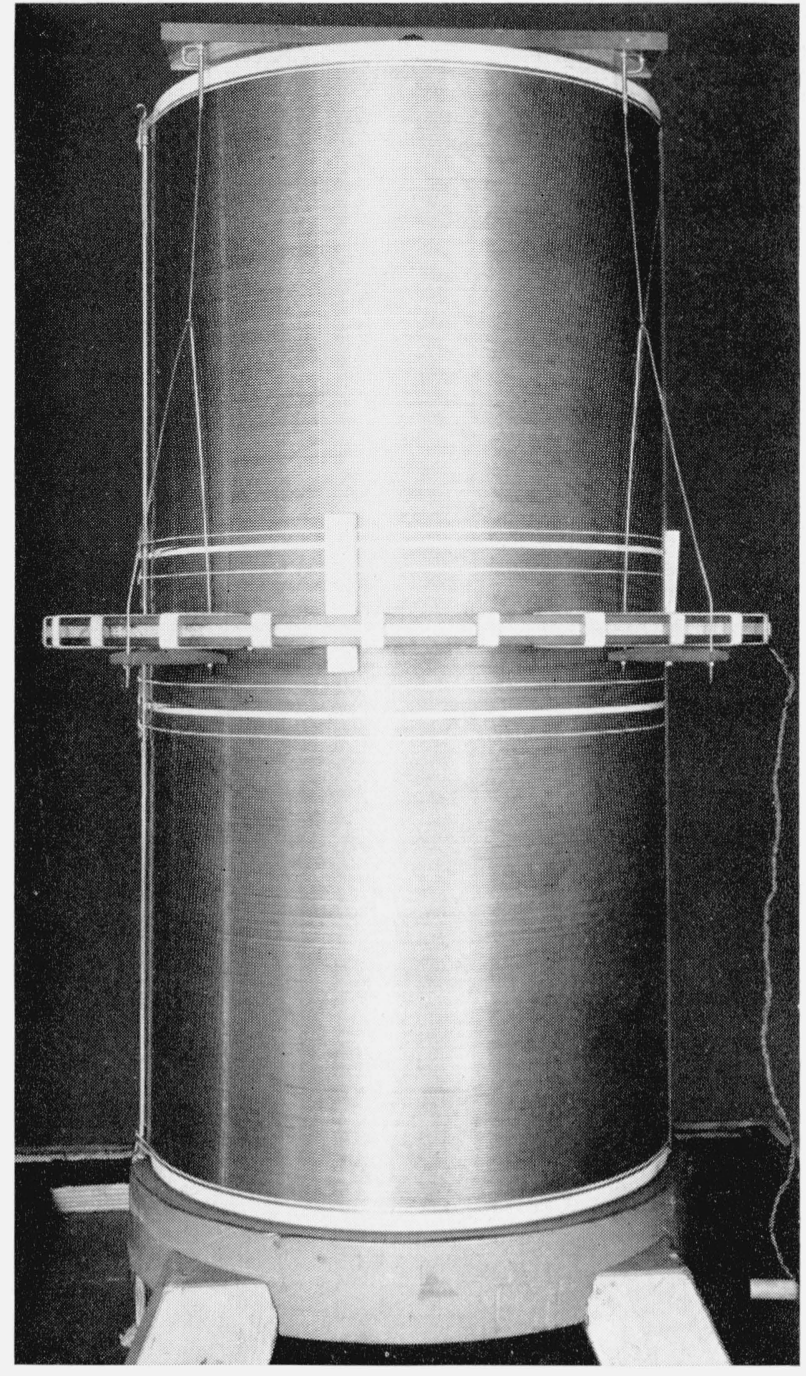

Figure 5. General view of the finished inductor.

Each jumper consists of an 8-mm brass tube 65 $\mathrm{mm}$ long, slit longitudinally to reduce eddy currents. Copper contacts, $K$ and $K^{\prime}$, attached near the ends of the tube, press against the appropriate wires to connect two coils. One contact is grooved to maintain its position vertically, while the other is flat. Amber bushings pressed into each end of the tube are drilled along the axis with $1.7-\mathrm{mm}$ holes, and the vertical conductor passes through these holes. The foot, $F$, extends at right angles to the tube and prevents any appreciable rotation from the proper position for the contacts. The connectors are held tightly in place by means of several turns of linen thread around the primary cylinder.

Similar contact blocks are used at the extreme 


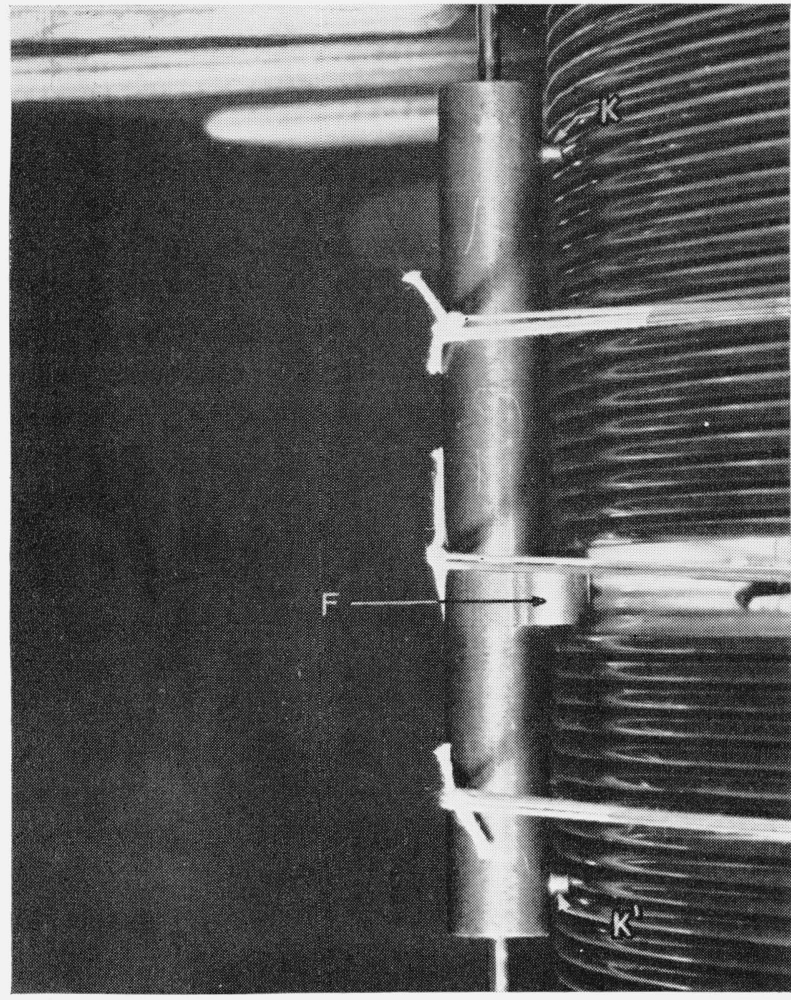

Figure 6. Close-up view of a jumper used to connect adjacent sections of the primary.

ends of the winding for connections to the primary lead wires. These blocks are much shorter, and each has only one contact.

\section{Measurements of the Dimensions of the Inductor}

\section{Measurements of the Primary}

(a) General Considerations

For the measurements of the dimensions of the primary of the mutual inductor a special comparator was built, and assembled in an underground room. There a triangular-shaped concrete pier was available, which was supported by the undisturbed earth under the room, with no direct connection to the floor. This triangular pier is about $1.5 \mathrm{~m}$ on the side, $10 \mathrm{~cm}$ thick, supported at each corner by a concrete footing.

The comparator was designed to be mounted on this pier. At each corner of the pier was placed a vertical hexagonal steel post. These posts are a little over $2 \mathrm{~m}$ in length and $6.5 \mathrm{~cm}$ between opposite flat surfaces. A welded steel framework made of 4 by $6 \mathrm{~cm}$ seamless rectangular steel tubing with $6-\mathrm{mm}$ wall thickness was bolted to

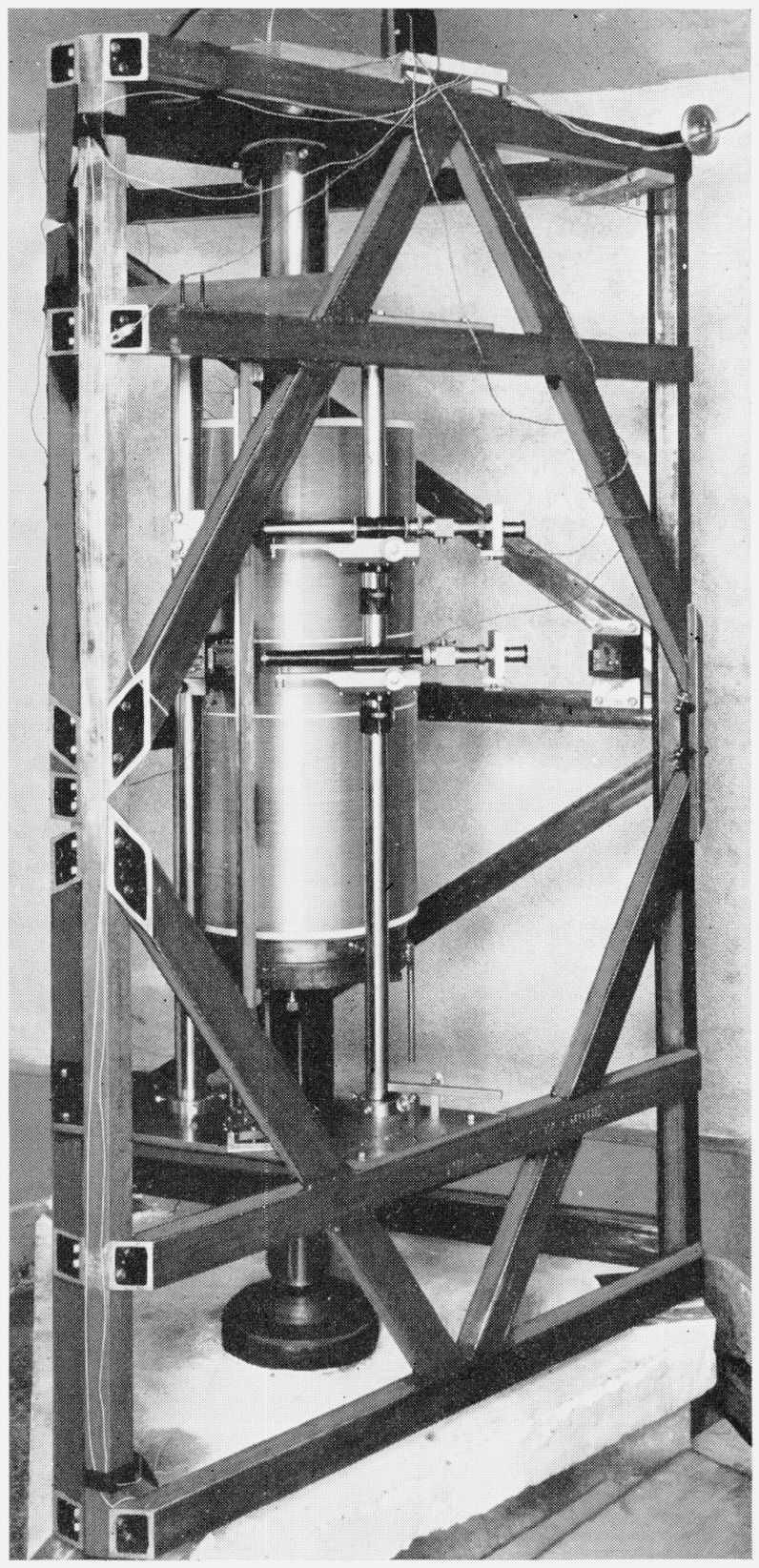

Figure 7. General view of the comparator, arranged for making pitch measurements.

these three corner posts. The details of the construction are shown in figure 7. A triangular piece of sheet steel $1 \mathrm{~cm}$ thick, stiffened by welded steel ribs, was bolted to the top of the framework. At the center of this triangular piece, on the lower side, was welded a short piece of heavy steel tubing that carries four setscrews for adjusting to the vertical a threaded iron tube on which is carried the porcelain cylinder to be measured. This iron 
tube rests on a steel ball, which allows a free adjustment of the position of the top end.

The threaded iron tube was made from a piece of centrifugally cast iron pipe of 12 -cm outside diameter and $2.5-\mathrm{cm}$ wall thickness. This was first mounted in a precision lathe and turned smooth on the outside. A 2-mm thread was then turned in the tubing for considerably over half its length. When mounted in the comparator frame,

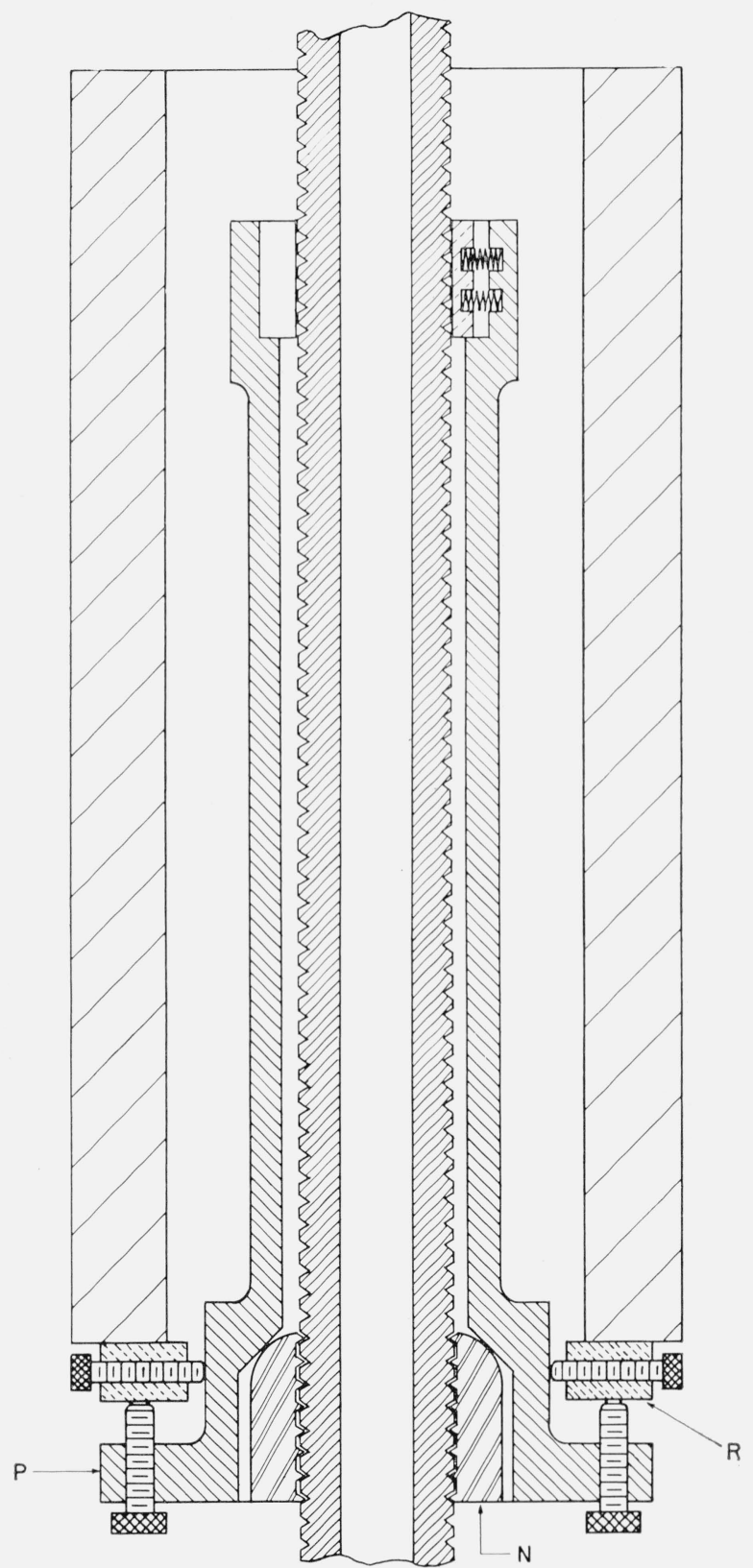

Figure 8. Platform for supporting primary cylinder in comparator. the tube is held in a vertical position with the threaded part at the bottom. A bronze nut on the screw supports a cast iron platform that is used in turn to support the porcelain cylinder. By rotating the porcelain cylinder and platform, friction causing the nut to also turn, the cylinder can be moved up and down in the comparator frame. Since the thread on the screw and that lapped on the cylinder are of the same pitch, the raising and lowering of the cylinder will bring all the turns of the cylinder into similar positions with reference to the measuring parts of the comparator.

A cross section showing the porcelain cylinder supported on the screw is shown in figure 8 . The cylinder rests on the cast iron ring, $R$, which in turn is supported by four large screws so that the level of the cylinder is adjustable. Moreover, the radial position of the porcelain cylinder is adjustable by four screws, which pass through threaded holes in the cast iron ring and press against the wall of the supporting platform $P$. The weight of the platform and its load is carried on the rounded top of the bronze nut, $N$, through the $45^{\circ}$ tapered shoulder, as shown. This circular area of contact centers the bottom end of the platform without binding. At the top of the platform housing is a bearing that consists of three smooth bronze segments that rest against the threads of the screw. The bearing surfaces are spaced $120^{\circ}$ and two are fastened permanently in place. The third segment is movable in a radial direction and is held snugly against the screw by means of springs. This type of construction was used in order to avoid the necessity of making a very long nut or of alining two separate nuts.

After the cylinder is mounted in the comparator, the screw and cylinder are alined as follows: The screw is first set vertical, and this condition is attained when a sensitive spirit level on top of the porcelain cylinder does not change its reading as the cylinder is rotated. By adjusting the cylinder supports until this constant reading is "level", the porcelain cylinder is also set vertical. The radial adjustment of the cylinder is judged by means of a block that slides along a way until it comes in contact with a wire on the cylinder. An index mark on this block can be read through a telescope with a micrometer eyepiece, and adjustments are made until the reading changes by a minimum amount as the cylinder is rotated. 


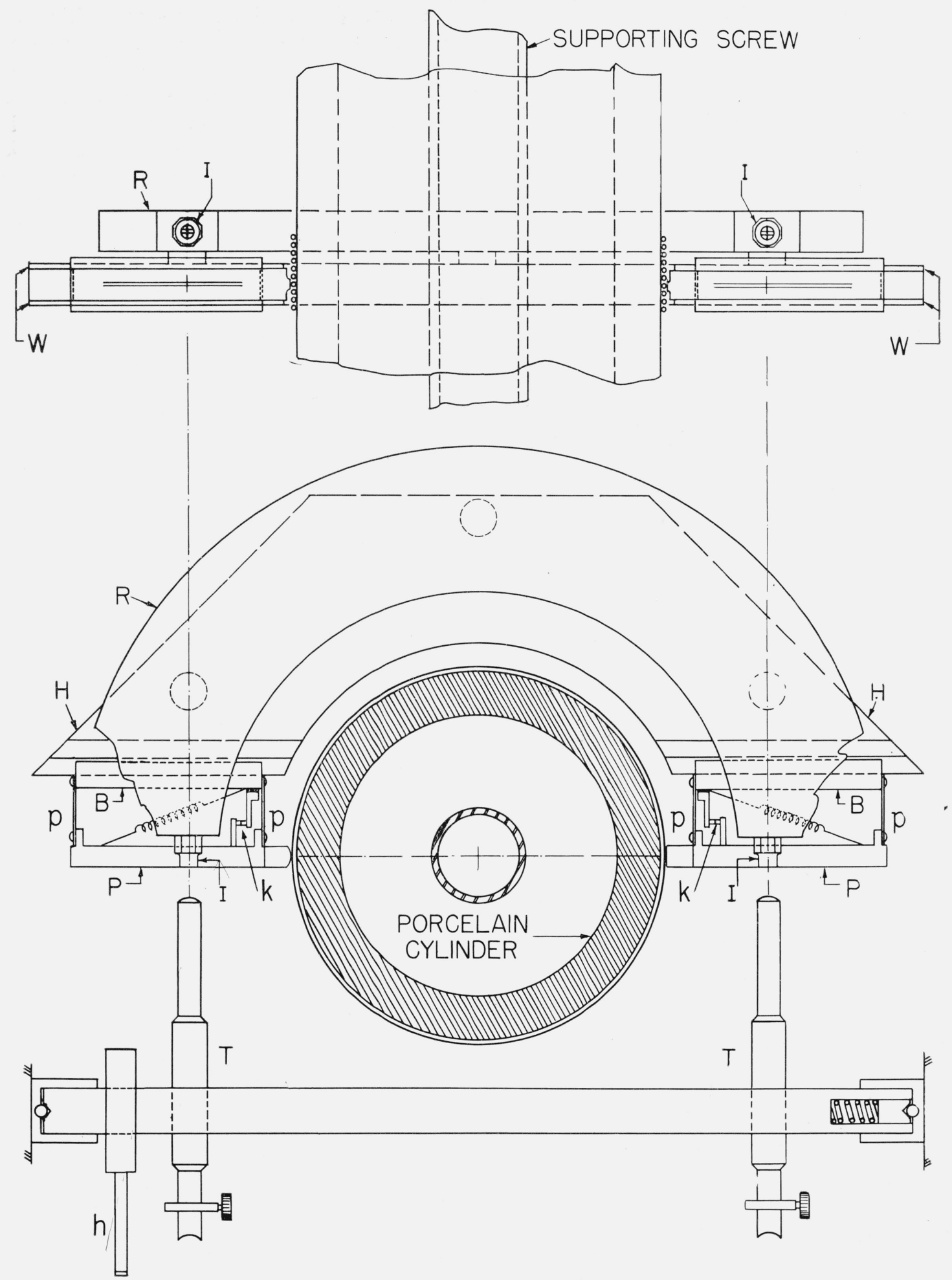

Figure 9. Simplified drawing to illustrate equipment used for primary-diameter measurements. 
(b) Diameter Measurements

After the primary of the mutual inductor is mounted and alined properly in the comparator, its diameter is measured with the apparatus shown in figure 9. A large steel "horseshoe", $H$, made of $35-\mathrm{mm}$ armor plate is mounted in a horizontal position. The two ends of the horseshoe are finished with well-alined ways, $W$, on which are hung the brass blocks, $B$. To these brass blocks, by means of thin rectangular strips of spring steel, $p$, are attached contact plates, $P$. By sliding the brass blocks toward the porcelain cylinder, the contact plates can be brought into contact with the wires of the primary, as shown. These contact plates carry fiducial marks, which can be observed through the telescopes, T. A set of fixed fiducial marks are on inserts, $I$, of polished steel set $50 \mathrm{~cm}$ apart in a second horseshoe, made of invar. This horseshoe, $R$, rests upon the smooth top surface of the first, $H$, at three places, and thin rubber pads between allow independent thermal expansion of the two horseshoes.

The procedure for making a diameter measurement is to slide the blocks, $B$, by means of handoperated screws until the plates, $P$, come in contact with the wire of the inductor. As soon as the contacts are made the contact plates will stop but the steel flexure plates will allow the blocks to continue to move, thus increasing the pressure on the contacts, but maintaining the alinement of the contact plates. As soon as the required pressure is attained, the breaker points, $k$, separate, extinguishing small electric lights, at which point the operator stops the motion of the blocks.

With the plates in contact with the wires of the helix, telescopes are sighted at the contact plates, and the cross-hairs of the micrometer eyepieces are set on the fiducial lines. The two telescopes, $T, T$, are mounted on a horizontal 5 -cm steel bar, and by means of the handle, $h$, they can be turned simultaneously. After the telescopes are set on the fiducial lines the bar is rotated slightly, tilting the telescopes, in order to bring the fixed reference marks on $I$ and $I$ into view. The micrometer eyepieces are then used to determine the difference in positions of the fixed and movable lines.

Figure 10 shows some of the details on the left side of the diameter-measuring micrometer. The letters identifying various parts correspond with those used in figure 9, with the exception of $S$, which is one of the hand-operated screws mentioned previously.

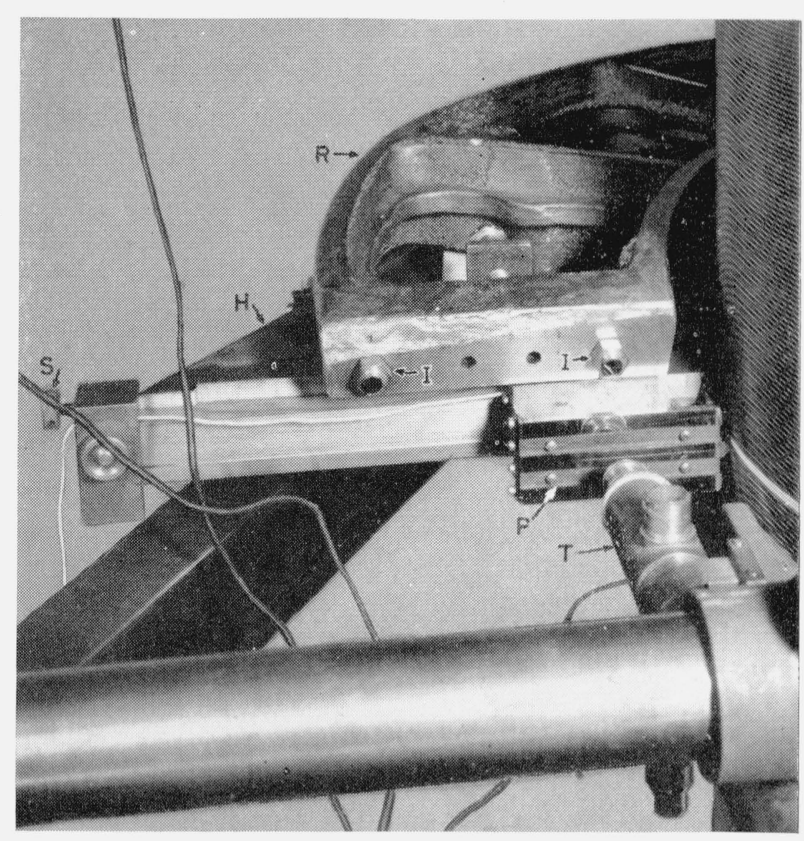

Figure 10. Left side of primary diameter-measuring micrometer.

It is realized that rotation of the bar carrying the telescopes will change their alinement because of the flexing of the bar. Hence at the end of a series of measurements, the cylinder and the movable contact plates are removed. A line standard is then placed at the level where the contact plates have been, and a transfer is made from the fixed reference lines to the lines on the calibrated meter bar. The two transfers eliminate the effect of the change of alinement of the telescopes and are the equivalent of a direct substitution of the standard meter bar.

After a series of measurements has been made on the cylinder, the two contact plates are brought to bear upon a calibrated gage wire on one side of the horseshoe, and a similar procedure is followed in measuring the distance between fiducial lines. As the diameter of the gage wire is accurately known, these readings give the amount that has to be subtracted from the measured distance between reference lines on the contact blocks.

The contact plates, $P$, are made of invar with a polished face on which are ruled the fiducial lines. These lines were ruled to match those of the standard meter bar by C. G. Peters of this Bureau. On one of the contact plates the surface that comes in contact with the wires of the inductor is slightly rounded with its axis parallel to the axis of the 
inductor, whereas on the other this surface is plane.

Before making a diameter measurement, the horseshoe is moved toward the cylinder until the readings are a maximum, indicating that the apparatus is set on a diameter. As the winding on the cylinder is a helix, the horseshoe is tilted until one contact surface is set lower than the other so that both center on wires of the helix. This slightly increases the apparent diameter of the helix, but a correction therefor is readily calculated.

The telescopes used for the measurements are of the auto-collimator type, and the micrometer eyepieces are the Geneva Society's type 0281. Divisions on the scales of the micrometers correspond to about a half micron in the fields of view of the telescopes. These scales were calibrated against tenth-millimeter graduations on the standard meter bar. Settings of the micrometer on lines of the meter bar or on fiducial marks usually can be repeated to one or two divisions on the micrometer scale, that is, to about a micron. As a large number of readings were made, this is considered to be sufficiently accurate for a determination of the average diameter of the primary to better than 1 part in a million.

Measurements of the diameter of the primary coil have consisted of a series of over 1,200 measurements, each turn of the helix being measured on three equally spaced diameters. At least once every five turns a transfer was made from the contact blocks to the fixed lines on the invar horseshoe. The air in the room where the measurements were made was kept in circulation by means of a large fan, and its temperature was thermostatically controlled at $23^{\circ} \mathrm{C}$. Frequent measurements of the temperature of the porcelain cylinder and of the steel horseshoe were made by means of attached thermocouples. These measurements were made by an observer in another room, who also recorded other data as they were transmitted by interphone.

Although only one observer remained in the room during the measurements, and the air was thermostatically controlled, the heat from the observer's body gradually raised the temperature of the comparator and of the porcelain cylinder. Fortunately the temperature changes were small and at a fairly uniform rate. After a period of 3 or $4 \mathrm{hr}$, it would be necessary to stop measurements and allow the temperature to fall. An effort was made to start not more than $0.1^{\circ} \mathrm{C}$ below $23^{\circ} \mathrm{C}$ and to take observations until the temperature rose to an equal amount above $23^{\circ} \mathrm{C}$ in order to avoid calculated corrections for temperature, as $23^{\circ} \mathrm{C}$ is the temperature to which all measurements are referred.

Measurements made in the manner outlined above give diameters to the outside surface of the wire. To obtain the diameter of the helix it is necessary to subtract the average wire diameter. The diameter of samples of the wire taken from the start, center, and finish of the primary winding was measured by means of an accurate micrometer caliper and found to be $1.6983 \mathrm{~mm}$ at $23^{\circ} \mathrm{C}$. The diameter at the beginning and at the end were the same to $\pm 0.0002 \mathrm{~mm}$, as was expected from the method of drawing. Using this value of the wire diameter, a preliminary value for the diameter of the primary helix to the center of the wires was obtained. From this diameter and the mass, number of turns and density of the wire, a more reliable value for the mean diameter of the wire on the primary winding was obtained. The mass and density were determined by the Metrology Division, although their determinations of density were for wire in the unstressed condition. The wire on the cylinder was wound under a tension of about $30 \mathrm{lb}$. From the stressstrain curve of a sample of the wire, the percentage elongation caused by this tension was calculated. Assuming a Poisson's ratio of 0.3 , the percentage change in diameter was calculated, and from the change in length and in cross section, the change in density was determined. This gave a density when stressed of $8.922 \mathrm{~g} / \mathrm{cm}^{3}$ at $23^{\circ} \mathrm{C}$ as against the measured value of $8.925 \mathrm{~g} / \mathrm{cm}^{3}$ for the unstressed wire. From the mass, density, and length the mean diameter, $2 r$, of the wire of the helix was calculated to be

$$
2 r=1.6986 \mathrm{~mm}
$$

at $23^{\circ} \mathrm{C}$.

A complete series of diameter measurements was made in December 1947, and repeated in June 1948, with minor changes in the measuring equipment. From the December 1947, observations a value of $40.72740 \mathrm{~cm}$ was obtained for the mean horizontal diameter of the helix to the centers of the wire. From the June 1948, observations a value of $40.72754 \mathrm{~cm}$ was obtained. Data obtained from the latter observations were used in preparing figure 11 . The curves labeled 


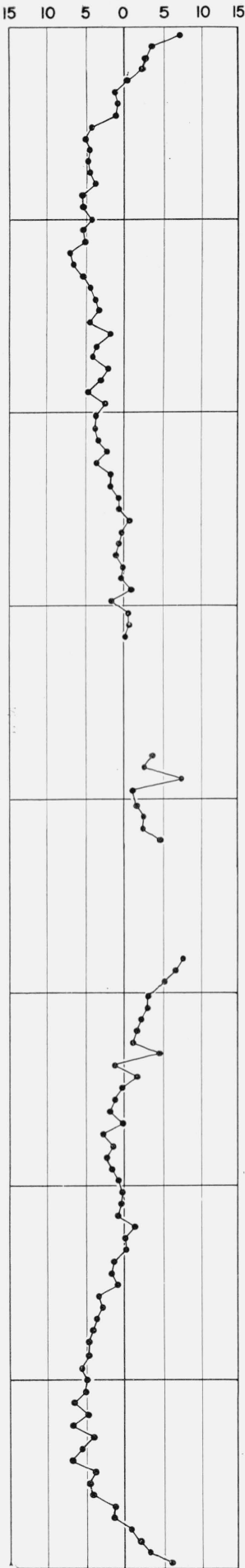

$0^{\circ}$

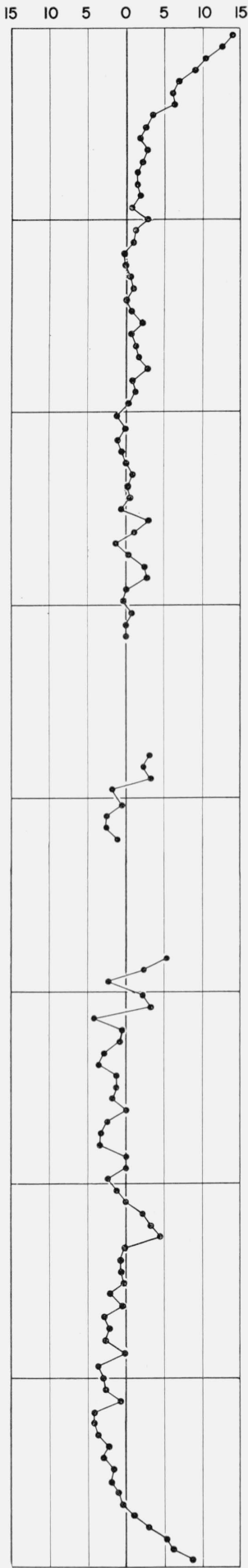

$120^{\circ}$

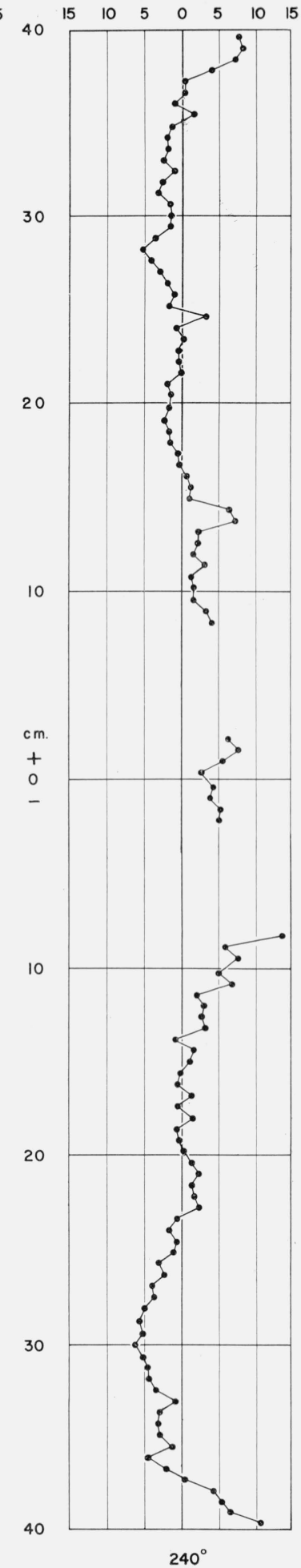

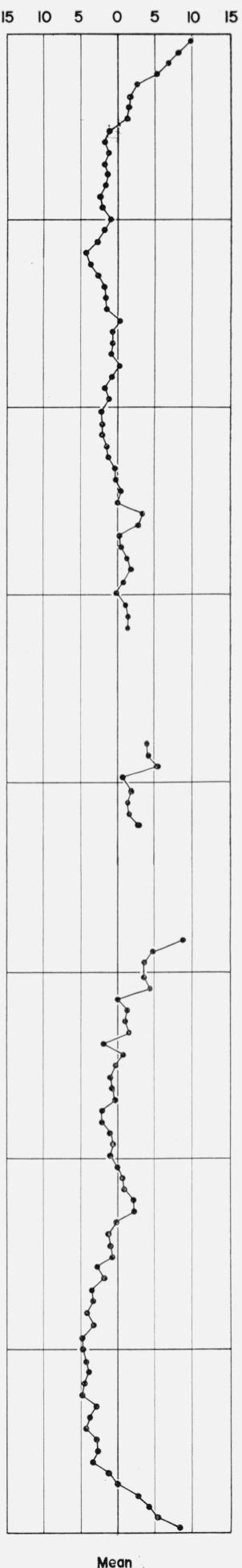

FIgURE 11. Variation in primary diameter based upon June 1948 measurements. Zero $=$ mean horizontal diameter to center of wire $=40.7275 \mathrm{~cm}$. 
$0^{\circ}, 120^{\circ}$, and $240^{\circ}$ show diameter variations in microns along three pairs of elements $120^{\circ}$ apart, whereas the curve on the right is the mean of the other three. The curves are plotted with respect to the mean diameter of the 343 turns that constitute the active portion of the primary winding; the mean diameter values given above also pertain to these turns. For the long 159turn sections of the primary, each plotted point is the mean of three turns, whereas for the short 25-turn middle section, each point represents the mean of five turns. In table 1, mean diameter deviations in microns with respect to the grand mean are shown for each of the three sections of the coil in each of the three planes. The mean given at the foot of each column is obtained by weighting the figures for each section according to the number of turns involved.

TABLE 1. Diameter deviations, microns

\begin{tabular}{|c|c|c|c|c|}
\hline & $0^{\circ}$ & $120^{\circ}$ & $240^{\circ}$ & Mean \\
\hline Top: 159 turns & -2.3 & +2.1 & +0.3 & 0.0 \\
\hline Middle: 25 turns .... & +3.3 & -0.2 & +4.9 & +2.7 \\
\hline Bottom: 159 turns ... & -1.1 & -.5 & +0.3 & -0.4 \\
\hline Weighted mean & -1.3 & +.7 & +.6 & .0 \\
\hline
\end{tabular}

Giving equal weight to the December 1947 and June 1948 observations, the mean radius of the primary helix at $23.0^{\circ} \mathrm{C}$, measured to the center of the wire, was

\section{$a_{0}=20.36373 \mathrm{~cm}$,}

as of the mean date March 1948. The apparent uncertainty in $a_{0}$ is approximately $0.4 \mu$, or 2 $\mathrm{ppm}$, and this in turn is equivalent to about $4 \mathrm{ppm}$ in terms of inductance.

\section{(c) Pitch Measurements}

The porcelain cylinder was wound with $1.7-\mathrm{mm}$ copper wire, and as the winding pitch is $2 \mathrm{~mm}$, the clearance between wires is $0.3 \mathrm{~mm}$. To measure the pitch, measurements were made from centers of the clearance between wires, instead of being made between lines drawn on the wires or from the estimated centers of the wires.

To locate the centers between wires, two 4 -mm steel rods were ground to a point by using a taper of about $37.5^{\circ}$. When mounted radially and pressed lightly between two wires, the centers of these sharpened rods or "pins," line up with the centers of the spaces between the wires. About a centimeter back from the points the pins were ground half way through to give flat surfaces at the axes. These surfaces were polished and marked with fiducial lines that were made approximately parallel to the axes of the pins.

In order to use this method of measuring pitch it is necessary to make sure that the pins are lined up with the radii of the porcelain cylinder unless this alinement is the same for all measurements. To obtain this result the contact pins are attached to frameworks that move back and forth in the direction of a radius of the cylinder on ways that are supported by a heavy vertical rod.

The construction of one of the frameworks that is used in carrying the contact pin is shown schematically in figure 12 . The pin, $P$, is at-

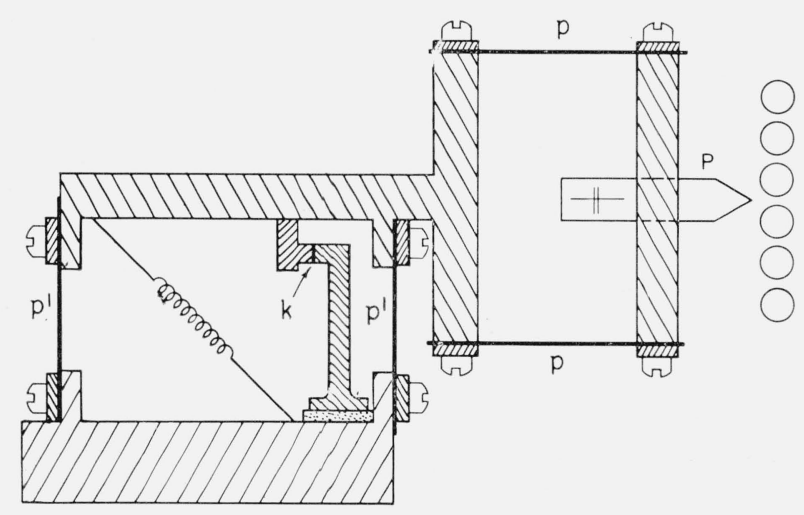

Figure 12. Support for pitch-measuring pin.

tached to a rectangular brass piece about $3 \mathrm{~mm}$ thick, part of which has been removed to decrease the weight. This rectangular piece is attached to the remainder of the framework by means of two thin rectangular strips of spring steel $p, p$. These strips are about 2 by $4 \mathrm{~cm}$ and $0.07 \mathrm{~mm}$ thick. As the steel pin is pushed between two wires of the cylinder, the steel "flexure plates" bend and allow the point to set itself in the center of the blank space between two wires. This motion takes place in such a way that the fiducial line moves up or down, keeping the line always parallel to its original direction. To prevent damage to the wires, this part of the framework is attached to its base by means of a second set of flexure plates $p^{\prime} p^{\prime}$, set at right angles to the first pair, as shown. These plates can also bend without changing the direction of the fiducial line. The base carrying this framework is moved along the ways by means 
of a coarse screw until the plates $p^{\prime} p^{\prime}$ bend and the contact at $k$ opens. The two parts of this contact are electrically insulated and connected into a circuit with a small lamp and a battery. The tension in the coiled spring that is shown keeps the contact normally closed. If the forward motion in the ways is stopped as soon as the lamp goes off, showing that $k$ has been opened, practically the same force is applied each time to the wires of the inductor. This force is kept low so that no permanent deformation of the wires is produced. The location of the fiducial line does not change with reasonable changes of the force, and a setting can be made quickly and with little care on the part of an observer.

Figure 13 shows how the framework of figure 12

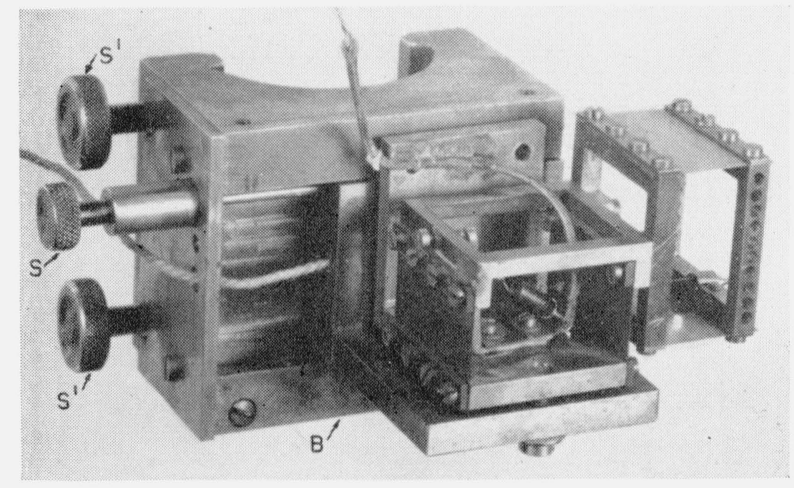

Figure 13. Pitch-measuring pin assembly.

is mounted on a base that can be moved along guide ways in the block, $B$, by means of a handoperated screw, $S$. By means of the screws, $S^{\prime} S^{\prime}$, the whole assembly can be clamped rigidly at any desired height on a heavy vertical sieel mast.

To measure the distance between two fiducial lines, two telescopes are mounted on a vertical shaft and focused on the lines. These telescopes have micrometer eyepieces, the lines of which are set on the images of the fiducial lines. The shaft carrying the telescopes is then rotated to bring into view lines on a calibrated meter bar. The changes in the readings of the micrometer eyepieces when reset to images of lines on the meter bar give a measurement of the distance between contact pins. After a set of such readings the frameworks carrying the contact pins are interchanged, and the readings are repeated. This interchange eliminates any error from the fiducial lines' not being exactly on the axes of the contact pins or from their inexact alinement. From the average of the two sets of measurements, data are obtained for calculating the average pitch of the winding in the section between the contact pins.

Figure 14 is a closeup view of the complete pitch-measuring equipment, with the calibrated meter bar at $M$. The telescopes shown were used only in preliminary work. They were later replaced with more rigid assemblies firmly held in $V$-grooves in stiff castings attached to the telescope mast.

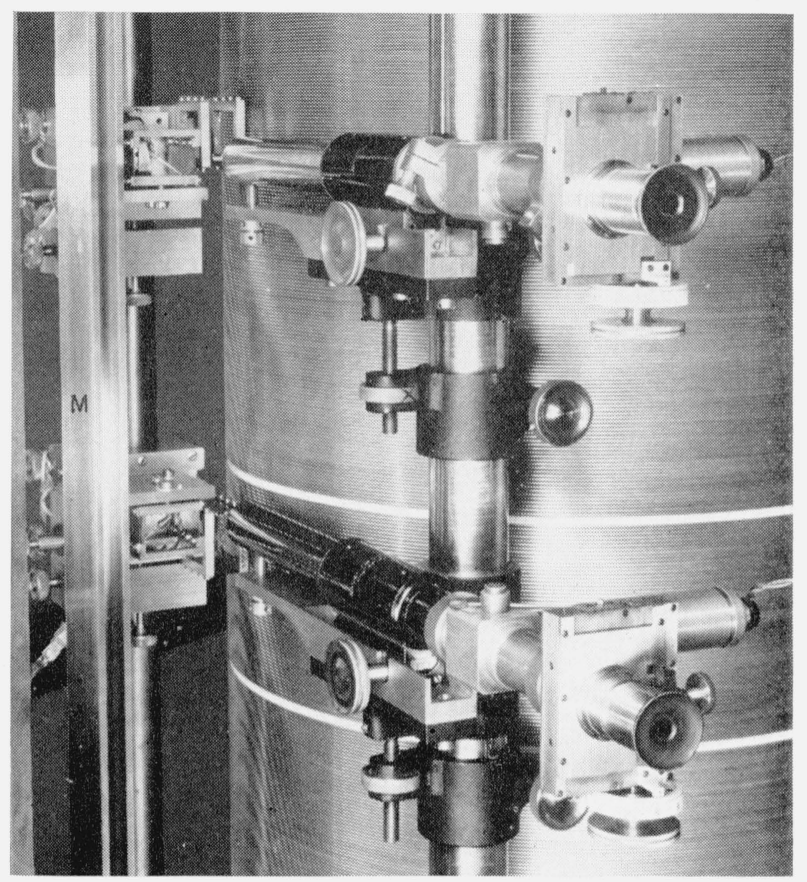

Figure 14. Close-up view of pitch-measuring equipment.

The measurement of the pitch of the primary winding was carried out in two steps. An average pitch was first determined by measurements of the distance between turns in a group near one end and corresponding turns in a group near the other end. Using this average pitch, the locations of the intermediate turns were computed, assuming the pitch to be uniform. Measurements were then made to determine the deviations in location of these intermediate turns from their computed positions. These small deviations in axial location of turns are employed later in calculating a correction to the value of mutual inductance due to pitch irregularities. At this point it should perhaps be emphasized that the accurately lapped groove 
in which the wire is wound is one continuous helix throughout the length of the cylinder, and that therefore the distance, $s$, between any two wires or grooves in any section of the winding along a given element of the cylinder is an integral number times the pitch. The nominal value of this distance, $s_{n}$, is $0.2 n \mathrm{~cm}$, where $n$ is the number of turns in the interval.

More accurately expressed,

$$
s=n p_{0}+c .
$$

The two steps involved in the pitch measurements, therefore, are the determination of the average pitch, $p_{0}$, and the evaluation of the corrections, $c$, which are a measure of the axial mislocation of turns.

For determination of the average pitch the contact pins were set to measure an interval of $70 \mathrm{~cm}$ (350 turns). Starting with one pin at the top end of the primary winding a series of readings was taken as the cylinder was raised. Readings were taken every one-third turn until the lower end of the winding was raised to the position of the bottom pin. This gave readings from the top 50 to the bottom 50 turns, and the average of the 150 determinations gave the length for 350 turns. The average pitch as above defined was measured three times during the spring of 1948 with the following results:

\begin{tabular}{|c|c|}
\hline 1948 & $\begin{array}{c}p_{0} \text { at } 23.0^{\circ} \mathrm{C}, \\
\text { in } \mathrm{cm}\end{array}$ \\
\cline { 1 - 2 } & \\
\hline February & 0.1999935 \\
April & 1999939 \\
May & 1999940 \\
\hline
\end{tabular}

From these data the average value of $p_{0}$ at $23.0^{\circ}$ C was taken to be

$$
p_{0}=0.1999938 \mathrm{~cm},
$$

as of March 1948. The apparent uncertainty in $p_{0}$ was about $1 \mathrm{ppm}$, and the corresponding uncertainty in mutual inductance is likewise about $1 \mathrm{ppm}$. This value of $p_{0}$ will be used later in the calculation of mutual inductance.

The evaluation of the corrections associated with pitch imperfections was somewhat complicated by the lack of a perfect turn to which to refer the others. Instead of using one turn, or the groove between two adjacent turns, as a reference, it was decided to refer all pitch measurements to the central 10 turns of the groove symmetrically located with respect to the plane $x=0$. Preliminary measurements between these and other groups located at various distances from the central plane showed that the pitch correction would be less than $5 \mathrm{ppm}$. These preliminary measurements were made considering each of the long top and bottom sections of the primary winding to be divided into eight groups of 20 turns each, as indicated in figure 15 .

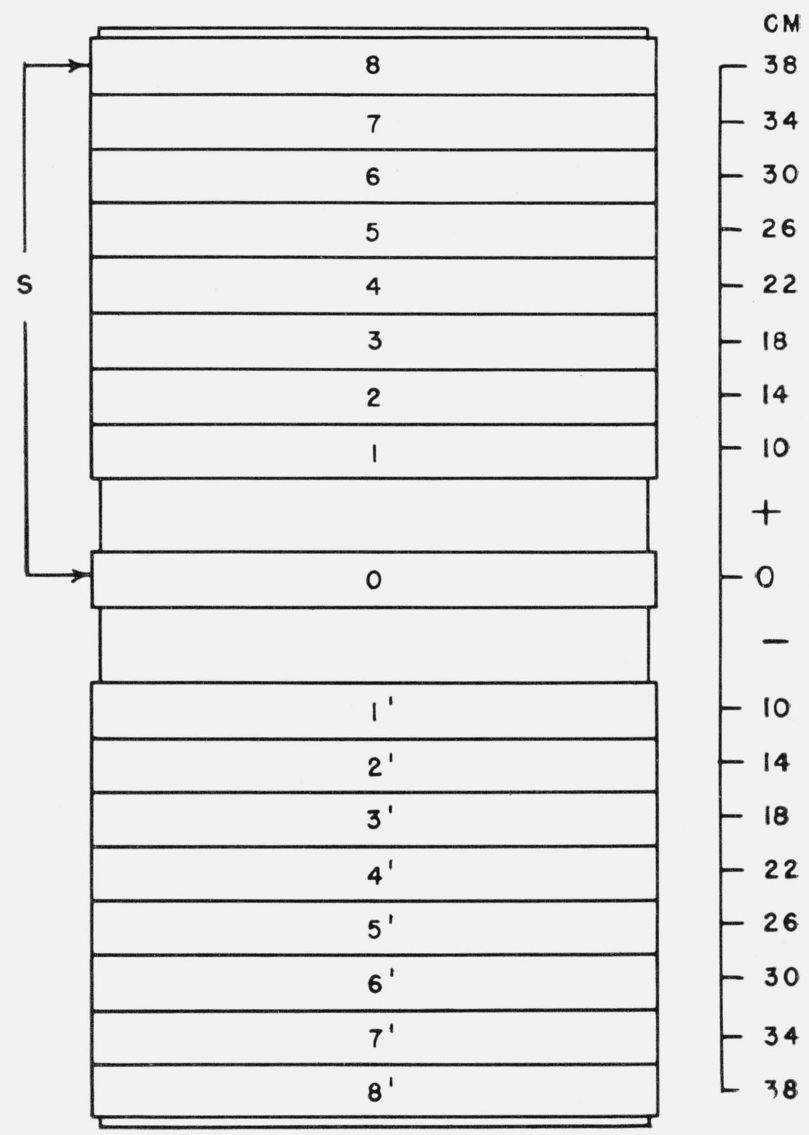

FIGURE 15. Relative location of various groups of primary turns.

In each of these 16 sections the space between the middle 10 turns of wire formed the groove that was referred to the central 10-turn groove. The nominal distance between the center of the central reference group and the center of group 8, for example, was $38 \mathrm{~cm}$. The pins of the pitch measuring apparatus were set with this nominal 
spacing and the cylinder adjusted in height until the bottom pin coincided with the top of the central 10-turn groove. The interval, $s$, was then measured six times per revolution as the cylinder was raised 10 turns, thus giving 60 measurements of the distance, $s$, the average of which was considered to define the mean location of all the turns in group 8. From similar measurements made upon the remaining 15 groups, data were obtained that enabled the pitch correction to be calculated. The method outlined above could not be carried out with high accuracy, because the $10-\mathrm{cm}$ distance 0 to 1 and 0 to $1^{\prime}$ could not be measured directly, as the distance between the measuring pins could not be reduced below $15 \mathrm{~cm}$. The method described below, involving only half as many observations, was found to be better suited to our needs.

Since the pitch corrections are small, and since a high degree of symmetry exists in the primary winding, it is sufficient to measure the average distance between symmetrically located groups, such as 8 and 8 , instead of between each group and the central group. If, for example, the spacing of groups 8 to $8^{\prime}$ were $5 \mu$ too high, the total correction due to the axial mislocation of groups 8 and $8^{\prime}$ would be the same no matter how the $5-\mu$ error was apportioned between them. Accordingly, the final pitch measurements were made by determining the average distance, $\bar{s}$, between the eight pairs of symmetrically located groups of 20 turns. In this series of observations the distance, $s$, was measured three times per revolution as the cylinder was raised or lowered 20 turns, thus giving 60 determinations distributed over the whole 20 turns. The general procedure was as follows: the measuring pins were spaced $76 \mathrm{~cm}$ apart and the cylinder height adjusted until the pins were opposite the top ends of the grooves in sections 8 and $8^{\prime}$. The cylinder was then raised 20 turns, the distance $s$ being measured at the required settings. The blocks supporting the measuring pins were reversed in position at the completion of each odd-numbered turn, and readings on the invar line standard were taken periodically. The spacing of the measuring pins was then reduced to $68 \mathrm{~cm}$ and the pins so located that they were opposite the bottom ends of the grooves in sections 7 and $7^{\prime}$. The cylinder was then lowered 20 turns, while measuring $s$ and reversing the blocks in the established manner.
This procedure was continued until $\bar{s}$ had been determined for all eight intervals. The results are presented in table 2. Here $n$ is the number of turns in the interval; $s_{n}$ is the nominal spacing of the groups and equals $0.2 n ; s_{0}$ is the spacing as calculated from the mean pitch, and equals $n p_{0} ; \bar{s}$ is the average of 60 measurements of $s$ for each interval; $\bar{c}$ equals $\bar{s}-s_{0}$. The quantities $\bar{c}$ will be used for calculating the pitch correction in a later section of this paper.

Table 2. Pitch-variation data

\begin{tabular}{|c|c|c|c|c|c|}
\hline Interval & $n$ & $s_{n}$ & $S_{0}$ & $\bar{s}$ & $\bar{c}$ \\
\hline & & $\mathrm{cm}$ & $\mathrm{cm}$ & $\mathrm{cm}$ & $\mu$ \\
\hline 8 to $8^{\prime} \ldots$ & 380 & 76 & 75.99764 & 75.99760 & -0.4 \\
\hline 7 to $7^{\prime} \ldots$ & 240 & 68 & 67.99789 & 67. 99794 & +.5 \\
\hline 6 to $6^{\prime} \ldots$ & 300 & 60 & 59. 99814 & 59. 99788 & -2.6 \\
\hline 5 to $5^{\prime} \ldots$ & 260 & 52 & 51. 99839 & 51. 99801 & -3.8 \\
\hline 4 to $4^{\prime} \ldots$ & 220 & 44 & 43. 99864 & 43. 99866 & +.2 \\
\hline 3 to $3^{\prime} \ldots$ & 180 & 36 & 35. 99888 & 35.99896 & +.8 \\
\hline 2 to $2^{\prime} \ldots$ & 140 & 28 & 27. 99913 & 27. 99897 & -1.6 \\
\hline 1 to $1^{\prime} \ldots$ & 100 & 20 & 19. 99938 & 19. 99918 & -2.0 \\
\hline
\end{tabular}

It will be noted that the quantities $\bar{c}$ have been determined by taking the difference between two large quantities and are therefore apparently subject to appreciable error. However, the $\bar{c}$ values need be known only approximately, inasmuch as they will be used later to calculate a correction that is small. Otherwise expressed, the care with which temperatures need be controlled when measuring $\bar{s}$ is neither more nor less than that required in making any pitch measurement. The lengths given in table 2 pertain to a temperature of $23.0^{\circ} \mathrm{C}$.

It may be remarked that the last-described measurements are the necessary and sufficient observations as far as pitch is concerned. The average pitch, $p_{0}$, determined from observations over the $70-\mathrm{cm}$ interval need not necessarily be measured, inasmuch as any arbitrary value may be assumed and deviations $\bar{c}$ calculated with reference to the $s_{0}$ levels so established. Alternatively, $p_{0}$ could be taken as the mean of eight pitch values obtained from table 2 by dividing each value of $\bar{s}$ by the corresponding number of turns, $n$. The average pitch as determined from observations over the $70-\mathrm{cm}$ interval is of some historical interest, however, because this interval was measured in 1938 and again in 1948. The observations involved can be made in a day, when the equipment has been set up, and the results 
furnish information regarding the change in overall length over long periods of time.

\section{(d) Straightness Measurements}

The formulas available for calculating mutual inductance are usually based upon the assumption that all turns are coaxial. Chester Snow, of this Bureau, has provided a formula that will be employed in a later section of this paper to calculate the correction for sidewise displacement, or eccentricity, of turns in the inductor. In order to apply this formula, some rough measurements were made to determine the straightness of the primary winding. A special measuring pin, illustrated in figure 16 , was made and mounted in one

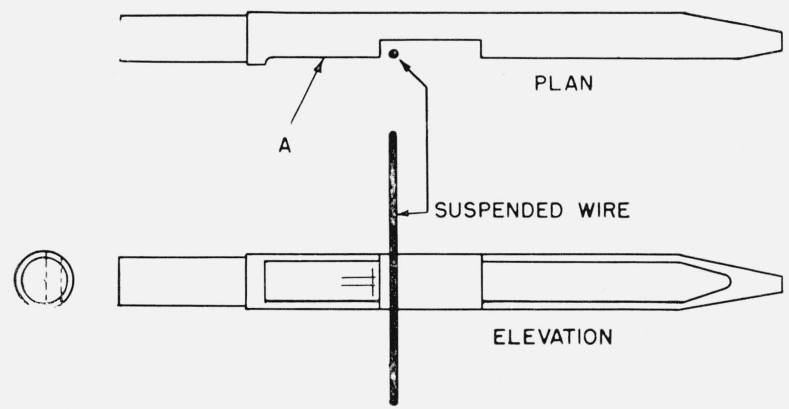

Figure 16. Measuring pin used in straightness measurements.

of the pitch-measuring blocks. This pin was used to measure the distance from the surface of the winding to a fine wire suspended vertically in the slot in the measuring pin. One of the telescopes used for the pitch measurements was employed to observe the distance between the center of the suspended wire and a fiducial line ruled on the polished surface, $A$, of the pin. The location of the fiducial line and the proportions of the apparatus were such that the wire and the fiducial line focused simultaneously in the field of view of the telescope. Observations were made along a given element of the cylinder by setting the measuring pin and telescope at various $x$-distances. The cylinder was left stationary. The observations furnished data from which the displacement of turns in the top and bottom portions of the cylinder could be calculated with respect to the central 25 turns of the primary winding. From observations made along four elements in two planes at right angles, it was concluded that no portion of the top and bottom sections of the primary was off-center by more than $10 \mu$ with respect to the axis of the central 25 turns of the primary. In these measurements no attempt was made to work closer than about $\pm 5 \mu$. Some slight difficulty was experienced at first before the proper technique was evolved for obtaining a suspended fine wire with the requisite straightness.

\section{Measurements of the Secondary}

The multilayer secondary coil was mounted in a glass channel with transparent sides in order that the diameters of the layers could be determined after the winding was completed. This eliminated the uncertainty as to the amount the inner layers were compressed by the outside layers, which would arise if the diameter had been determined for each layer as it was wound.

For determinations of the diameters of the layers, use was made of a special comparator. This comparator was set to measure diameters, and readings were made to $0.01 \mathrm{~mm}$ on each layer. The diameters were determined at four equally spaced positions around the coil. The coil was then inverted and the corresponding readings taken for the turns at the other end of each layer.

Four measurements from each face of the secondary gave eight values that were averaged to obtain the diameter of a layer. The complete set of measurements was made by two separate observers, and no difference in mean diameter obtained by the two observers exceeded $0.05 \mathrm{~mm}$. The dimensions were determined at $23^{\circ} \mathrm{C}$., which is the temperature at which the inductor is preferably used.

The values of the average radii of the $24 \mathrm{com}$ plete layers and of the partial twenty-fifth layer are given in table 3 . In this table are also given the radii that would have been obtained if the layers had been uniformly spaced between the first and last. This shows how the inner layers were displaced because of compression, and the column marked "Compression" shows the errors in dimensions that would have resulted if the inner and outer radii had been determined and the other radii had been calculated on the assumption of uniform spacing. The mean radius, $A_{0}$, to the center of the secondary channel is taken as the mean radius of layers 1 and 24 . Therefore, at a temperature of $23^{\circ} \mathrm{C}$,

$$
A_{0}=26.348 \mathrm{~cm} \text {. }
$$


TABLE 3. Radii of secondary layers of mutual inductor

\begin{tabular}{|c|c|c|c|}
\hline Layer & Average radius & $\begin{array}{l}\text { Uniform dis- } \\
\text { tribution }\end{array}$ & $\begin{array}{l}\text { Compres- } \\
\text { sion }\end{array}$ \\
\hline & $\mathrm{mm}$ & $m m$ & $\mathrm{~mm}$ \\
\hline 1 & 251. 28 & 251.28 & 0.00 \\
\hline 2 & 252.32 & 252.34 & .02 \\
\hline 3 & 253.38 & 253.40 & .02 \\
\hline 4 & 254.43 & 254.47 & .04 \\
\hline 5 & 255.49 & 255.53 & .04 \\
\hline 6 & 256.54 & 256.59 & .05 \\
\hline 7 & 257.60 & 257.65 & .05 \\
\hline 8 & 258.65 & 258. 71 & .06 \\
\hline 9 & 259. 71 & 259. 78 & .07 \\
\hline 10 & 260.75 & 260.84 & .09 \\
\hline 11 & 261.82 & 261.90 & .08 \\
\hline 12 & 262.87 & 262. 96 & .09 \\
\hline 13 & 263.95 & 264.02 & .07 \\
\hline 14 & 265.01 & 265.09 & .08 \\
\hline 15 & 266. 09 & 266.15 & .06 \\
\hline 16 & 267.15 & 267. 21 & .06 \\
\hline 17 & 268. 22 & 268. 27 & .05 \\
\hline 18 & 269. 28 & 269.34 & .06 \\
\hline 19 & 270.33 & 270.40 & .07 \\
\hline 20 & 271.40 & 271.46 & .06 \\
\hline 21 & 272.47 & 272.52 & .05 \\
\hline 22 & 273.53 & 273.58 & .05 \\
\hline 23 & 274.58 & 274.65 & .07 \\
\hline 24 & 275.68 & 275. 71 & .03 \\
\hline 25 & 276.77 & 276.77 & .00 \\
\hline
\end{tabular}

\section{Calculation of Mutual Inductance}

\section{The Idealized Inductor}

(a) General Considerations

The computation of mutual inductance is approached by first considering the idealized inductor represented by figure 17 . The basic dimensions given in this figure have been assembled from section IV of this paper and pertain to the 1948 series of dimensional measurements. The secondary is taken as a circle of radius $A_{0}$ located at the center of the secondary channel. The mutual inductance between a helix of axial length $x$ and a coaxial circle located in the plane of one end of the helix is given by the well-known Jones formula [8], which is here expressed in the form

$$
M=2 \pi N_{x}(A+a) c k\left\{\frac{\mathrm{K}-\mathrm{E}}{k^{2}}-\frac{c^{\prime 2}}{c^{2}}(\mathrm{I}-\mathrm{K})\right\} .
$$

In this equation, $N_{x}$ is the number of primary turns in the axial distance 2 , and $\mathrm{K}, \mathrm{E}$, and $\Pi$ are the complete elliptic integrals of the first, second, and third kind, respectively, to the modulus $k$

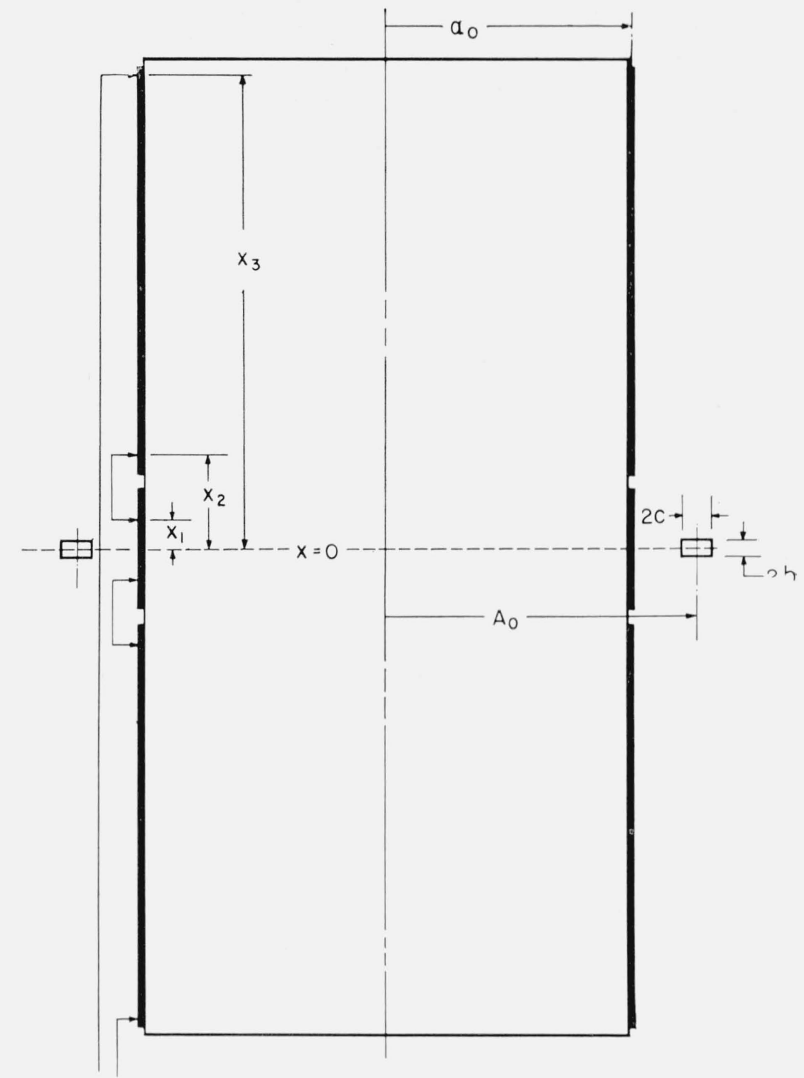

\begin{tabular}{|r|}
\hline 1948 DIMENSIONS, $\mathrm{cm}$ \\
\hline$a_{0}=20.36373$ \\
$A_{0}=26.348$ \\
$P_{0}=0.1999938$ \\
$x_{1}=12.5 p_{0}=2.49992$ \\
$x_{2}=40.5 p_{0}=8.09975$ \\
$x_{3}=199.5 p_{0}=39.89876$ \\
$2 c=2.546$ \\
$2 b=0.82$ \\
\end{tabular}

FIGURE 17. Idealized inductor with 1948 values for principal dimensions.

and parameter $c$, where

$k^{2}=\frac{4 A a}{(A+a)^{2}+x^{2}}, c^{2}=\frac{4 A a}{(A+a)^{2}}, c^{\prime 2} \equiv 1-c^{2}=\frac{(A-a)^{2}}{(A+a)^{2}}$.

The mutual inductance, $M_{0}$, of the idealized inductor is given by the expression

$$
M_{0}=2\left(M_{1}-M_{2}+M_{3}\right),
$$

where $M_{1}, M_{2}$, and $M_{3}$ are obtained from eq. 5 by using for $x$ the corresponding values of $x_{1}, x_{2}$, and $x_{3}$, and the factor 2 accounts for the other half of the primary, which is symmetrically located with respect to the plane $x=0$. 
During the early portion of this investigation the methods of L. V. King [9] were used extensively for evaluating the elliptic integrals involved in the above equations. Shortly before the war there appeared an article by Bartky [10] on the numerical calculation of a generalized complete elliptic integral. By using his methods it became possible to evaluate the complete elliptic integral of the third kind without recourse to tabulated functions of any kind. Bartky's method has proved well suited to the machine calculation of all three types of elliptic integrals involved in the Jones equation. Inasmuch as King's methods are now fairly well known, the material that follows will devote attention mainly to the Bartky method, although a combination of both methods is favored and an illustration of this combined procedure will be given.

In executing machine calculations of the type described in the sections to follow, the work has habitually been carried to eight figures in order that there should be no uncertainty exceeding one in the seventh place arising out of the purely mechanical features of computation, there being no implication that such accuracy is warranted by the basic data.

(b) Evaluation of Ellipitic Integrals

Bartky's formula 23 was found to be unsuitable because of unsatisfactory convergence of the function $F_{i}\left(R_{i}\right)$ in the region of interest in this work. His formula 36, however, was found to be quite satisfactory. Employing several obvious changes in notation it is written here in the form

$$
\begin{aligned}
I & =\int_{0}^{\frac{\pi}{2}} \frac{\alpha \zeta \cos ^{2} \phi+\beta \delta \epsilon \sin ^{2} \phi}{\alpha \cos ^{2} \phi+\beta \delta \sin ^{2} \phi} \frac{\partial \phi}{R} \\
& \equiv \int_{0}^{\frac{\pi}{2}} F(R) \frac{\partial \phi}{R}
\end{aligned}
$$

where $R^{2}=\alpha^{2} \cos ^{2} \phi+\beta^{2} \sin ^{2} \phi$, and $\alpha, \beta, \delta, \epsilon$, and $\zeta$ are constants. By repeated application of Landen's transformation to eq 9, using the recurrence formulas

$$
\begin{aligned}
\alpha_{i+1} & =\frac{1}{2}\left(\alpha_{i}+\beta_{i}\right), \quad \beta_{i+1}=\left(\alpha_{i} \beta_{i}\right)^{3 / 2} \\
\delta_{i+1} & =\frac{\beta_{i+1}}{4 \alpha_{i+1}}\left(2+\delta_{i}+\delta_{i}^{-1}\right), \epsilon_{i+1}=\frac{\delta_{i} \epsilon_{i}+\zeta_{i}}{1+-\delta_{i}} \\
\zeta_{i+1} & =\frac{1}{2}\left(\epsilon_{i}+\zeta_{i}\right),
\end{aligned}
$$

it is found that $\alpha$ and $\beta$ approach a common limit $\alpha_{\infty} ; \delta$ approaches the limit 1 , while $\epsilon$ and $\zeta$ approach a common limit $\zeta_{\infty}$. In the limit, the integral (eq 8) is given by

$$
I=\frac{\pi}{2}\left(\frac{\zeta_{\infty}}{\alpha_{\infty}}\right)
$$

By a proper choice of values for the five constants appearing in eq 8 , the integral $I$ becomes $\mathrm{K}$, E, II, or combinations of these three elliptic integrals. For example, if $k^{\prime}=\sqrt{1-k^{2}}$ and the constants are so chosen that $\alpha=1, \beta=k^{\prime}, \delta=1 / k^{\prime}$, $\epsilon=1$, and $\zeta=1$ the integral (eq 8) becomes

$$
I=\int_{0}^{\frac{\pi}{2}}\left(1-k^{2} \sin ^{2} \phi\right)^{-1 / 2} \partial \phi,
$$

which is recognized as the complete elliptic integral of the first kind, K. In applying Landen's transformation to the evaluation of eq 12 the numerical values of the five constants as chosen above become the initial values in the recurrence eq 10 , corresponding to $i=0$. It will be noted that for the $\mathrm{K}$ integral all terms of the $\epsilon$ and $\zeta$ scales equal unity, and eq 11 becomes

$$
\mathrm{K}=\frac{\pi}{2}\left(\frac{1}{\alpha_{\infty}}\right) \text {. }
$$

Those familiar with King's methods will recognize the similarity between his eq 25 and our eq 13 where $\alpha_{\infty}$ is the end term in the arithmeticogeometrical scale defined by the $\alpha$ and $\beta$ scales,

\begin{tabular}{|c|c|c|c|c|c|}
\hline$I$ & $\alpha$ & $\beta$ & $\delta$ & $\epsilon$ & $\zeta$ \\
\hline $\mathrm{K}$ & 1 & $k^{\prime}$ & $\frac{1}{k^{\prime}}$ & 1 & 1 \\
\hline E & 1 & $k^{\prime}$ & $\frac{1}{k^{\prime}}$ & $k^{\prime 2}$ & 1 \\
\hline$\Pi$ & 1 & $k^{\prime}$ & $\frac{c^{\prime 2}}{k^{\prime}}$ & $\frac{1}{c^{\prime 2}}$ & 1 \\
\hline$(\mathrm{K}-\mathrm{E})$ & 1 & $k^{\prime}$ & $\frac{1}{k^{\prime}}$ & $k^{2}$ & 0 \\
\hline$(\Pi-\mathrm{K}$ & 1 & $k^{\prime}$ & $\frac{c^{\prime 2}}{k^{\prime}}$ & $\frac{c^{2}}{c^{\prime 2}}$ & 0 \\
\hline
\end{tabular}
which correspond to King's $a$ and $b$ scales.

In table 4 there have been tabulated the values that should be used for the five constants in order that eq 8 should become the integral listed in the left-hand column.

TABLE 4. Values of the arbitrary constants that transform the generalized integral to those listed under I 
Three examples of the application of these methods to the computation of various elliptic integrals will now be given. In the first example, presented in table $5, \mathrm{~K}, \mathrm{E}$, and $\mathrm{II}$ have been computed separately and directly for the case corresponding to $x=x_{1}$ on the idealized inductor of figure 17. For each integral $\mathrm{K}, \mathrm{E}$, and $\Pi$ the values of $\alpha, \beta, \delta, \epsilon$, and $\zeta$ are to be taken as indicated in table 4 . As stated above, these values of $\alpha, \beta, \delta, \epsilon$, and $\zeta$ then become the initial values in the scales (eq 10) corresponding to $i=0$. Their numerical values for a specific case are obtained from the dimensions of the inductor by using eq 6 and the relation

$$
k^{\prime 2} \equiv 1-k^{2}=\frac{(A-a)^{2}+x^{2}}{(A+a)^{2}+x^{2}}
$$

In connection with the evaluation of the K integral, it should be noted that the $\delta$ scale has a definite series of values, but in this particular case they are without effect on the value of $K$ and are therefore not required and are not tabulated in any of the illustrative examples. The $\delta$ scale is required, however, in connection with the evaluation of both the $\mathrm{E}$ and the $\Pi$ integrals. It should be further noted that $\alpha$ and $\beta$ are the same for all integrals listed in table 4 , and that therefore the $\alpha$ and $\beta$ scales need be evaluated only once when calculat. ing $\mathrm{K}, \mathrm{E}$, and $\Pi$ for a given set of $A, a$, and $x$ values. A study of the above will reveal that the values assembled in table 5 are the necessary and sufficient data required for calculating $\mathrm{K}, \mathrm{E}$, and $\Pi$ for the given dimensional conditions. In the column following the $\beta$ scale it has been found convenient to tabulate the factor $\eta_{i} \equiv \beta_{i} / 4 \alpha_{i}$ which is required in calculating the $\delta$ scales.

TABle 5. Computation of integrals $K, E$, and $I I$ for $x=x_{1}=2.49992 \mathrm{~cm}$

$k^{\prime 2}=0.019221464 \quad \quad k^{\prime}=0.13864149 \quad \frac{1}{k^{\prime}}=7.2128480 \quad \frac{c^{\prime 2}}{k^{\prime}}=0.11837970 \quad \frac{1}{c^{\prime 2}}=60.929771$

\begin{tabular}{|c|c|c|c|c|c|c|c|c|c|}
\hline \multirow{2}{*}{$i$} & \multicolumn{3}{|c|}{$K$} & \multicolumn{3}{|c|}{$E$} & \multicolumn{3}{|c|}{$I I$} \\
\hline & $\alpha_{i}$ & $\beta_{i}$ & $\eta_{i}$ & $\delta_{i}$ & $\epsilon_{i}$ & $\zeta_{i}$ & $\delta_{i}$ & $\epsilon_{i}$ & $\zeta_{i}$ \\
\hline $\begin{array}{l}0 \\
1 \\
2 \\
3 \\
4 \\
5 \\
\infty\end{array}$ & $\begin{array}{r}1.0000000 \\
.56932075 \\
.47083334 \\
.46562541 \\
.46561085 \\
.46551085\end{array}$ & $\begin{array}{r}0.13864149 \\
.37234593 \\
.46041748 \\
.46559628 \\
.46561085 \\
\end{array}$ & $\begin{array}{r}0.16350446 \\
.24446945 \\
.24998436 \\
.25000000 \\
\end{array}$ & $\begin{array}{l}7.2128480 \\
1.5290102 \\
1.0226226 \\
1.0000626 \\
1.0000000 \\
\end{array}$ & $\begin{array}{c}0.019221464 \\
.13864149 \\
.28532704 \\
.30450960 \\
.30461809 \\
\end{array}$ & $\begin{array}{l}1.0000000 \\
0.50961073 \\
.32412611 \\
.30472658 \\
.30461809 \\
.30461809\end{array}$ & $\begin{array}{l}0.11837970 \\
1.7275512 \\
1.0527845 \\
1.0005990 \\
1.0000001 \\
1.0000000\end{array}$ & $\begin{array}{c}60.929771 \\
7.3435239 \\
16.003806 \\
17.538501 \\
17.558747 \\
17.558750\end{array}$ & $\begin{array}{r}1.0000000 \\
30.964886 \\
19.154205 \\
17.579006 \\
17.558754 \\
17.558751 \\
17.558751\end{array}$ \\
\hline & \multicolumn{3}{|c|}{$\begin{aligned} K & =\frac{\pi}{2} \frac{1}{\alpha_{\infty}} \\
& =3.3736248\end{aligned}$} & \multicolumn{3}{|c|}{$\begin{aligned} E & =\frac{\pi}{2} \frac{\zeta \infty}{\alpha_{\infty}} \\
& =1.0276671\end{aligned}$} & \multicolumn{3}{|c|}{$\begin{aligned} I I & =\frac{\pi}{2} \frac{\zeta \infty}{\alpha_{\infty}} \\
& =59.236637\end{aligned}$} \\
\hline
\end{tabular}

TABLE 6. Computation of integrals $K, K-E$, and $I I-K$ for $x=x_{2}=8.09975 \mathrm{~cm}$

\begin{tabular}{|c|c|c|c|c|c|c|c|c|c|}
\hline \multirow{2}{*}{$i$} & \multicolumn{3}{|c|}{$K$} & \multicolumn{3}{|c|}{$K-E$} & \multicolumn{3}{|c|}{$I I-K$} \\
\hline & $\alpha_{i}$ & $\boldsymbol{\beta}_{i}$ & $\eta_{i}$ & $\delta_{i}$ & $\epsilon_{i}$ & $\zeta_{i}$ & $\delta_{i}$ & $\epsilon_{i}$ & $\zeta_{i}$ \\
\hline 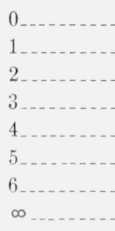 & $\begin{array}{c}1.0000000 \\
0.60621054 \\
.53355108 \\
.53106579 \\
.53106289 \\
\\
.53106289\end{array}$ & $\begin{array}{r}0.21242108 \\
.46089161 \\
.52858050 \\
.53105998 \\
.53106289 \\
\\
\end{array}$ & $\begin{array}{r}0.19007077 \\
.24767099 \\
.24999726 \\
.25000000 \\
\end{array}$ & $\begin{array}{l}4.7076307 \\
1.3152996 \\
1.0094036 \\
1.0000109 \\
10000000 \\
\end{array}$ & $\begin{array}{r}0.95487726 \\
.78757890 \\
.65362636 \\
.64311698 \\
.64309228 \\
\end{array}$ & $\begin{array}{r}\text { ค. } 0000000 \\
.47743863 \\
.63250877 \\
.64306757 \\
.64309228 \\
\\
.64309228\end{array}$ & $\begin{array}{l}0.077263226 \\
2.8548689 \\
1.289164^{`} \\
1.0162040 \\
1.0000646 \\
1.0000000 \\
1.0000000\end{array}$ & $\begin{array}{c}59.929771 \\
4.2982693 \\
10.956503 \\
13.654027 \\
13.847467 \\
13.848250 \\
13.848251 \\
\end{array}$ & $\begin{array}{l}0.0000000 \\
29.964886 \\
17.131578 \\
14.044041 \\
13.849034 \\
13.848251 \\
13.848251 \\
13.848251\end{array}$ \\
\hline & \multicolumn{3}{|c|}{$\begin{aligned} K & =\frac{\pi}{2} \frac{1}{\alpha_{\infty}} \\
& =2.9578348\end{aligned}$} & \multicolumn{3}{|c|}{$\begin{aligned} K-E & =\frac{\pi}{2} \frac{\zeta \infty}{\alpha_{\infty}} \\
& =1.9021608\end{aligned}$} & \multicolumn{3}{|c|}{$\begin{aligned} I I-K & =\frac{\pi}{2} \frac{\zeta \infty}{\alpha_{\infty}} \\
& =40.960838\end{aligned}$} \\
\hline
\end{tabular}


TABLe 7. Computation of integrals $K, K-E$, and $I I-K$ for $x=x_{3}=39.89876$

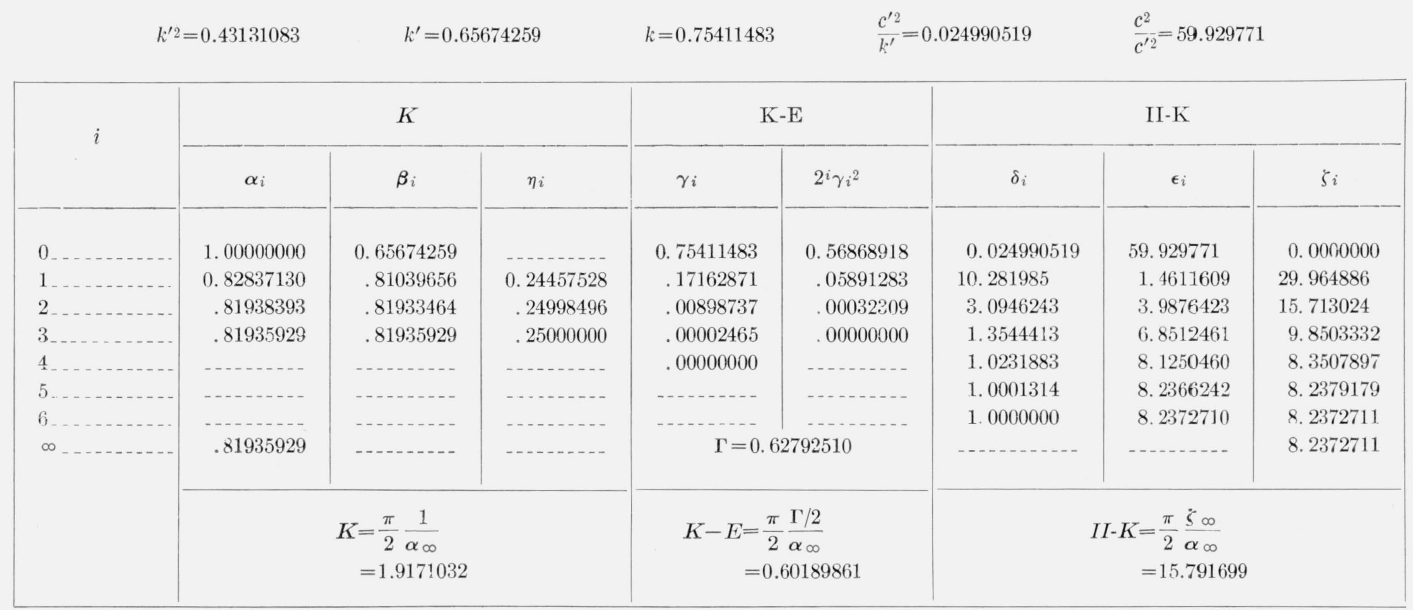

In the second example, illustrated in table 6, $\mathrm{K}, \mathrm{K}-\mathrm{E}$, and $\Pi-\mathrm{K}$ have been calculated for the case corresponding to $x=x_{2}$ in figure 17 . The constants have been chosen as indicated in table 4 .

In the final example, shown in table 7 , a combination of the King and Bartky methods has been used for computing the desired integrals. Here $\mathrm{K}$ has been calculated by use of the AGM scale, which is common to both methods; $\Pi-\mathrm{K}$ has been calculated by using Bartky's scales. To evaluate $\mathrm{K}-\mathrm{E}$, King's method is followed, introducing a $\gamma$ scale and a summation, $\Gamma$, defined as follows:

$$
\begin{gathered}
\gamma_{i+1}=\frac{1}{2}\left(\alpha_{i}-\beta_{i}\right) \\
\Gamma=\sum_{0}^{\infty} 2^{i} \gamma_{i}^{2},
\end{gathered}
$$

where the $\gamma$ scale starts with $\gamma_{0}=k$ and is identical with King's $c$ scale. From King's eq 27 it follows that

$$
\mathrm{K}-\mathrm{E}=\frac{\pi}{2 \alpha_{\infty}} \frac{\Gamma}{2}
$$

A review of the methods illustrated in tables 5, 6 , and 7 , indicates that there is no great difference in the amount of work required whichever method is used. The method shown in table 7 requires one less column of computation and is somewhat less subject to introduction of errors in calculation. Furthermore, in adapting the work to machine calculation it is possible to rearrange Jones' equation in a number of ways so that the end values of the various scales in table 7 are used directly. One such convenient arrangement of Jones' equation is

$$
M=\pi^{2} N_{x}\left\{\frac{r_{1}}{2} \frac{\Gamma}{\alpha_{\infty}}-\frac{(A-a)^{2}}{r_{1}} \frac{\zeta_{\infty}}{\alpha_{\infty}}\right\},
$$

where

$$
r_{1}^{2}=(A+a)^{2}+x^{2}
$$

and other quantities are as previously defined.

(c) Calculation of $M_{0}$

The mutual inductance, $M$, as given by eq 5 may now be evaluated, inasmuch as all the required elliptic integrals have been computed in tables 5, 6, and 7. The necessary data are assembled in table 8 . Using the values posted in the last column of this table, $M_{0}$ is calculated

\begin{tabular}{|c|c|c|c|c|c|}
\hline$x$ & $N_{\mathrm{x}}$ & $k$ & $\frac{K-E}{k^{2}}$ & $\frac{\mathrm{c}^{\prime 2}}{c^{2}}(I I-K)$ & M \\
\hline $\mathrm{cm}$ & & & & & \\
\hline 2. 49992 & 12.5 & 0.99034263 & 2. 3919342 & 0.9321413 & 5260.16 \\
\hline 8. 09975 & 40.5 & .97717812 & 1. 9920478 & .6834806 & 15074.30 \\
\hline 39.89876 & 199.5 & .75411483 & 1. 0583965 & .2635034 & 34809.80 \\
\hline
\end{tabular}
from eq 7 with the result:

$$
M_{0}=49991.32 \mathrm{cgsm} \text { units of inductance. }
$$

TABLE 8. Summary of computation 


\section{The Actual Inductor}

(a) General Procedure for Correcting $\mathrm{M}_{0}$

The effective value of mutual inductance for the actual inductor is given in terms of $M_{0}$, pertaining to the idealized inductor, by the expression

$$
M=\left[215 M_{0}\left(1+c_{s}\right)+\Delta M\right]\left(1+\Sigma c_{p}\right) .
$$

The quantities within the square bracket relate to the secondary, while the factor in round brackets takes account of departures of the primary winding from ideal conditions. The secondary winding consists of 218 turns, 215 of which are distributed in a regular manner, $8^{23 / 24}$ turns per layer in 24 layers. The distribution correction, $c_{s}$, arises from the fact that the average contribution of a turn in the 215 turns group differs somewhat from that of the hypothetical turn taken at the center of the channel for which $M_{0}$ was computed. The last three turns of the secondary are located in the twenty-fifth layer against one side of the channel and their contribution is evaluated separately and added as $\Delta M$. The primary corrections, $c_{p}$, involve five terms, each of which will be discussed in turn.

\section{(b) Corrections in Detail}

1. Correction for Secondary Distribution. The value of the secondary distribution correction, $c_{s}$, was determined by two methods. In both methods the calculations were made as if the secondary turns were circles instead of being turns on a multilayer helix. An estimate of the error so introduced gave a value of less than 1 part in $10^{8}$ of the total inductance, which is obviously negligible.

In the first method for calculating the value of the secondary distribution correction, $c_{s}$ was split into a component, $c_{s r}$, due to radial distribution and a component, $c_{s x}$, due to axial spread of the winding. In evaluating the component $c_{s r}$, all of the turns in each layer were considered to act as if concentrated as circles at the middle of each layer in the plane $x=0$. The mutual inductance between the primary and coaxial circles in the plane $x=0$ was calculated for 13 values of $A$ between the limits 24.8 and $27.7 \mathrm{~cm}$. The calculated values of $M$ were plotted against $A$ on a large sheet of coordinate paper, as illustrated on a small scale by figure 18. This figure shows that $\partial M / \partial A$ is zero

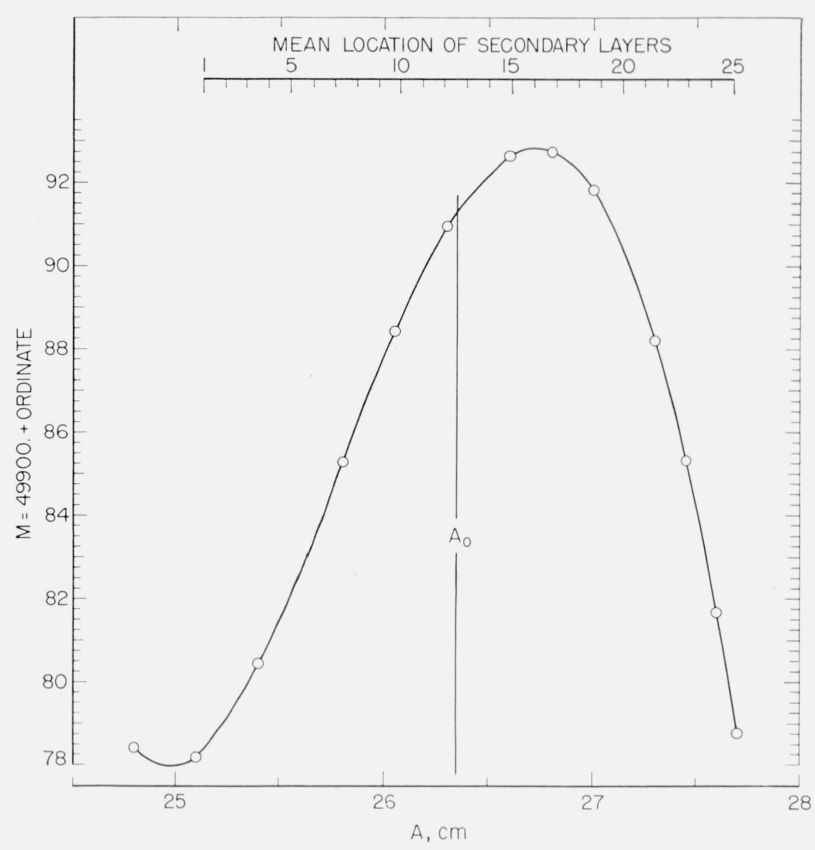

FiguRE 18. Mutual inductance between the whole primary and a single centrally located secondary circle of radius $A$.

$M$ in egsm units of inductance.

for two values of $A$, which is the feature sought by modification of the Campbell design. The approximate locations of the various secondary layers are indicated along the top edge of the figure. Inasmuch as $8^{23} / 24$ turns were considered to be concentrated at the center of each layer, the total inductance under this assumption is $8^{2} \frac{3}{24}$ times the sum of 24 values of $M$ taken from the curve at values of $A$ corresponding to the radii of each secondary layer from 1 to 24 , inclusive. The values of secondary radii are listed in table 3 . The total inductance summed in this manner is $10,747,241 \mathrm{cgsm}$ units of inductance. From the curve, the value of $M$ corresponding to one turn of radius $A_{0}$ is $49,991.31 ; 215$ turns concentrated at this radius would produce a total of $10,748,132$. From the relation $1+\mathrm{c}_{s r}=10,747,241 / 10,748,132$ the value of $c_{s \tau}$ was found to be $-83.0 \mathrm{ppm}$. This correction will later be applied to the basic value of $M_{0}$ given in eq 19 . It should perhaps be explained at this point that the curve of figure 18 was originally calculated in 1938 from dimensional data slightly different from those given in figure 17 . The curve has been brought up to date by means of calculated increments taking cognizance of dimensional changes; reliance is placed primarily upon the value of $M_{0}$ as currently calculated 
directly from 1948 dimensions rather than upon $M_{0}$ as taken from the curve, although the agreement between the two values is satisfactory.

The value of the correction $c_{s x}$ was determined from experimental data. To test the effect of the axial distribution of the turns, two flat "pancake" coils of 24 turns each were wound with inner and outer radii of 25.1 and $27.6 \mathrm{~cm}$, respectively. These two coils were mounted around the primary in place of the secondary, and the mutual inductance between the two flat coils, in series opposition, and the primary winding was determined. This was measured with the same circuit as for the absolute measurements, but instead of the 1-ohm standard resistor a low range adjustable resistor was used. This resistor was adjustable in steps of 0.01 microhm, and a series of balances was made with one pancake coil mounted $10 \mathrm{~mm}$ above the central plane of the primary as the second coil was raised from about $6 \mathrm{~mm}$ below the central plane. This passed through the region normally occupied by the secondary and gave a measure of the change in mutual inductance as the 24 turns were moved in either direction from the central plane. The change in resistance required to balance the circuit plotted against the axial position of the movable coil as measured from the fixed is shown in figure 19. The lowest part of the curve corre-

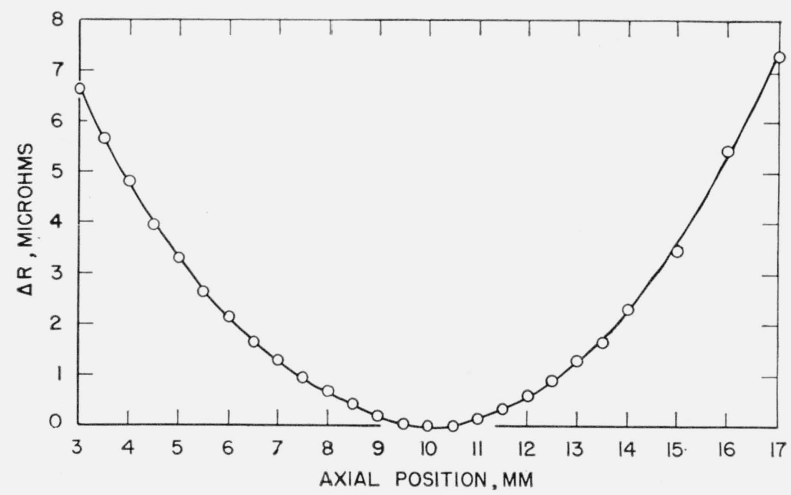

Figure 19. Experimental data for determination of correction for axial distribution of secondary.

sponds to the position of the central plane of the secondary. Changes in ordinate when multiplied by 1,000 and divided by $4 n$ (see eq 3 ) give the change in inductance for one circle in each of the 24 layers as the group moves in either direction from the central plane.

The effect of the axial distribution of the secondary on the mutual inductance was calculated as follows. The secondary was considered to consist of 24 circles in the central plane, 24 circles 0.9 $\mathrm{mm}$ on each side of the central plane, and also 24 circles at 1.8, 2.7, and $3.6 \mathrm{~mm}$ each side of this plane. From the curve, figure 19, were determined increases in $R$ at $0.9,1.8 \mathrm{~mm}$, etc., each side of the minimum. These changes in $R$ when multiplied by $1000 / 4 n$ gave the inductance increments for the several groups at their respective distances from the central plane. The sum of these inductance increments was $77 \mathrm{cgsm}$ units of inductance. When expressed in proportional parts of $215 M_{0}$ this gave the result $c_{\mathrm{sx}}=+7.2 \mathrm{ppm}$. Combining the radial and axial components of the secondary distribution correction, $c_{\mathrm{s}}=-83.0+7.2=-75.8 \mathrm{ppm}$.

In calculating the secondary distribution correction by the second method, use was made of a formula derived by Chester Snow of this Bureau. In terms of his formula the distribution correction can be calculated from eq 21. This is not a general expression but an approximation which applies only to the particular inductor, as certain terms have been neglected because of the flux distribution around the secondary, and others have been neglected because they involve the sixth or higher power of the small quantities $b / A$ or $c / A$. In eq $21, M$ is a function of $x$ and $A$, and is the mutual inductance between the primary and a circle located within the bounds of the secondary channel. All other quantities are as previously defined. The chief labor involved in calculating $c_{s}$ by this formula arises in connection with the evaluation of the various partial derivatives involved; these are to be determined at the point $x=0, A=A_{0}$. These derivatives may be evaluated by means of eq 22 to 28 , inclusive. In eq $22, p$ is the nominal pitch of the primary helix and $Y_{2}(k)$ is obtained from Nagaoka and Sakurai's [11] tables, where it is tabulated against $k^{2}$ under the heading $(\sqrt{\mathrm{A} a} / z) F$. The nature of the function $F(x)$ appearing in eq 23 need not be entered into here; its value is to be computed from eq 24 . The function $Y_{1}(k)$ is obtained from Nagaoka's tables, where it is tabulated against $k^{2}$ under the heading $M / \sqrt{A a}$. The nature of the function $G(x)$ appearing in eq 25 need not be discussed; it is evaluated by means of eq 26, wherein $Y_{2}^{\prime}(k)$ and $Y_{2}^{\prime \prime}{ }^{\prime}(k)$ are to be evaluated by use of eq 27 and 28 . In table 9 the data required to calculate $c_{s}$ have been compiled. 


$$
\begin{aligned}
& c_{s}=\frac{1}{M_{0}}\left\{-\frac{1}{6}\left[c^{2}-b^{2}-\frac{c^{4}}{10 A_{0}{ }^{2}}\right] \frac{\partial^{2} M}{\partial x^{2}}+\left[\frac{b^{4}}{120}\right] \frac{\partial^{4} M}{\partial x^{4}}-\left[\frac{c^{2}\left(3 c^{2}-10 b^{2}\right)}{360}\right] \frac{\partial^{4} M}{\partial A^{2} \partial x^{2}}\right\} \\
& \frac{\partial^{2} M}{\partial x^{2}}=\frac{2}{p \sqrt{A_{0} a_{0}}}\left\{-x_{1} Y_{2}\left(k_{1}\right)+x_{2} Y_{2}\left(k_{2}\right)-x_{3} Y_{2}\left(k_{3}\right)\right\} \\
& \frac{\partial^{4} M}{\partial x^{4}}=2\left\{F\left(x_{1}\right)-F\left(x_{2}\right)+F\left(x_{3}\right)\right\} \\
& F(x)=\frac{x k^{3}}{4 p k^{\prime^{2}}\left(A_{0} a_{0}\right)^{3 / 2}}\left\{\frac{3}{4}\left[k\left(1+2 x-\frac{3 x^{2} k^{2}}{4 A_{0} a_{0}}\right)-\frac{x^{2} k^{3}\left(5-k^{2}\right)}{4 A_{0} a_{0} k^{\prime 2}}\right] Y_{1}(k)+\left[2\left(\frac{2}{k}-k\right)\left(1+2 x-\frac{3 x^{2} k^{2}}{4 A_{0} a_{0}}\right)-\right.\right. \\
& \left.\left.\frac{x^{2} k^{3}\left(\frac{15}{k^{2}}-9+2 k^{2}\right)}{4 A_{0} a_{0} k^{\prime 2}}\right] Y_{2}(k)\right\} \\
& \frac{\partial^{4} M}{\partial A^{2} \partial x^{2}}=2\left\{G\left(x_{1}\right)-G\left(x_{2}\right)+G\left(x_{3}\right)\right\} \\
& G(x)=\frac{x}{4 p A^{2}{ }_{0} \sqrt{A_{0} a_{0}}}\left\{-3 Y_{2}(k)+\left[\frac{A_{0} k^{3}}{a_{0}}+\left(1-\frac{\left[A_{0}+a_{0}\right] k^{3}}{2 a_{0}}\right)\left(4+\frac{3\left[A_{0}+a_{0}\right] k^{2}}{2 a_{0}}\right)\right] Y_{2}^{\prime}(k)-\right. \\
& \left.\left[1-\frac{\left(A_{0}+a_{0}\right) k^{3}}{2 a_{0}}\right]^{2} Y_{2}^{\prime \prime}(k)\right\} \\
& Y_{2}^{\prime}(k)=\frac{\frac{3}{4} k Y_{1}(k)+2\left[\frac{2}{k}-k\right] Y_{2}(k)}{k^{\prime^{2}}} \\
& Y_{2}^{\prime \prime}(k)=\frac{1}{k^{\prime 4}}\left[\frac{3}{4}\left(5-k^{2}\right) Y_{1}(k)+\left(\frac{15}{k^{2}}-9+2 k^{2}\right) Y_{2}(k)\right]
\end{aligned}
$$

TABLE 9. Summary of data required for calculating $c_{s}$ by Snow's method

\begin{tabular}{|c|l|l|l|}
\hline & & & \\
$A_{0}=26.348 \mathrm{~cm}$ & $x_{1}=2.500 \mathrm{~cm}$ & $x_{2}=8.100 \mathrm{~cm}$ & $x_{3}=39.90 \mathrm{~cm}$ \\
$a_{0}=20.364 \mathrm{~cm}$ & $k_{1}=0.9903$ & $k_{2}=0.9772$ & $l_{3}=0.7541 \mathrm{~cm}$ \\
$p=0.2 \mathrm{~cm}$ & $k_{1}^{2}=.9808$ & $k_{2}^{2}=.9549$ & $l_{3}^{2}=.5687 \mathrm{~cm}$ \\
$b=.41 \mathrm{~cm}$ & $Y_{2}=148.5$ & $Y_{2}=56.90$ & $Y_{2}=1.256 \mathrm{~cm}$ \\
$c=1.273 \mathrm{~cm}$ & $Y_{1}=17.55$ & $Y_{1}=12.60$ & $Y_{1}=1.892 \mathrm{~cm}$ \\
\hline
\end{tabular}

By carrying out the necessary calculations it was found that

$\frac{\partial^{2} M}{\partial x^{2}}=+17.02, \quad \frac{\partial^{4} M}{\partial x^{4}}=-16.97, \quad \frac{\partial^{4} M}{\partial x^{2} \partial A^{2}}=-15.69$.

When these values were employed in eq 21 the result was

$c_{s}=\frac{1}{M_{0}}\{-4.120-0.0040+0.2246\}=\frac{1}{M_{0}}\{-3.899\}$.

Using the value of $\mathrm{M}_{0}$ given in eq $19, c_{s}=-78.0$ ppm. This value differs by only $2.2 \mathrm{ppm}$ from the value obtained by the first method. It is, however, considered to be somewhat less reliable than the figure obtained by the first method because the turns were here assumed to be uniformly distributed over the secondary channel, whereas in the first method, the measured diameter of each layer was used in the calculations. The value that will be used for $c_{s}$ is therefore that obtained by the first method.

2. Increment in $M$ Due to Three Off-Center Turns. Equation 5, which is used in calculating the mutual inductance, gives the mutual inductance between a helix and a coaxial circle located in the plane of one end of the helix. For secondary turns on the plane $x=0$ the inductance is calculated for the part of the primary lying on one side of the plane and the result is doubled to take into account the other half. For secondary turns not on the plane $x=0$ the two ends of the primary are not equal and their contributions must be calculated separately and added. This calculation has been made for the three off-center turns in the 
twenty-fifth layer, and it was found that their inductance totaled $4.74 \mathrm{cgsm}$ units of inductance more than would have been obtained with three turns on the central plane.

It is assumed that the difference between the inductance of the off-center turns and three turns on the central plane will remain constant with time, although the inductance of turns on the central plane may change slowly with changes in primary dimensions. From the large scale plot of figure 18 the value of 49979.75 is found for $M_{25}$. The total contribution of the three extra turns is therefore $\Delta M=3(49979.75)+4.74=$ 149,944 cgsm units of inductance.

3. Correction for Primary-Current Distribution. In the sections to follow, dealing with corrections arising out of imperfections in the primary winding, it will be considered that all 218 secondary turns act as if concentrated on the circle $A=A_{0}$ at $x=0$, i. e., $M=218 M_{0}$. The error in $M$ due to this assumption is entirely negligible in its effect upon the magnitude of small primary corrections computed on this basis.

In the resistance-measurement method described is this paper the effective value of the mutual inductance needs to be known at that moment in each cycle when the secondary connections are reversed. During this portion of the cycle the current in the primary of the inductor is extremely constant; for the present, only the steady-state current distribution over the cross section of the primary wire is of concern. This current distribution may be considered under three headings: (a) uniform distribution, (b) the so-called "natural" distribution, in which current distribution is determined solely by the variation in length of the various filaments in the conductor, (c) a closer approximation to the actual current distribution in which the variation in resistivity over the cross section of the wire, as well as variations in path length, is taken into account. Stresses introduced in the wire during winding change the resistivity of the conductor by almost 1 percent over its cross section, and as a consequence the current distribution is influenced appreciably. It will first be shown how the magnitude of the current distribution correction is determined for case (c), after which the results for cases (a) and (b) may readily be determined as special cases for comparative purposes.
The problem is approached by dividing the cross section of the primary wire into an odd number of elements of equal area $q_{0}, q_{1}, q_{2} \ldots q_{n}$ constructed by drawing chords parallel to the axis of the winding as indicated in figure 20. Nine

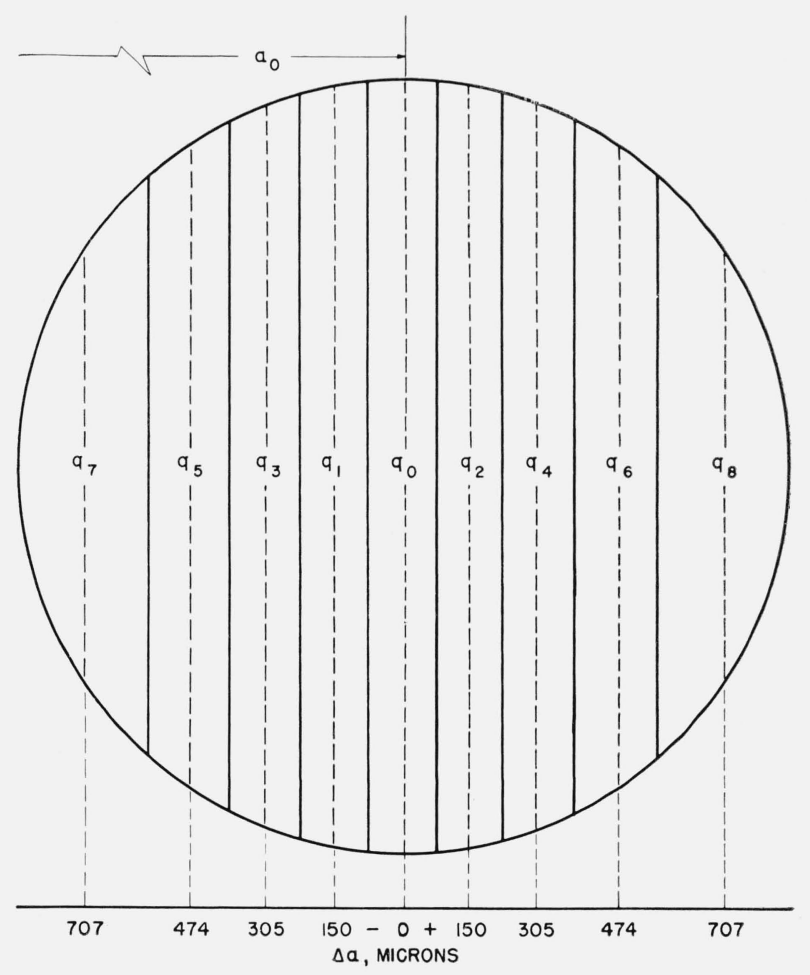

FIGURE 20. Cross section of primary wire divided into nine elements of equal area, for use in calculation of primary current-distribution correction.

elements are sufficient for the present purpose. The distance in microns from the center of the wire to the center of each element of area is shown on the figure. The next step is to determine the contribution to the total flux linkages made by the current $i_{j}$ flowing in the elemental area $q_{i}$, the center of which has a mutual inductance $M_{\text {, with }}$ respect to a single-turn secondary located at the center of the secondary channel. This contribution is $M_{j} i_{j}$ and the total flux linkages are found by adding the contributions produced by each of the nine sections. The correction, $c$, for current distribution is determined from the defining equation

$$
M_{0}(1+c) I=\sum_{0}^{n} M_{j} i_{j}
$$

Here $M_{0}$ is the basic value of mutual inductance as given in eq $19 ; I$ is the total primary current 
and is the sum of the unequal current elements $i_{0} \ldots i_{n}$. The factor $(1+c)$ is that by which $M_{0}$ must be modified in order that the correct total effective flux linkages be arrived at when $I$ is considered to be concentrated on a helical filament of radius $a_{0}$.

In order to proceed with the solution for $c$ from eq 29 , it is necessary to determine how $M$ and $i$ vary over the cross section of the wire. The manner in which $M$ varies is given by a Taylor's series expansion of $M$ as a function of $\Delta a$ in the vicinity of $a_{0}$. Inasmuch as the higher order derivatives of $M$ with respect to $a$ become cumbersome, it is more convenient to evaluate the desired coefficients in the following manner. $M$ and $\partial M / \partial a$ are calculated for three values of $a$ using the following equations:

$$
\begin{aligned}
& \frac{\partial M}{\partial a}=2\left\{\left(\frac{\partial M}{\partial a}\right)_{x_{1}}-\left(\frac{\partial M}{\partial a}\right)_{x_{2}}+\left(\frac{\partial M}{\partial a}\right)_{x_{3}}\right\} \\
& \frac{\partial}{\partial a} M=\frac{\pi^{2}}{\alpha_{\infty}} \frac{N_{x}}{r_{1}} \frac{4 A a}{(A+a)}\left\{1+\frac{A-a}{2 A} \zeta_{\infty}\right\}
\end{aligned}
$$

In the last equation $\alpha_{\infty}$ and $\zeta_{\infty}$ are the end terms in the scales used for computing $K$ and $\Pi-K$, as illustrated in table 7. From the results of calculations made by taking $a$ equal to $a_{0}+0.05 \mathrm{~cm}$, $a_{0}$, and $a_{0}-0.05 \mathrm{~cm}$, it is possible to evaluate the coefficients of the Taylor's series up to and including the third-order term, as follows:

$$
\begin{aligned}
M= & M_{0}+4703.07(\Delta a)+107.0(\Delta a)^{2}+ \\
& 3.5(\Delta a)^{3}+\ldots
\end{aligned}
$$

This equation will be applied to the present problem in the more convenient form:

$$
\begin{aligned}
M_{j} & =M_{0}\left\{1+\left[94078\left(\Delta a_{j}\right)+2140\left(\Delta a_{j}\right)^{2}+70\left(\Delta a_{j}\right)^{3}\right]\right. \\
& \equiv M_{0}\left\{1+\lambda_{j}\right\}
\end{aligned}
$$

where, in both equations, $\Delta a$ is in centimeters, and $\lambda$ is in parts per million.

The manner in which $i$ varies over the cross section of the wire may be arrived at by writing

$$
i_{j}=\frac{e q_{j}}{\rho_{j} l_{j}}
$$

Here $e$ is considered to be the voltage drop across one turn of the helix, $\rho_{j}$ the resistivity of the conductor at area element $q_{j}$, and $l_{j}$ the length of a one-turn filament of the conductor at a distance of
$a_{0}+\Delta a_{j}$ from the axis of the winding. The current $i_{j}$ is desired in terms of that in the central element of area $q_{0}$, and inasmuch as all of the area elements are equal, eq 34 may be expressed in the form

$$
\begin{aligned}
i_{j} & =\frac{e q_{0}}{\rho_{0}\left(\frac{\rho_{j}}{\rho_{0}}\right) l_{0}\left(1+\frac{\Delta a_{j}}{a_{0}}\right)} \\
& \equiv \frac{i_{0}}{\left(1+\mu_{j}\right)\left(1+\nu_{j}\right)}
\end{aligned}
$$

From this last equation the total current, I, may be expressed as follows:

$$
I=i_{0} \sum_{0}^{n} \frac{1}{\left(1+\mu_{j}\right)\left(1+\nu_{j}\right)}
$$

Combining eq 33, 35, and 36, eq 29 may now be written in the form

$$
M_{0}(1+c) i_{0} \sum_{0}^{n} \frac{1}{\left(1+\mu_{j}\right)\left(1+\nu_{j}\right)}=\sum_{0}^{n} \frac{M_{0}\left(1+\lambda_{j}\right) i_{0}}{\left(1+\mu_{j}\right)\left(1+\nu_{j}\right)}
$$

or

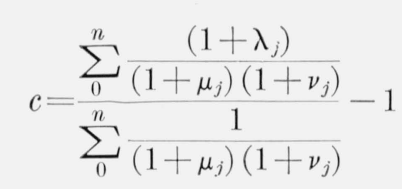

In order to evaluate $c$, the problem that remains is to determine how the resistivity varies over the cross section of the wire, in order that the coefficients $\mu_{j}$ may be found. Bridgeman [12] has shown that in addition to any changes in dimensions, a copper wire will change in resistivity when subjected to stress. He found that a pressure of $1 \mathrm{~kg} / \mathrm{mm}^{2}$ would decrease the resistivity by 170 ppm. In addition, it has also been shown experimentally [13] that the effects of tension and compression are the same numerically, but opposite in sign. At this point it is appropriate to mention that after the primary wire had been wound onto the porcelain form in one continuous helix, and secured at the desired locations, two lengths of wire were removed from the cylinder to provide the blank regions on each side of the central section. This wire, when relieved of tension, retained a radius of curvature of about $28 \mathrm{~cm}$, indicating that in the process of winding portions of it had been stressed beyond the elastic limit, i. e., that maximum stresses in the neighborhood of $20 \mathrm{~kg} / \mathrm{mm}^{2}$ were involved.

Figure 21 presents stress-strain data taken by the Engineering Mechanics Section of this Bureau 


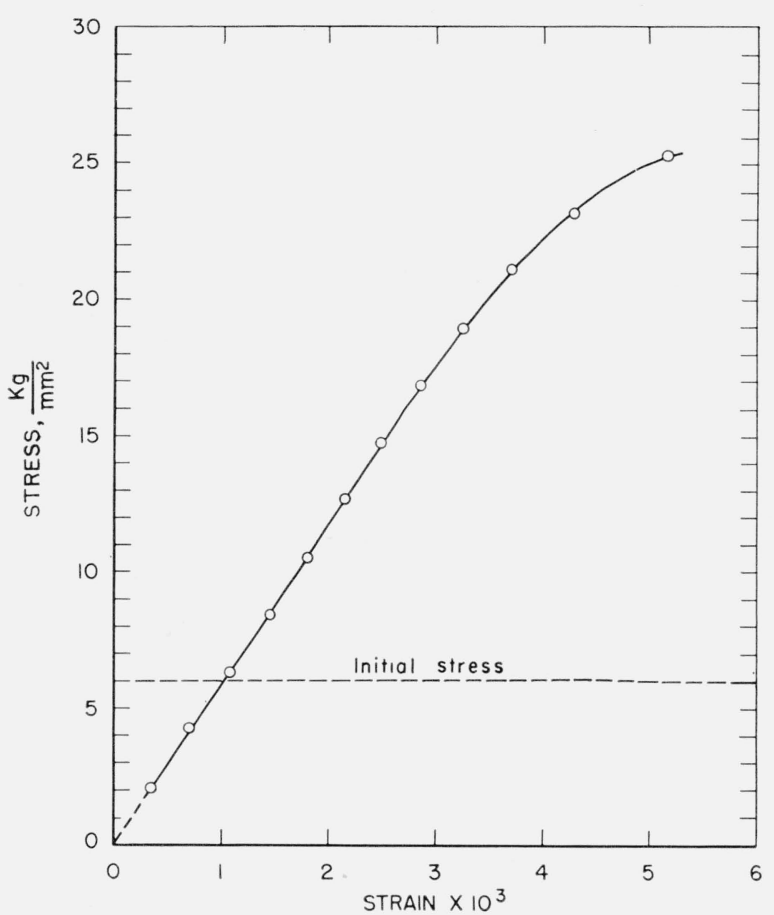

Figure 21. Stress-strain diagram for copper wire used in winding primary of mutual inductor.

on samples of the copper wire used for winding the primary. The ordinate $6 \mathrm{~kg} / \mathrm{mm}^{2}$ represents the stress applied to the wire used in winding the cylinder when the wire was pulled through the final diameter-reducing die and onto the porcelain form. The curve begins to depart from a straight line at about $18 \mathrm{~kg} / \mathrm{mm}^{2}$ and in what follows this stress will be taken as the proportional limit of the wire. As the wire was curved around the cylinder in the winding process the initial stress was modified by the added tension or compression in the fibers either side of the neutral plane. The location of the neutral plane is somewhat uncertain, but in view of the manner in which the wire is supported in the porcelain groove it is believed that it lies close to the center of the wire, perhaps slightly in the direction toward the porcelain. For the present example the neutral plane will be considered to pass through the center of the wire. In this case the strains at various distances from the center are $\Delta a / a_{0}$, and the dotted curve of figure 22 can be obtained from figure 21 after calculating strain in terms of $\Delta a$. This dotted curve represents the stress distribution for the case of simple bending. The effect of the drawing tension is to displace the curve to the position indicated by the solid curve. Curve $A$ of figure 23 is obtained from the solid curve of figure 22 by use of the following expression involving Bridgeman's resistivity-stress coefficient:

$$
\frac{\Delta \rho}{\rho}=170 \sigma
$$

This equation gives the change in resistivity in proportional parts of $\rho$, where $\rho$ is considered to be the resistivity of the wire at that point in its cross section where the stress, $\sigma$, is taken as zero, i. e., at the point where the solid curve of figure 22 crosses the horizontal axis. In figure 23 the horizontal branches $B$ of the curve are drawn on the assumption that yielding started suddenly when the fiber stress reached $18 \mathrm{~kg} / \mathrm{mm}^{2}$ and plastic flow prevented the stress from rising above this figure. The line $C$ is obtained by extending the straight portion of curve $A$. By using the information contained in figure 23 , it is now possible to calculate the ratio of the resistivity at the center of area element $q_{j}$ to that at the center of area element $q_{0}$, using the relation

$$
\frac{\rho_{j}}{\rho_{0}}=\frac{1+y_{j}}{1+y_{0}} \equiv 1+\mu_{j},
$$

where $y$ is an ordinate of the curve of figure 23 .

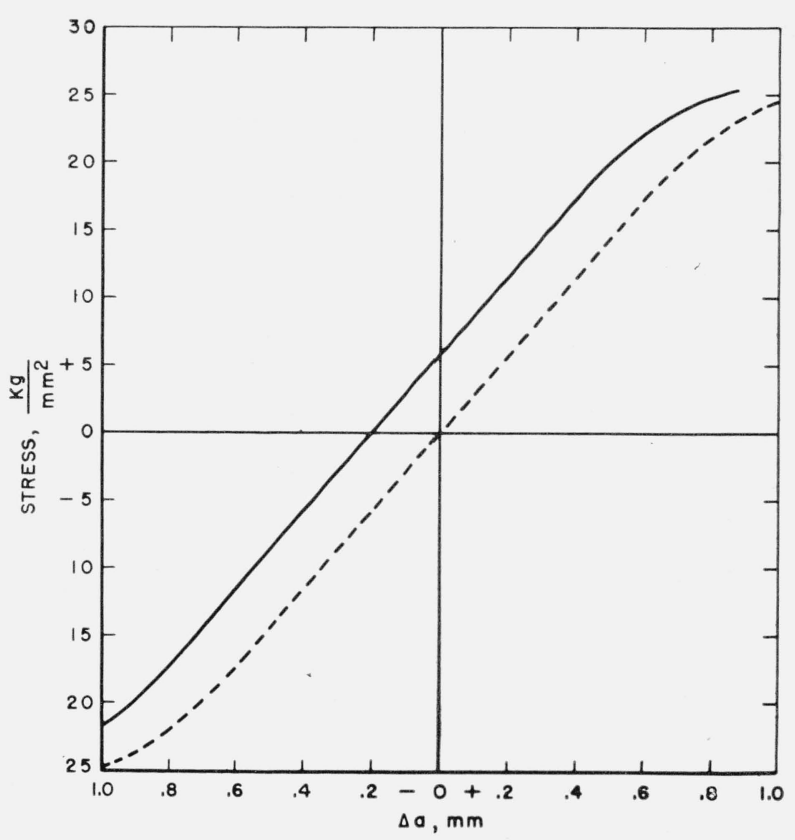

FIGURE 22. Stress in primary wire as a function of distance from center of wire. 


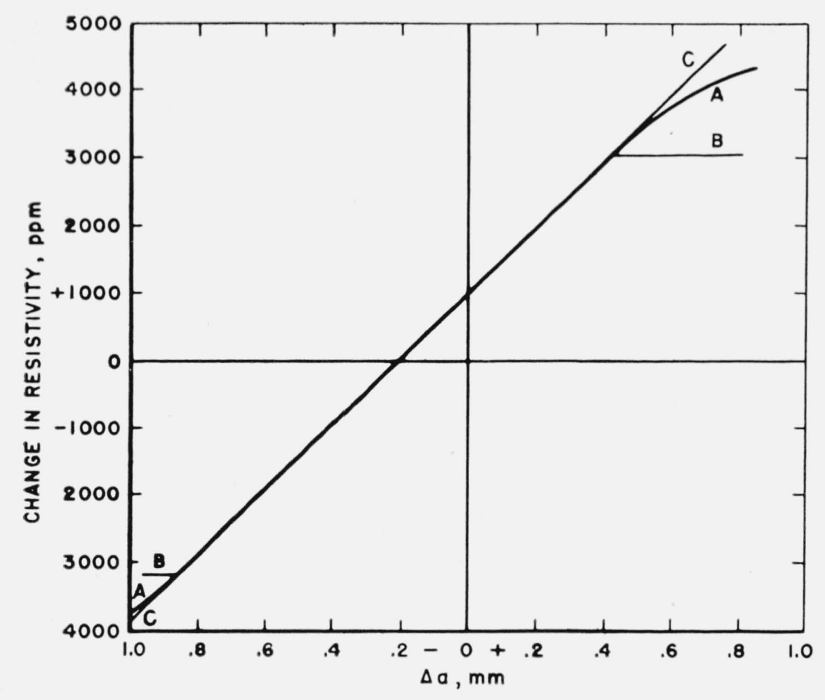

FIGURE 23. Variation in resistivity over the cross section of the primary wire.

All of the information required for calculating the value of the current-distribution correction $c$ is now available. This information is assembled in table 10 and pertains to the situation where the resistivity variation over the cross section of the wire is as indicated by curve $A$ of figure 23 . Applying eq 37 , the result $c=(9.0005737 / 9.0006901)-$ $1=-12.9 \mathrm{ppm}$ is obtained. The value of the correction for the natural current distribution, case (b), is obtained by setting $\mu=0$, in which instance the factors and summations are as given in columns 8 and 9 of the table. The value of the correction is $c=-4.6 \mathrm{ppm}$. Lastly, for uniform current distribution, case (a), both $\mu$ and $\nu$ should be set equal to zero, whence $c=+4 \mathrm{ppm}$. It is thus evident that the value of the current-distribution correction depends decidedly upon the initial assumptions. In particular, if the current distribution were assumed to be uniform the effective value of $M$ would be in error by some 17 parts per million. It may be argued that the current distribution is uncertain because of the effect of terminal connections. Experience with precise resistance measurements has indicated, however, that in long conductors the resistance between potential points located two or three wire diameters away from the current connections is independent of the particular manner in which the current is introduced, i. e., a short distance away from the current connections the current distribution is uninfluenced by the disposition of the current connections. It is realized that the large range in the values for the correction here reported is a consequence of the large wire used in this work. However, the method of analysis which has been described is applicable to wire of any size. 'To the best of the authors' knowledge the effect of variation of resistivity upon current distribution, and consequently upon the effective value of $M$, has not previously been investigated.

TABLE 10. Data for calculating current-distribution corrections

\begin{tabular}{|c|c|c|c|c|c|c|c|c|}
\hline 1 & 2 & 3 & 4 & 5 & 6 & 7 & 8 & 9 \\
\hline$j$ & $\Delta a, \mathrm{~cm}$ & $1+\lambda_{j}$ & $1+\mu_{j}$ & $1+\nu_{j}$ & $\frac{1+\lambda_{j}}{\left(1+\mu_{j}\right)\left(1+\nu_{j}\right)}$ & $\frac{1}{\left(1+\mu_{j}\right)\left(1+\nu_{j}\right)}$ & $\frac{1+\lambda_{j}}{1+\nu_{j}}$ & $\frac{1}{1+\nu_{j}}$ \\
\hline $0 \ldots$ & 0 & 1. 0000000 & 1. 0000000 & 1. 0000000 & 1. 0000000 & 1. 0000000 & 1. 0000000 & 1. 0000000 \\
\hline $1 \ldots$ & -0.0150 & 0.9985893 & 0.9992438 & 0. 9992634 & 1. 0000816 & 1. 0014944 & 0. 9993254 & 1. 0007371 \\
\hline $2 \ldots$ & +.0150 & 1. 0014117 & 1. 0007323 & 1. 0007366 & 0. 9999424 & 0.9985328 & 1. 0006746 & 0. 9992639 \\
\hline $3 \ldots$ & -.0305 & 0. 9971326 & 0.9984646 & 0. 9985022 & 1. 0001015 & 1. 0029774 & 0.9986283 & 1. 0015000 \\
\hline $4 \ldots$ & +.0305 & 1. 0028714 & 1. 0015015 & 1. 0014978 & 0. 9998703 & 0. 9970075 & 1. 0013715 & 0. 9985044 \\
\hline $5 \ldots \ldots$ & -.0475 & 0.9955455 & 0. 9976355 & 0.9976723 & 1. 0002333 & 1. 0047088 & 0.9978682 & 1. 0023331 \\
\hline $6 \ldots$ & +.0475 & 1. 0044641 & 1. 0022807 & 1. 0023277 & 0. 9998511 & 0. 9954075 & 1. 0021314 & 0.9976777 \\
\hline $7 \ldots$ & -.0707 & 0. 9933594 & 0.9964867 & 0. 9965281 & 1. 0003348 & 1. 0070220 & 0. 9968203 & 1. 0034840 \\
\hline $8 \ldots$ & +.0707 & 1. 0066620 & 1. 0030199 & 1. 0034719 & 1. 0001587 & 0. 9935397 & 1. 0031791 & 0. 9965401 \\
\hline$\Sigma$ & & 9. 0000360 & & & 9. 0005737 & 9. 0006901 & 8. 9999988 & 9. 0000403 \\
\hline
\end{tabular}


The correction has also been calculated for the cases pertaining to curves $B$ and $C$ of figure 23, with the results $c=-12.0 \mathrm{ppm}$ and $-13.3 \mathrm{ppm}$, respectively. In addition, calculations have been made with the cross section of the wire divided into 19 elements of area instead of 9 as used in this discussion; the results did not differ appreciably from those given above. When the primary was wound in 1938 it was realized that the stress in the outer fibers of the conductor exceeded the elastic limit, and that therefore the exact shape of curve $A$, figure 23, was uncertain because of the possibility of cold creep of the metal accompanied by some stress relief. It was believed that the results using curves $B$ and $C$ represented limiting cases and that as time went on the correction pertaining to curve $B$ would be most representative of actual conditions. If any appreciable stress relief occurred, an increase in diameter and a decrease in pitch would be expected. During the decade 1938 to 1948 the mean diameter and pitch both increased by approximately the same proportional amount, and there has been no definite evidence indicating that there has been any appreciable change in the wire stresses subsequent to the 1938 dimensional measurements. The intermediate value obtained by use of curve $\mathrm{A}$ is considered to be the most probable value of $c$; the current-distribution correction is therefore taken as $-12.9 \mathrm{ppm}$.

4. Correction for Distortion of Primary Wire. The phenomena associated with the drawing and bending of the primary wire as it is wound onto the porcelain cylinder are very complicated. An exact solution for the distortion of the wire cannot be given, but the distortion is believed to be small and consequently approximate methods are considered to be adequate.

A tension of $6 \mathrm{~kg} / \mathrm{mm}^{2}$ was employed when the wire was drawn onto the cylinder. This stress produced a slight but uniform elastic reduction in wire diameter and also modified the density. These effects were taken into account when the mean wire diameter was determined from length, mass, and density data. Stresses produced by bending do not significantly alter the mean density of the wire, but they change the resistivity of the wire and possibly distort its cross-sectional shape. The consequences of resistivity changes have been analyzed in connection with the currentdistribution correction; the problem of distortion will now be considered.
The theory of a bar bent by couples has been given by Saint-Venant and is dealt with by Love [14]. As a solution of the pertinent differential equations, the latter gives displacement equations equivalent to the following, wherein only obvious changes in notation have been made:

$u=\frac{1}{2 a}\left(z^{2}+\mu x^{2}-\mu y^{2}\right), \quad v=\mu \frac{x y}{a}, \quad w=-\frac{x y}{a}$.

Here $a$ is the radius of curvature, $\mu$ is Poisson's ratio, and $u, v$, and $v$ are displacements parallel to the $x, y$, and $z$ axes, respectively. These equations are general, and apply to a bar of any cross-sectional shape, provided that stresses exist in the axial or $z$ direction only, and that they vary linearly with $x$. When these equations are applied to a wire that is initially straight, and circular in cross section, one is led to the rather surprising conclusion that the section remains circular after the wire is bent. However, the present problem is complicated by the fact that the conditions stipulated in connection with eq 40 are not entirely fulfilled. The condition that stresses exist in the $z$ direction only is fulfilled to good approximation but because the extreme fiber bending-stress exceeds the elastic limit, the axial stress is not a linear function of $x$ over the whole cross section of the wire. Some distortion therefore occurs, and an attempt to estimate the upper limit of its magnitude and resultant effect on the value of $M$ is made by the following approximate method.

Reference is made to figure 24 where a righthand system of coordinates corresponding with Love's figure 10 is used. Coordinates are measured from the center of the undistorted wire. If the elastic limit of the wire is taken as 18 $\mathrm{kg} / \mathrm{mm}^{2}$, this stress will be reached in tension at the plane $x=-400$ microns, where, as before, the neutral plane is considered to pass through the center of the undistorted wire. Material located between $x=-400$ and $x=+850$ microns is not stressed beyond the elastic limit and is considered to be perfectly elastic with Poisson's ratio equal to 0.3 , while the segment of wire between $x=-400$ and $x=-850$ is considered to act as a perfectly plastic (incompressible) material with Poisson's ratio equal to 0.5 . The segment was divided into nine smaller segments by means of equally spaced chords parallel to $0-Y$. From the diminution in dimensions of each small segment the displacement $f-f^{\prime}$ has been calculated and found 


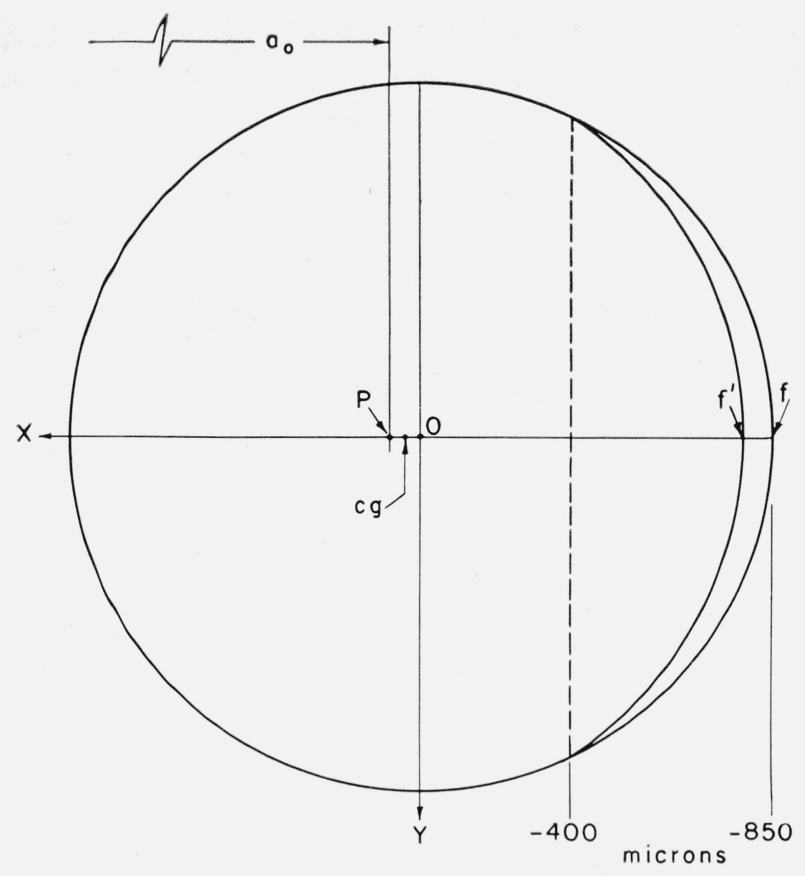

FIgURE 24. Sketch illustrating in exaggerated form the distortion of the primary wire.

to be 0.2 micron. Inasmuch as each small segment suffers a slight reduction in area the center of gravity of the whole cross-sectional area is shifted, in the direction shown, by a small amount 0 to $\mathrm{eg}$, which has been calculated and found to be 0.1 micron. The basic value of mutual inductance, $M_{0}$, was calculated to the point, $P$, which was assumed to be the center of the wire, the distance $P-f^{\prime}$ having been taken as one-half the mean undistorted wire diameter. With respect to the point $P$ the center of gravity is 0.1 micron further removed from the axis of the winding. The mutual inductance must therefore be increased by an amount corresponding to an increase in $a_{0}$ of 0.1 micron. Using eq 33 the value of this correction is found to be $+0.9 \mathrm{ppm}$. This correction has been calculated for several assumed values of elastic limit with results not different from the above by more than a few parts in $10^{7}$. If no portion of the wire was subjected to stress beyond the elastic limit, the value of the correction for distortion would be zero. The conditions assumed in the approximate calculation were limiting conditions; the actual conditions are probably intermediate. The value of the distortion correction is therefore taken as $+0.5 \mathrm{ppm}$.

5. Corrections for Irregularities in Primary Diameter and Pitch. In order to correct for irregularities in diameter and pitch of the primary winding the partial derivatives of $M$ with respect to $a$ and $x$ are required for coaxial circles. One circle of radius $A$ is fixed in the plane $x=0$ and represents the secondary, while the second circle of radius $a$, located at various axial positions, represents a turn, or group of turns, in the primary helix. When Maxwell's equation for the mutual inductance between two coaxial circles [15] is differentiated with respect to $a$ and $x$, the following expressions are obtained:

$$
\begin{gathered}
\frac{\partial M}{\partial a}=\frac{4 \pi a}{r_{1}}\left\{(\mathrm{~K}-\mathrm{E})+\frac{2 A(A-a)}{r_{2}{ }^{2}} \mathrm{E}\right\} \\
\frac{\partial M}{\partial x}=\frac{4 \pi x}{r_{1}}\left\{(\mathrm{~K}-\mathrm{E})-\frac{2 A a}{r_{2}{ }^{2}} \mathrm{E}\right\}
\end{gathered}
$$

where $r_{1}{ }^{2}=(A+a)^{2}+x^{2}$ and $r_{2}^{2}=(A-a)^{2}+x^{2}$.

These equations were evaluated as a function of $x$ for $A=A_{0}=26.348 \mathrm{~cm}, a=a_{0}=20.364 \mathrm{~cm}$, and plotted in figure 25 . For values of $x$ between 0 and $25 \mathrm{~mm}$ the values of $\mathrm{K}$ and $\mathrm{E}$ were calculated

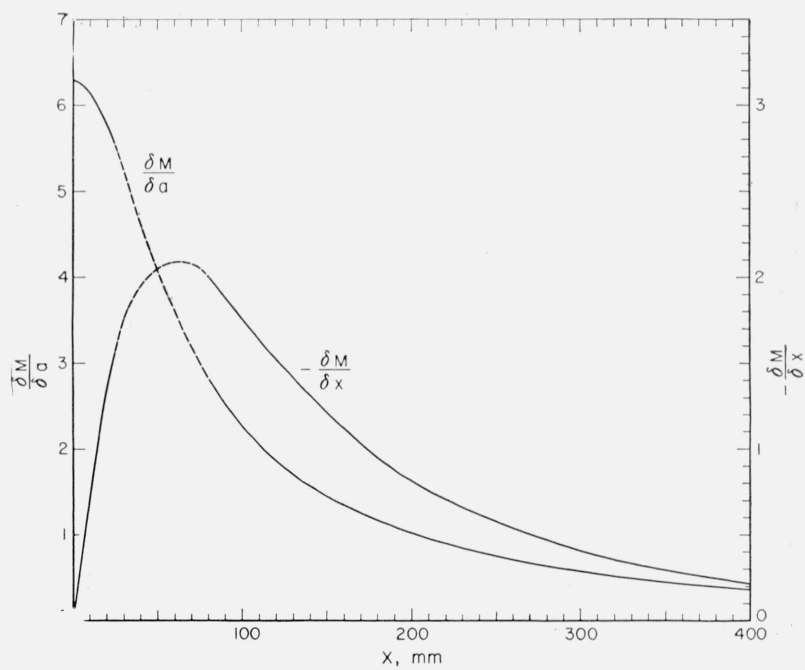

Figure 25. Partial derivatives $\delta M / \delta a$ and $\delta M / \delta x$ for primary and secondary circles as a function of axial separation.

Values of $\delta M / \delta a$ and $\delta M / \delta x$ in cgsm units of inductance per $\mathrm{mm}$.

in terms of the complimentary modulus $k^{\prime}=r_{2} / r_{1}$, using the rapidly convergent series given in eq 3 of Rosa and Grover's [16] tables. For values of $x$ between 81 and $399, \mathrm{~K}$ and $\mathrm{E}$ were taken from table XII of the same publication. Using diameter-variations, $\Delta D$, from the data of figure 11 , and values of $\partial M / \partial a$ from figure 25 , local corrections 
to $M$ were calculated by using the relation $\Delta M=f_{a}(\partial M / \partial a) \Delta D$, where $f_{a}$ is a numerical factor that takes the number of turns and units into account. The value of $\Delta M$ was calculated for 53 groups of three turns in each long section of the primary, and for each turn of the 25-turn middle section. These calculations were applied to both the December 1947 and the June 1948 diameter observations. To the nearest cgsm unit of inductance the total correction was the same, namely, +62. Expressed in proportional parts of $218 M_{0}$, this correction for irregularities in primary diameter is $+5.7 \mathrm{ppm}$.

TABLE 11. Data for calculating pitch correction

\begin{tabular}{|c|c|c|c|c|}
\hline$s_{n}$ & $\bar{c}$ & $x$ & $\frac{\partial M}{\partial x}$ & $\Delta M$ \\
\hline $\mathrm{cm}$ & microns & $m m$ & & \\
\hline 76 & -0.4 & 380 & -0.24 & +0.4 \\
\hline 68 & +.5 & 340 & -.31 & -.7 \\
\hline 60 & -2.6 & 300 & -.41 & +4.6 \\
\hline 52 & -3.8 & 260 & -.54 & +8.9 \\
\hline 44 & +.2 & 220 & -.71 & -.6 \\
\hline 36 & +.8 & 180 & -.96 & -3.4 \\
\hline 28 & -1.6 & 140 & -1.30 & +9.1 \\
\hline \multirow[t]{2}{*}{20} & -2.0 & 100 & -1.76 & +14.9 \\
\hline & & & & +33.2 \\
\hline
\end{tabular}

The information required for calculating the correction for axial mislocation of turns is summarized in table 11 . The quantities $s_{n}$ and $\bar{c}$ are taken from table 2 . The values of $\partial M / \partial x$ are taken from the curve (fig. 25) for each value of $x$, where $x=s_{n} / 2$ in millimeters. The corrections to $M$ are calculated for each double group of 20 turns by using the relation $\Delta M=f_{x}(\partial M / \partial x) \bar{c}$, where again $f_{x}$ is a numerical factor that takes proper account of turns and units. The last posted $\Delta M$ value of +14.9 takes cognizance of the fact that pitch measurements at $s_{n}=20$ included in the group one turn too many, because there are 159 active turns in each long section of the primary instead of 8 times 20. Expressed in proportional parts of $218 \mathrm{M}_{0}$, the total correction for pitch variations in the two long sections of the primary is $+3.0 \mathrm{ppm}$.

The calculation in table 11 takes no account of possible variations in pitch in the central 25-turn section of the primary because no satisfactory means were available for measurements in this short section. However, in the range of $x$ from 0 to $25 \mathrm{~mm}$, $\partial M / \partial x$ is practically a linear function of $x$, and consequently it is quite easy to derive an analytical expression for the integral value of $\Delta M$ over the 25-turn section in terms of an assumed deviation in pitch. If, for example, it is assumed that the pitch of the central section of the primary differs from the average value $p_{o}$ by $10 \mathrm{ppm}$, it can be shown that the correction would amount to 0.8 cgsm unit of inductance, which can be neglected because its influence on the total pitch correction is less than $0.1 \mathrm{ppm}$. Because of the method employed in lapping the continuous groove in the porcelain form, it appears unlikely that pitch variations exceeding $10 \mathrm{ppm}$ could exist.

6. Correction for eccentricity. Chester Snow has provided a formula, remarkably simple in form, by means of which a correction for eccentricity of primary turns may be calculated. Consider two coaxial parallel circles. If one of the circles is displaced in its own plane so that its center departs a small distance, $e$, from the axis of the other, the change in $M$, for small values of $e$, is given by the equation

$$
\Delta M=-\frac{e^{2}}{4} \frac{\partial^{2} M(x)}{\partial x^{2}},
$$

where $M(x)$ is Maxwell's expression for the mutual inductance of coaxial circles.

The equation for $\Delta M$ is independent of the azimuth of the radius vector whose length is $e$. The exact expression for $\partial^{2} M(x) / \partial x^{2}$ may readily be obtained by performing the indicated differentiation, but for present purposes it is adequate to differentiate graphically the $\partial M / \partial x$ curve shown in figure 25. The results are plotted as the curve of figure 26.

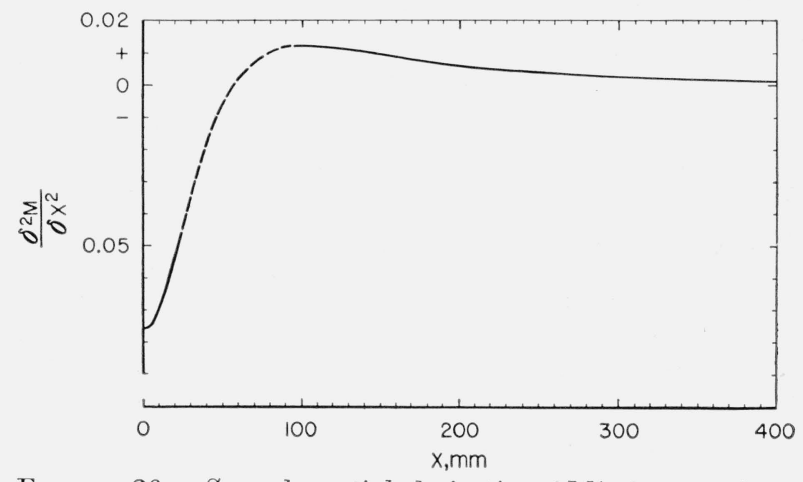

Figure 26. Second partial derivative $\delta^{2} M / \delta x^{2}$, as a function of axial separation $x$, for primary and secondary circles.

Values of $\delta^{2} M / \delta x^{2}$ in cgsm units of inductance per $\mathrm{mm}^{2}$.

From straightness measurements described in a previous section of this paper, it was determined that no group of turns in the long top and bottom 
sections of the primary was off-center by more than $10 \mu$ with respect to the mean location of the central 25 turns of the primary. If it is assumed that 8 groups of 20 turns each in the top section and 8 groups of 20 turns each in the bottom section of the primary are all off-center by this amount, the total change in $M$ is

$$
\Delta M=-20 N_{s} \frac{e^{2}}{4} 2 \sum_{1}^{8}\left(\frac{\partial^{2} M}{\partial x^{2}}\right)_{x_{j}}
$$

where $N_{s}$ is the number of secondary turns, 218, and $x_{j}$ is the axial distance to the center of each of the 8 groups of primary turns. Taking the required values of $\left(\partial^{2} M / \partial x^{2}\right)$ from figure 26 and making the indicated summation, the value of $\Delta M$ is found to be $-0.0097 \mathrm{cgsm}$ unit of inductance, an amount which is entirely negligible.

Equation 43 may be used to evaluate the effect of a displacement of the whole primary with respect to the secondary, i. e., an estimate of the effect of a lateral displacement of the secondary may be made. It will be noted that the sign of $\Delta M$ will reverse when the sign of $\partial^{2} M / \partial x^{2}$ reverses. From figure 26 it is evident that $\partial^{2} M / \partial x^{2}$ is positive in the regions where the long top and bottom sections of the primary are located, whereas $\partial^{2} M / \partial x^{2}$ is negative in the region occupied by the central section of the primary. As a result, displacement of the secondary from the coaxial position causes $M$ to increase with respect to the central section of the primary and to decrease with respect to the remainder of the primary. The two effects tend to compensate, rendering the inductor relatively insensitive to lateral displacement of the secondary. If the secondary is displaced $0.5 \mathrm{~mm}$ and $e$ therefore taken as 0.5 in eq $43, \Delta M$ with respect to the central section of the primary is +22.0 , whereas the total $\Delta M$ with respect to the top and bottom sections is -24.3 , a net change of -2.2 cgsm units of inductance, or $-0.2 \mathrm{ppm}$ when expressed in proportional parts of the total inductance. This is in accord with experimental results. It has been observed that a secondary displacement of several tenths of a millimeter produces no measurable change in the balance point during electrical measurements.

From the results of this portion of the investigation it has been established that any lack of straightness in the primary is quite negligible. In fact, the question may safely be disregarded unless the primary is so crooked that it is obvious to the naked eye using a good straightedge. Heretofore it has been assumed that no appreciable errors were introduced due to lack of straightness in the primary of inductors of this type.

\section{(c) Effective Value of $M$}

The effective value of $M$ may now be calculated using the corrections obtained above. The data required are summarized as follows:

Correction for secondary distribution, $c_{s-\ldots}$

$\Delta M$ due to three extra turns _............

Correction for primary current distribution

Correction for primary wire distortion _.....

Correction for primary diameter variation _

Correction for primary pitch variation _...

Total primary correction, $c_{p} \ldots \ldots \ldots \ldots$

$-75.8 \mathrm{ppm}$
149,944
$-12.9 \mathrm{ppm}$
$+0.5 \mathrm{ppm}$
$+5.7 \mathrm{ppm}$
$+3.0 \mathrm{ppm}$
$-3.7 \mathrm{ppm}$

Applying eq 20, it is found that

$M=[215 \times 49991.32(1-0.0000758)+149944]$

$$
(1-0.0000037)=10,897,220 \text {. }
$$

To the nearest $1 \mathrm{ppm}$, the effective value of $M$ at $23.0^{\circ} \mathrm{C}$, as of March 1948 , was therefore

\section{$M=10,897,220 \mathrm{cgsm}$ units of inductance.}

\section{Speed Control}

\section{General Considerations}

The average frequency of reversal of the primary current and of the associated switching operations is maintained constant within 1 or 2 parts in $10^{7}$ by means of an electronic speed-control system. The commutators, reversing generator for the primary current, inductor generator for the secondary circuit, two special rotary switches, and a d-c generator are mounted on a common shaft, to which is belted a d-c driving motor.

The method of control is to set the driving motor to turn the shaft at a speed somewhat higher than is required, and then to reduce the speed by throwing a load onto the generator. The load is so chosen that, if applied continuously, the speed of shaft rotation will be too low. By rapidly throwing this load on and off, and properly proportioning the on and off periods, it is possible to maintain the average speed at the proper value. Variations in friction loads on the rotating system can thus be compensated by corresponding changes in the average load on the generator.

The speed of the shaft is synchronized by means of a 1,000-c/s emf obtained from the Central Radio Propagation Laboratory of this Bureau. 
This frequency is obtained by stepping down electrically from a $100,000-\mathrm{c} / \mathrm{s}$ frequency produced by crystal oscillators. The $1,000-\mathrm{c} / \mathrm{s}$ frequency is as constant as the higher frequency, which is believed to be constant and equal to its nominal value to 1 part in 10 million or better. Before introduction into the speed-control circuit, this $1,000-\mathrm{c} / \mathrm{s}$ signal is filtered to improve the wavèform and amplified to an effective value of $40 \mathrm{v}$.

To obtain synchronization, a contact on the rotating shaft functions briefly four times per revolution. This momentarily connects the $1,000-$ cycle emf to the grid of a thyratron. If, at the instant of application, the 1,000-cycle emf has a value above the grid potential at which the tube will fire, the tube becomes conducting and a load is thrown onto the generator, slowing down the rotating shaft and causing it to drop back in phase. If the contact is made at a time when the emf is below the firing potential of the tube, it remains nonconducting and the shaft continues to advance in phase because of the excess power supplied by the motor. Just before each application of the 1-000-cycle emf, the plate circuit of the thyratron is opened mechanically for an instant to restore the tube to its nonconducting condition, if necessary. Changes in generator load cause the rotating shaft to "hunt" about that phase of the control frequency at which the emf equals the firing potential of the tube. This hunting is at the rate of 4 or 5 oscillations per second, but the speed of the shaft, when averaged ${ }^{3}$ over sufficiently long periods of time, is as constant as the frequency of the controlling emf.

A system of speed control of this type is not inherently stable, because the torques that restore the drive shaft to the proper phase do not stop the shaft at the correct phase but throw it out of phase in the opposite sense. Because of time lags in applying and removing the torques, an oscillation may be started that would build up in amplitude, unless some method of damping the oscillations were provided. To some degree electromagnetic damping occurs in the generator and driving motor.

In order to assure stable operation, even in the absence of adequate damping, the load on the generator is thrown on or off in steps. Suppose the shaft has advanced in phase and the mercury-

${ }^{3}$ For further discussion, see section IX. vapor tube has tripped and a load is thrown on the generator. This load provides a torque that stops the advance in phase and then produces a backward shift of the phase. The electrical circuit is so arranged that the torque is initially large but quickly diminishes in magnitude. This means that a large torque is available to stop the advance in phase; but a much smaller torque retards the phase toward the correct position, and consequently the "overshoot" in phase is small. The circuit arrangement is such that the change in torque is applied in steps whether the shaft is being either advanced or retarded in phase. The variable torques are secured by means of an inductor placed in the armature circuit of the generator.

The action of the inductor may be understood by the use of the schematic circuit shown in figure 27. In this figure, $E$ is the battery supplying the

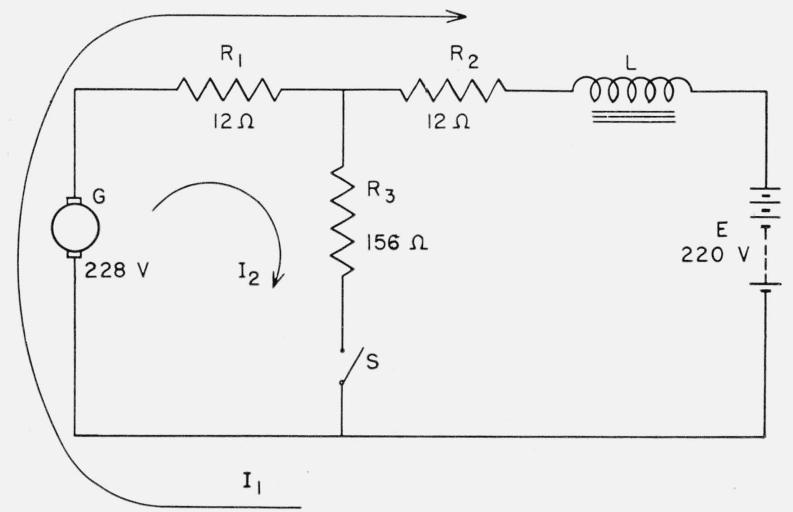

Figure 27. Elementary circuit diagram of loading generator.

driving motor and the field windings of generator $G$, which is excited so as to give an emf across its armature terminals of about $228 \mathrm{v} ; R_{1}$ and $R_{2}$ are current-limiting resistors; and $R_{3}$ is the resistance load connected across the generator terminals when switch $S$ is closed (i. e., the thyratron is ionized).

Discussing this equivalent circuit by the use of Maxwell's mesh currents for the two positions of switch $S$, we obtain the following results.

\section{(a) Switch $S$ Opened}

For steady state conditions, $I_{2}$ is zero, and $I_{1}$, the current drawn from the generator armature, $G$, is $8 / 24$, or 0.33 amp. Consequently, this load 
is $(0.33) \times 228=75 \mathrm{w}$, which is, of course, being supplied by the driving motor.

\section{(b) Switch $S$ Closed}

At the instant the switch $S$ is closed, the inductive action of the inductor tends to prevent the current $I_{1}$ from changing. However, if there is no inductance in circuit 2 , the current $I_{2}$ from $G$ increases from zero to $224 / 168$, or approximately 1.33 amp. Then the total current drawn from $G$ is $1.33+0.33=1.66 \mathrm{amp}$. At this time the load is $228 \times 1.66=378 \mathrm{w}$. A few hundredths of a second later the direction of the current, $I_{1}$, is reversed, and the current drawn from $G$ is reduced to approximately $1 \mathrm{amp}$, and thus the load is reduced to about $233 \mathrm{w}$.

\section{(c) Switch $S$ Opened}

At the instant switch $S$ is opened, $I_{2}$ becomes zero, and a change in the magnitude or direction of $I_{1}$ is opposed by the inductance of $L$. As $I_{1}$ has a magnitude of $0.36 \mathrm{amp}$ and is producing motor action, $G$ supplies approximately $82 \mathrm{w}$ to the shaft. However, in a few hundredths of a second, current $I_{1}$ reverses in direction and returns to the steady condition described in (a).
The above calculations are based on the assumption that the inductance of circuit 2 is negligible in comparison with inductor $L$. Measurements show that the inductance of circuit 2 is not much greater than $0.1 \mathrm{~h}$, whereas that of $L$ is about 1 $\mathrm{h}$, even with a direct current of $0.4 \mathrm{amp}$ flowing through it.

\section{Control Circuit in Detail}

The circuit arrangement for speed control is shown in figure 28 . In this figure, $D$ and $F$ represent rotary switches mounted on the shaft whose speed is to be controlled, and $M$ represents the d-c motor that drives the shaft and supplies power in excess of the mechanical load. The shaft is connected directly to the control generator, $G$, which absorbs power intermittently from the rotating shaft,

The thyratron, $T$, is a General Electric mercuryvapor tube F. G. 57, the grid of which is negatively biased by the battery $B$. Four times each revolution of the shaft, the time pick-up switch, $D$, momentarily removes the biasing battery, $B$, and allows the $1,000-\mathrm{c} / \mathrm{s}$ control emf to be impressed on the grid of the thyratron. If the rotating mechan-

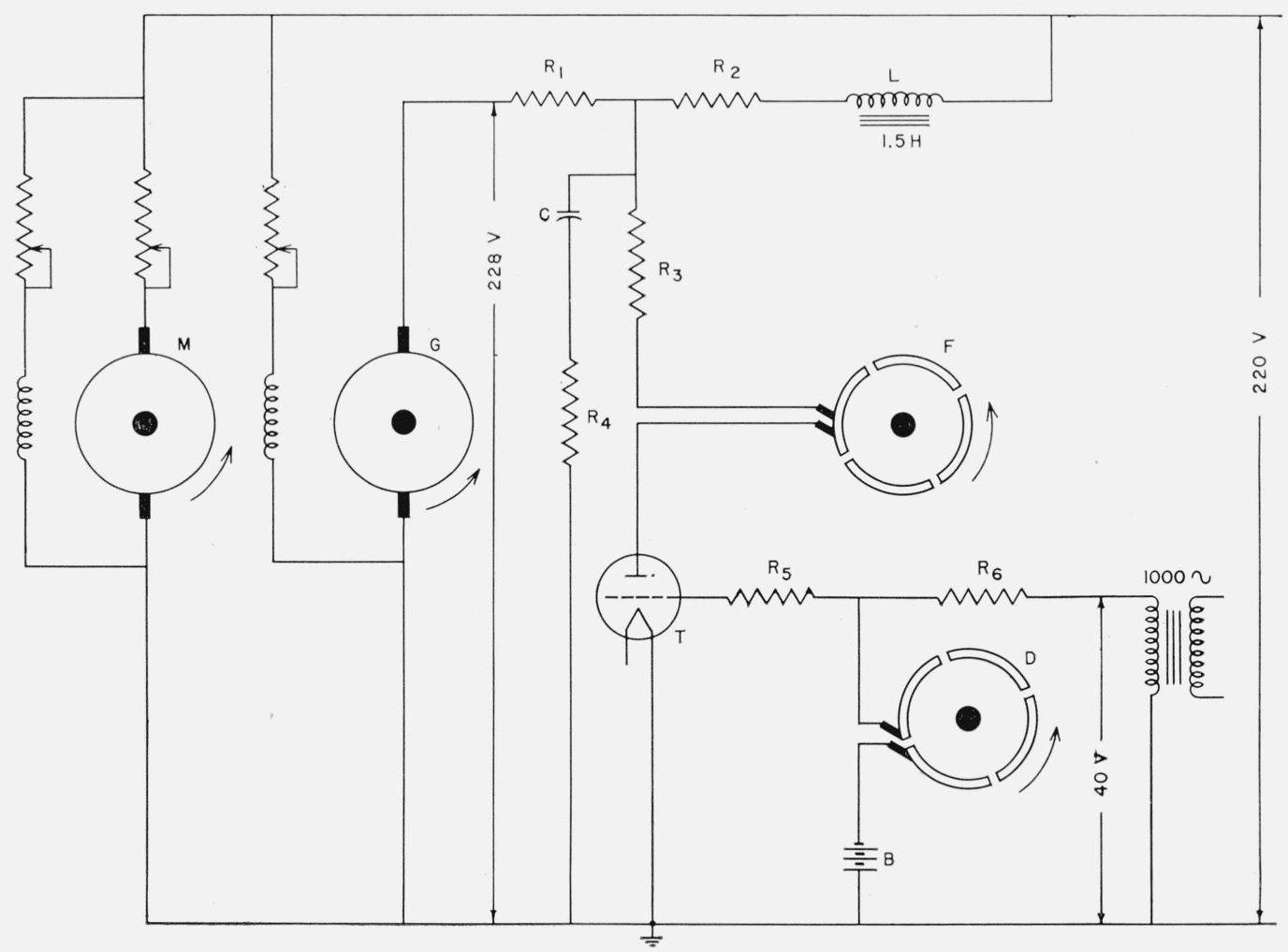

Figure 28. Partial schematic circuit diagram of speed-control apparatus. 
ical system is slightly ahead of its schedule as determined by the phase of the 1,000-c/s emf, the control emf is of sufficient magnitude to trip (ionize) the thyratron. The thyratron, when ionized, completes the circuit through resistance $R_{3}$, and this resistance load on the generator slows down the entire rotating system. The switch, $F$, breaks the plate circuit through the mercury-vapor tube each quarter of a revolution of the shaft for approximately $0.001 \mathrm{sec}$, the time required to deionize the tube.

Figure 29 shows the mechanical connections of

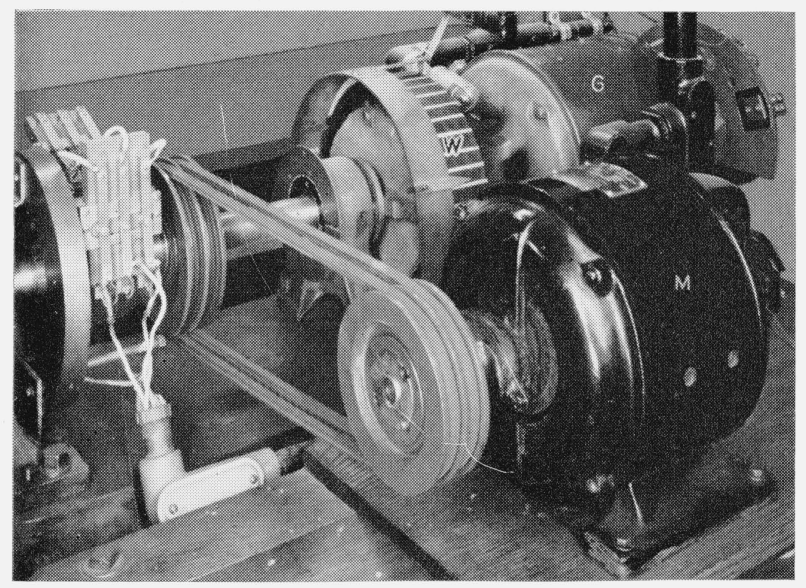

Figure 29. Motor drive for constant-speed shaft.

the speed-control equipment. In this figure, $G$ is a 2-hp, 1,135-rpm, 220-v shunt wound d-c motor, used primarily as a generator, connected directly to the shaft of the reversing commutators by a stiff leather coupling disk. The $\mathrm{d}-\mathrm{c}$ motor, $M$, is $1 \frac{1}{2} \mathrm{hp}, 1,725-\mathrm{rpm}, 230-\mathrm{v}$ shunt wound, and it supplies the power to drive the shaft at approximately 1,360 rpm. The mechanical loads on the shaft are approximately $500 \mathrm{w}$. These power losses are supplied by this motor, which is belted to the shaft with the proper pulley ratio. The power supplied to motor $M$ can be controlled by adjustable resistors in series with its armature and field windings. The flywheel, $W$, on the constantspeed shaft has a moment of inertia of approximately one million g-cm. ${ }^{2}$

The speed of the shaft is such that it makes 1 revolution in $0.044 \mathrm{sec}$. When starting, the speed of the shaft is manually held close to this value for a few seconds. This is accomplished by manually adjusting the speed of the driving motor and observing the speed of the shaft by means of a stroboscope consisting of lines on flywheel $W$ and a small neon lamp flashed by the 1,000-c/s emf. The automatic speed-control device is then connected into the circuit and begins to operate.

Referring again to figure 28 , it is seen that when switch $D$ closes the circuit through battery $B$, the potential applied to the grid of the thyratron is that furnished by battery $B$. This battery was so selected that for the plate voltage used with the thyratron, the grid potential is several volts less than the tripping potential. Four times during each revolution of the shaft, the switch $D$ removes the fixed biasing potential by opening the circuit and allows the $1,000 \mathrm{c} / \mathrm{s}$ emf to be applied to the grid. If the shaft is ahead of schedule, this grid voltage exceeds the tripping potential and the tube ionizes and conducts current, thus closing the circuit through resistor $R_{3}$. The manner in which the potential on the grid of the mercury-vapor tube varies during a small fraction of a revolution of the shaft when it is ahead of schedule, is shown in the upper part of figure 30 . In this figure the grid potential, $E_{g}$, is shown to be less than the tripping potential until a time $T_{1}$, when the commutator opens the circuit of the battery and allows the $1,000 \mathrm{c} / \mathrm{s}$ emf from the crystal oscillator to be impressed on the grid of the thyratron. For the case shown, the rotating mechanical system is a few hundred-thousandths of a second in advance of the synchronous position. Consequently, the control voltage exceeds the tripping voltage when it is impressed on the grid of the tube at time $T_{1}$. This results in the tube's closing the circuit, thereby connecting an electrical load to the terminals of the control generator, which causes the generator to slow down, thus reducing the speed of the shaft. After approximately $0.01 \mathrm{sec}$, the plate circuit is opened by $F$ (fig. 28) thus removing the electrical load from the generator. Approximately 0.001 sec later, i. e., after the tube has deionized, switch $F$ recloses the plate circuit, and then switch $D$ opens the circuit of battery $B$, and again allows the control voltage to be impressed on the grid of the thyratron. If at this time the moving mechanical system is still slightly ahead in time displacement, the thyratron again trips and the same procedure is repeated. However, if the mechanical system is now slightly behind the electrical signals, the voltage impressed on the grid circuit is less than the tripping voltage, as shown in the bottom part of figure 30, and the thyratron remains noncon- 


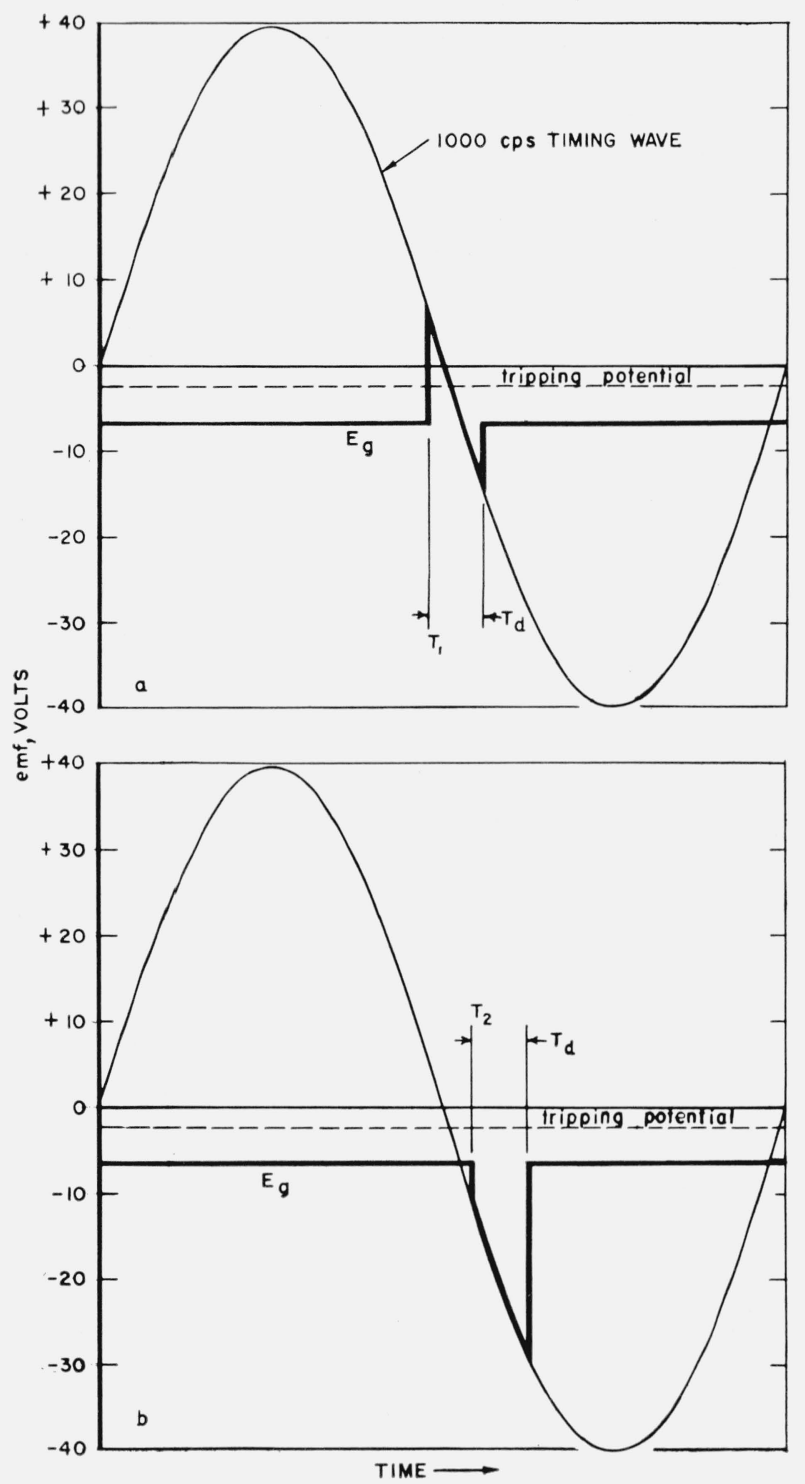

FIGURE 30. Variation of grid emf during a time signal pick-up interval, for two different phase angles.

ducting. Adjustments are made such that the electrical load is on approximately one-half the time. This means that in operation the shaft is alternately ahead and behind the timing device. The period of oscillation is approximately 0.25 sec, and the amplitude is some $20^{\circ}$ or $30^{\circ}$ of 1 cycle of the 1,000-cycle emf. The top portion of figure 30 is idealized to the extent that the grid potential, $E_{g}$, may depart from the curve as shown during the brief time interval $T_{d}$, when current flowing in the grid circuit of the tube distorts the timing wave. This does not affect the operation of the circuit, however.
After the apparatus has been in operation for a sufficient time for the control rheostats and the field windings of the motor and generator to come to a steady temperature, the constant-speed shaft will run for hours with little attention. Before using the measuring circuit, however, it is desirable to set the controls for the position where the amplitude of hunting is a minimum. Since the shaft makes 1 revolution every $44 \mathrm{~ms}$, the speed of rotation is $1,000 / 44 \mathrm{rps}$, and this is the value of $n$ to be used in eq 3. The average value of $n$ is $1,000 / 44$ to the same accuracy as that of the 1,000-cycle controlling emf, and this is believed to be correct to 1 or 2 parts in $10^{7}$.

\section{The Measuring Circuit}

\section{General Considerations}

As explained in connection with figure 1, a direct current, 1 , is sent through the standard resistor, $R$, and the primary winding of the mutual inductor, $M$. The measuring procedure requires that the current through the winding of the mutual inductor be reversed about 45 times a second without affecting the current through the resistor. As the desired accuracy of balance is at least one part in a million, with about 1 amp flowing, the above requirement appears to be very difficult to meet, since in order to reverse connections to the primary of $M$ it is necessary either to short or to open the primary circuit.

The better method is probably to short-circuit the connections to the primary winding and to reverse the connections while they are shorted. It was first planned that the primary battery would be of high voltage with a correspondingly large series resistance, so that the percentage change in resistance would be small when the primary of the inductor was shorted. With a very high inductance in the circuit the change in current might be kept small. However, it was decided that an easier solution of the problem would be to arrange the circuit so that the two terminals of the primary reversing switch, at the point of shorting, would be always at the same potential, in which case no change in the current would result from the short-circuit.

That a portion of a circuit can be short-circuited without affecting the value of current in the remainder can be seen from figure 31 . Suppose 


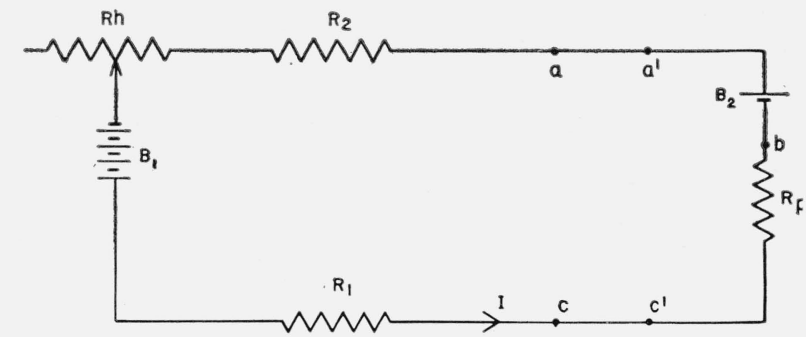

FIgURE 31. Diagram illustrating how two points in a current-carrying circuit may be brought to the same potential.

batteries $B_{1}$ and $B_{2}$ are sending a current, $I$, through resistors $R_{1}, R_{2}$, and $R_{p}$. By properly adjusting the current, the potential difference across $R_{p}$ can be made to be exactly the same as the emf of battery $B_{2}$. Neglecting the internal resistance of $B_{2}$, or regarding it as part of $R_{p}$, the potential difference from $b$ to $c$ will then be the same in value but opposite in direction to the emf between the points $a$ and $b$. The two points $a$ and $c$ are then at the same potential, and a short-circuit from $a$ to $c$ will not affect the current flow. If, after shorting, the circuit through $B_{2}$ and $R_{p}$ is opened by disconnecting at $a^{\prime}$ and $c^{\prime}$, the current through $R_{1}$ and $R_{2}$ will flow across the short-circuit without any change in value. It is possible then to reverse connections to the battery $B_{2}$, and also to connect $a^{\prime}$ to $c$ and $c^{\prime}$ to $a$, after which the current will flow through $R_{p}$ in a direction opposite to that of the original current, and the current in the shortcircuit will drop to zero. The short-circuit can now be removed without disturbance to the current. By this cycle of operations it is theoretically possible to reverse the current through $R_{p}$ without affecting the value of the current in the part of the circuit to the left of $a c$. Practically, it can be accomplished only approximately, because the resistance of the short-circuit can never be zero.

In the above procedure, no disturbance will be caused in the part of the circuit to the left of $a$ and $c$ if the connections and current in the circuit to the right are reversed in such a way that the current in the two parts are the same when the short is applied or removed. As long as this condition is met, the method of reversal of the right circuit is of no consequence. The connections to the battery may be interchanged by means of a simple reversing switch, or a special switch may be used to make the reversal in steps. The presence of inductance in this part of the circuit might affect the way in which the current reverses but not the final result if the currents match. If the inductance were large, it would be necessary to delay removal of the short-circuit across $a$ and $c$ until any transient effect of the inductance had disappeared. Subject to this limitation, the above method for reversing the current through resistor $R_{p}$ could be followed for reversing the current through the primary of a mutual inductor without disturbing the direct current flowing through the remainder of the circuit.

For our particular problem of reversing the current through the primary of a mutual inductor, it is desirable to control the wave form of the current through the inductor during reversal, in order to control the wave form of the induced secondary emf. It was therefore necessary to build a special type of reversing apparatus for controlling the change of the current during its reversal. This will be called a "reversing generator," although it is in reality a battery and a connected commutator and resistance network from which a reversing emf of the desired wave form can be obtained. Its function is to maintain a zero potential difference across the primary shorting switch and at the same time reverse the current beyond this switch in a predetermined manner. In order to better understand its operation, and its relation to the shorting switch, let us consider the operation of the primary circuit as a whole.

\section{The Circuit as a Whole}

The essential features of the measuring circuit are shown schematically in figure 32 . The current, $I$, in the primary circuit is furnished by $B_{1}$ a 10 -cell automobile storage battery. The circuit was designed for a primary current of 1 amp. but can be balanced with other currents, as the balance is independent of the magnitude of the primary current. The current is adjusted in value by means of an ordinary tubular rheostat, $R h$.

The primary current passes through $K_{p}$, an iron-cored choke coil. The iron core contains an air gap, and the choke has an inductance of about 6 henries when carrying a current of $1 \mathrm{amp}$. In addition to the iron-cored choke, the two "electronic chokes," $V_{1}, V_{2}$, [5], are used to help maintain a constant primary current. These electronic chokes are amplifiers with a 1:1 amplification ratio and with the output voltage $180^{\circ}$ out of phase with the input voltage. Thus a small poten- 


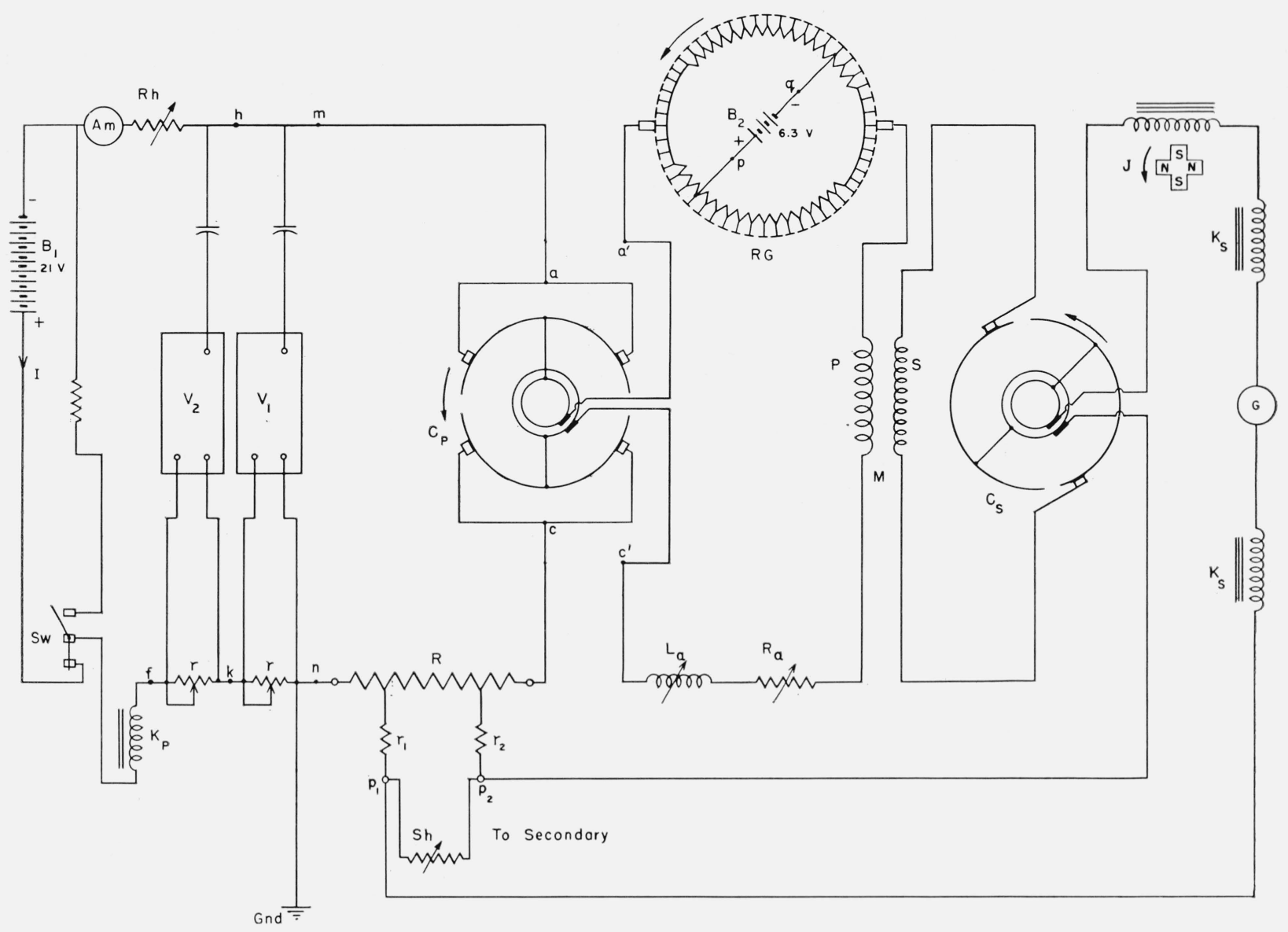

Figure 32. Partial diagram of the measuring circuit.

tial difference arising across the input circuit of either choke will be neutralized, as far as current in the primary circuit is concerned, by an equal potential difference, which will be produced across the terminals of its plate resistor, $r$. Two such chokes are used in cascade and together they will neutralize, within about 0.2 percent, any sudden potential difference, not exceeding $0.1 \mathrm{v}$, which might appear across the primary reversing switch, $C_{p}$, because of imperfect operation of the other components of the circuit.

Beyond the electronic chokes the primary current flows through resistor $R$, which is to be measured. This is a four-terminal 1-ohm resistor of the highest quality, with an adjustable shunt of known value, which is used for balancing the measuring circuit. Beyond this resistor is located rotary switch $C_{p}$, which is used to short circuit the primary circuit from $a$ to $c$ while the current is reversed in that part of the primary circuit to the right. The reversing generator, $R G$, controls the current as it is reversed in this part of the circuit, and hence through the primary of the mutual inductor, $M$. The adjustable resistor and inductor, $R_{a}$ and $L_{a}$, are included and adjusted to make the resistance and inductance equal to those which were assumed, for the circuit to the right of the shorting switch, in designing the reversing generator. The resistance and inductance of $R_{a}$ and $L_{a}$ need not be known, as the balance of the circuit does not depend upon the resistance or self-inductance of this part of the circuit. Their use improves the matching of the current in the two parts of the primary circuit and thus reduces voltage disturbances that might appear across shorting switch $C_{p}$.

The emf induced in the secondary of the mutual inductor by the reversing primary current is rectified by means of reversing switch $C_{s}$. This reversing switch is of a simple rotary type, having copper segments and copper-graphite brushes. In addition to the emf rectified by $C_{s}$, and the 
steady potential difference across $R$ caused by the primary current, there is in the secondary circuit a square-topped emf wave induced by the inductor generator, $J$. This inductor generator has four poles, as shown, which are energized through slip rings by a direct current. The sum of the three emf's is impressed upon the d-c galvanometer, $G$, in series with which are placed two large choke coils, $K_{s}$, which limit the a-c component of the current through the galvanometer.

The cycle of operation of the circuit is as follows:

With switch $C_{p}$ in the series position, as shown, the test current of about 1 amp flows through resistor $R$ and through primary $P$ of mutual inductor $M$. After having been connected in this position for about one-sixth of the cycle, a short is connected across the circuit by $C_{p}$, which is maintained until generator $R G$ has reversed the current through the primary of $M$. This reversal requires about two-sixths of the cycle, after which switch $C_{p}$ makes a series connection but with leads interchanged. Current $I$ is then allowed to flow in the reverse direction through the primary winding of $M$ for one-sixth cycle, after which it is again reversed to the starting direction, this second reversal requiring the remaining twosixths cycle. It will be noticed that after each reversal, the circuit is series-connected for about one-sixth cycle. During these times the reversing generator is producing a constant emf of such a value that it would maintain a current equal to $I$ through the primary of the inductor if the short circuit had remained. If the emf of the reversing generator is not exactly correct, the tremendous equivalent inductance in the remainder of the primary circuit will nevertheless bring the current
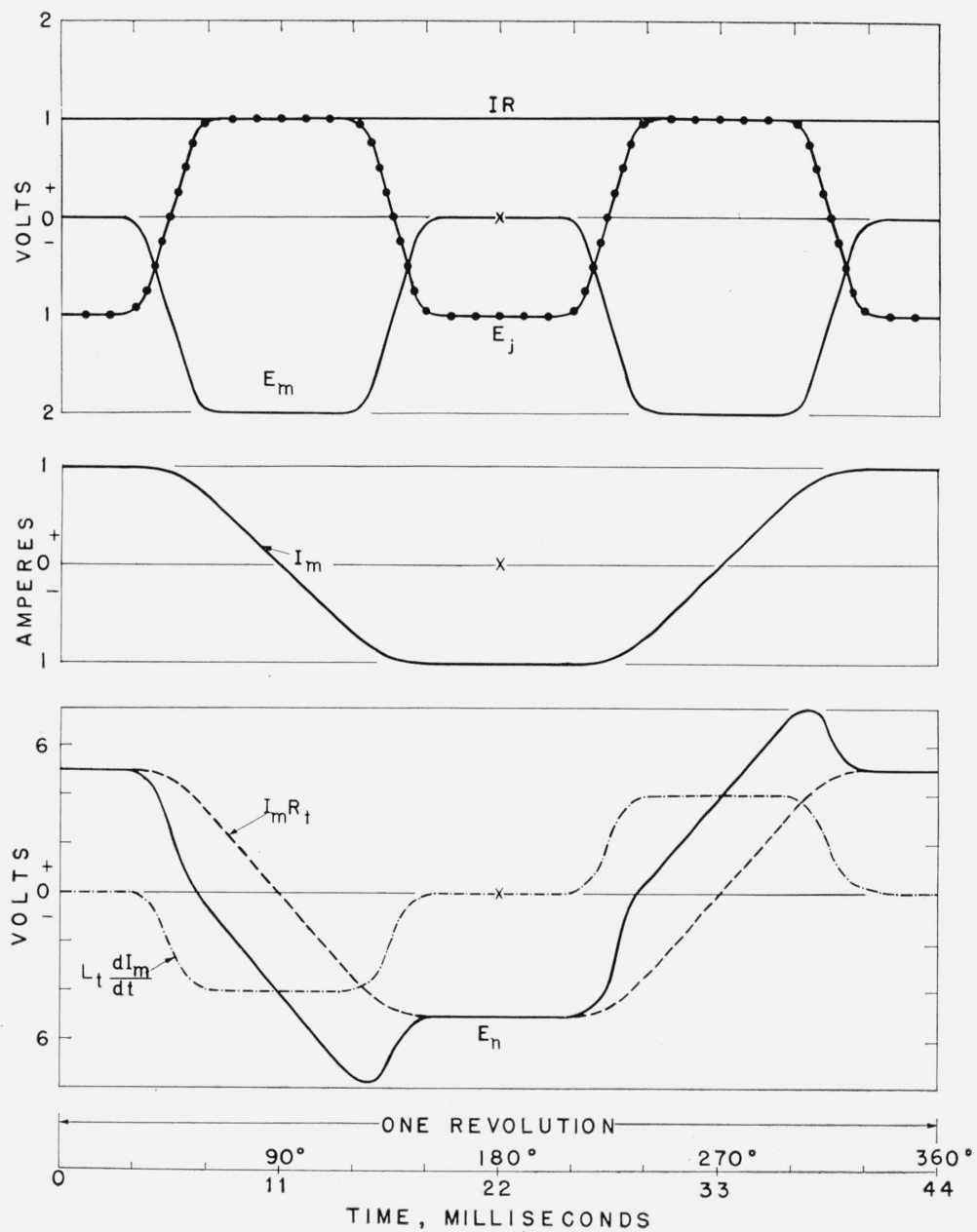

FIGURE 33. Plot of variation of certain currents and voltages during one revolution of the drive shaft.

Top: voltages appearing in galvanometer circuit. Center: current in primary of mutual inductor. Bottom: voltage at terminals of reversing generator. 
to the value $I$ so quickly that a constant current will flow through the primary of the inductor for a large portion of this one-sixth cycle.

During the time in which the current in the primary is constant, the secondary induced emf is zero, and during this part of the cycle the secondary leads are reversed. Before this reversal the current through the primary winding of the inductor has attained practically a d-c distribution over the cross section of the wire. This is the distribution for which the inductance is calculated, and no correction is required for the effect of frequency upon current distribution.

The cycle of operation is shown graphically in figure 33. In the upper part of the figure are shown the emf's in the galvanometer circuit. Here $E_{j}$ is the emf introduced in the circuit by the inductor generator, which from its nature averages zero. The ordinates of this curve were measured with the generator in actual operation. The straight line $I R$ is the potential drop across resistor $R$. The third curve, $E_{m}$, is the emf which must be induced in the secondary of the mutual inductor by the reversing primary current to balance at every instant the algebraic sum of the other two emf's in the galvanometer circuit.

From the third curve the data for the curve $I_{m}$ of the central part of figure 33 were calculated. This shows the way in which the current must vary in the primary of the mutual inductor to generate the emf $E_{m}$, assuming that the reversal of the secondary connection is made at the time marked $X$. It should be noted that the frequency of the cycle of $I_{m}$ in the primary of the mutual inductor is only half that of the frequency of the rectified emf in the secondary circuit.

The curve $I_{m} R_{t}$ at the bottom of figure 33 was then calculated by multiplying $I_{m}$ by the total resistance, $R_{t}$, of the part of the circuit to the right of the shorting commutator, $C_{p}$, figure 32 , exclusive of the reversing generator. From the rate of change of $I_{m}$ and the total self-inductance, $L_{t}$, of this same part of the circuit, the induced emf, $L_{t} d I_{m} / d t$, was calculated for the entire cycle. The sum of the two emf's, $I_{m} R_{t}$ and $L_{t} d I_{m} / d t$, i. e., $E_{n}$, is the emf that must be produced by the current-reversing generator in order to reverse the primary current as shown by the curve for $I_{m}$, thus inducing in the secondary the emf $E_{m}$.

\section{Details of Certain Components}

(a) The Reversing Generator

The energy supplied to that part of the primary circuit to the right of the shorting switch, $C_{p}$, (fig. 32) is furnished by the reversing generator. This generator was designed to take the current in the primary of the mutual inductor through the current cycle shown in figure 33, assuming a short-circuit maintained across $C_{p}$. The removal of the short-circuit brings the primary current through the inductor to the same value as that through the resistor under test at the time of reversal of the secondary connections, thus correcting for an imperfect operation of the reversing generator.

The reversing generator and its attached circuit are shown schematically in figure 34 . The storage battery, $B_{2}$, is connected to two parallel resistance circuits, each having a resistance $A$. In effect, this entire circuit rotates with reference to the contacts $b$ and $b^{\prime}$, and the potential difference

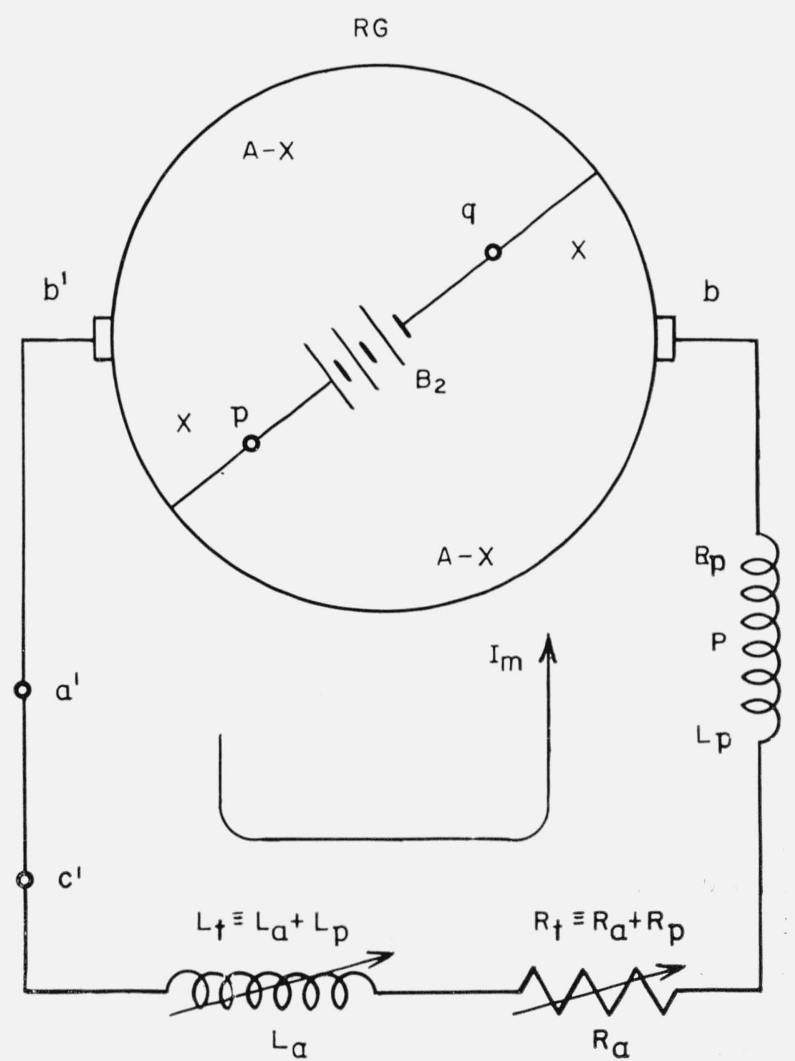

FIgURE 34. Schematic diagram for reversing-generator oircuit. 
across these contacts is connected in series with the primary of the mutual inductor. During rotation, the resistance, $X$, must vary in such a way that the potential difference between $b$ and $b^{\prime}$ will vary in accordance with curve $E_{n}$ of figure 33 , while the current from $b^{\prime}$ and back into $b$ is, at corresponding parts of the cycle, shown by curve $I_{m}$ of the same figure.

The design of the reversing gen rator required the calculation of $X$, the resistance between the brush contacts $b$ and $b^{\prime}$ and the points of attachment of the storage battery, for all parts of the cycle. To calculate $X$, use is made of Thévenin's Theorem. In applying his theorem, a calculation is made of the emf, $E$, which would appear between $a^{\prime}$ and $c^{\prime}$ if the circuit were opened between them, and then the current is calculated on the assumption that this emf is applied in the reverse sense to $a^{\prime} c^{\prime}$, and any other emf is reduced to zero.

For present purposes it is sufficiently accurate to neglect the resistance in battery $B_{2}$, whose emf will also be called $B_{2}$. Then by inspection

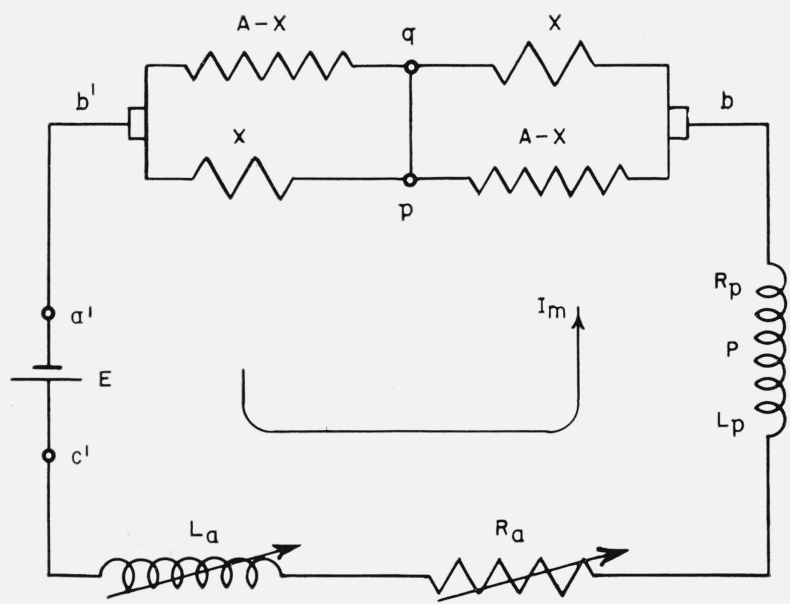

Figure 35. Application of Thévenin's theorem to reversinggenerator circuit.

$$
E=B_{2}\left(\frac{A-X}{A}-\frac{X}{A}\right)=B_{2}\left(\frac{A-2 X}{A}\right) .
$$

If now $B_{2}$ is reduced to zero and $-E$ is inserted between $a^{\prime}$ and $c^{\prime}$, the circuit will be as shown in figure 35, and by 'Thévenin's Theorem the currents will be the same as those of circuit 34 . Then by Kirchoff's Law

$$
\begin{aligned}
R_{t} I_{m}+L_{t} d I_{m} / d t+2 I_{m} \frac{X(A-X)}{A} & =\mathrm{E} \\
& =B_{2} \frac{(A-2 X)}{A},
\end{aligned}
$$

or

$$
2 I_{m} X^{2}-2\left(B_{2}+I_{m} A\right) X+\left(B_{2}-E_{n}\right) A=0,
$$

where

$$
E_{n} \equiv R_{t} I_{m}+L_{t} d I_{m} / d t
$$

The values of $X$ for all parts of the cycle were calculated by solving eq 46, using the corresponding values of $I_{m}$ and $E_{n}$, as shown in figure 33; they are plotted in figure 36 , together with the curves for $I_{m}$ and $E_{n}$. The value of resistance $A$

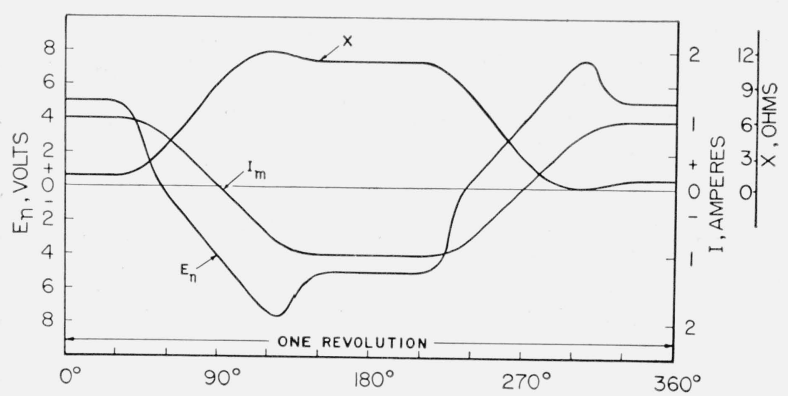

FIGURE 36. Data used in designing reversing generator.

is arbitrary, and it was taken as 12 ohms. The emf of battery $B_{2}$ must equal the maximum value of $E_{n}$, which in turn depends upon the resistance and inductance of the circuit in which the reversing generator operates. In actual use, $B_{2}$ may be changed to correspond to the emf of an integral number of battery cells, and the values obtained for $X$ from eq 46 will not be affected, provided $I_{m}$ and $E_{n}$ are changed in the same ratio as $B_{2}$. From eq 47 it is evident that $E_{n}$ is proportional to $I_{m}$ for given circuit parameters, whence it follows that for given values of $A, R_{t}$ and $L_{t}$ the solution for $X$ is unaffected by changes in $B_{2}$ if $I_{m}$ is changed proportionately, and this in turn means that the main primary current, $I$, must be adjusted accordingly.

To build the reversing generator of figure 34, a commutator was taken from a discarded generator and mounted on the constant-speed shaft that drives the reversing switches. This commutator has 177 segments, each $10 \mathrm{~cm}$ in length and 3.5 mm wide. Through a set of slip rings the storage battery is connected to points $180^{\circ}$ apart on the commutator. Brushes on the commutator for connecting to the external circuit are also spaced $180^{\circ}$. Pieces of resistance wire were soldered between adjacent segments of the commutator, the values of the resistance of each section being so chosen as to change the resistance between the brushes and the points of attachment of the battery leads in accordance with the $X$-curve of 
figure 36. The sections of resistance were not adjusted to a high accuracy, since at best the reversing of current in the primary winding is a step-by-step process, and an instantaneous match of the emf induced in the secondary and the remaining emf's in the secondary circuit cannot be attained. The irregularity caused by the step-bystep reversal is smoothed out to some extent by means of a $2-\mu$ f capacitor connected across the reversing generator.

This reversing generator was designed and built before the mutual inductor had been completed. The design assumed a primary current of $1 \mathrm{amp}$ and values of $5 \mathrm{ohms}$ and $22 \mathrm{mh}$ for $R_{\iota}$ and $L_{t}$, respectively. On this basis, the curve $E n$ of figure 33 was obtained, its maximum value being about $7.7 \mathrm{v}$. This meant that three or four leadacid cells should be used for $B_{2}$. Three cells were chosen because the lower level of primary current called for, about 0.8 amp, would produce less heat in various circuit components without materially reducing the sensitivity.

When the primary of the mutual inductor was finished it was found to have a resistance and self-inductance of $3.5 \mathrm{ohms}$ and $15 \mathrm{mh}$, respectively. The values for $R_{t}$ and $L_{t}$ were purposely assumed higher than those expected for the inductor, in order that the difference could be provided by adjustable circuit elements $R_{a}$ and $L_{a}$. The circuit could then be accurately set to the design values in order to obtain optimum performance.

(b) The Primary Reversing Switch

The construction of the primary reversing switch, which is of the rotary type, is shown schematically in figure 37. A 2-in. length of copper tubing $4 \mathrm{in}$. in diameter was split axially into two equal long and two equal short sections, and the sections were so mounted as to be insulated from one another. This part of the switch is driven by the constant-speed shaft of the measuring apparatus. The segments are represented by $S_{1}, S_{2}, S_{3}$, and $S_{4}$ of figure 37 . Riding on the split copper ring is a group of seven copper-graphite brushes attached to phosphor-bronze leaf springs. Three brushes, $B_{2}, B_{3}$, and $B_{4}$ are connected to one side of the primary circuit, and the four remaining brushes are connected to the other side. The two large copper segments, $S_{1}$ and $S_{3}$, are connected through slip rings (not shown) to reversing generator $R G$, the primary $P$ of the mutual inductor, the adjustable resistor $R_{a}$, and the small adjustable inductor $L_{a}$. In series with several of the brushes are resistors, some shunted with capacitors, as shown.

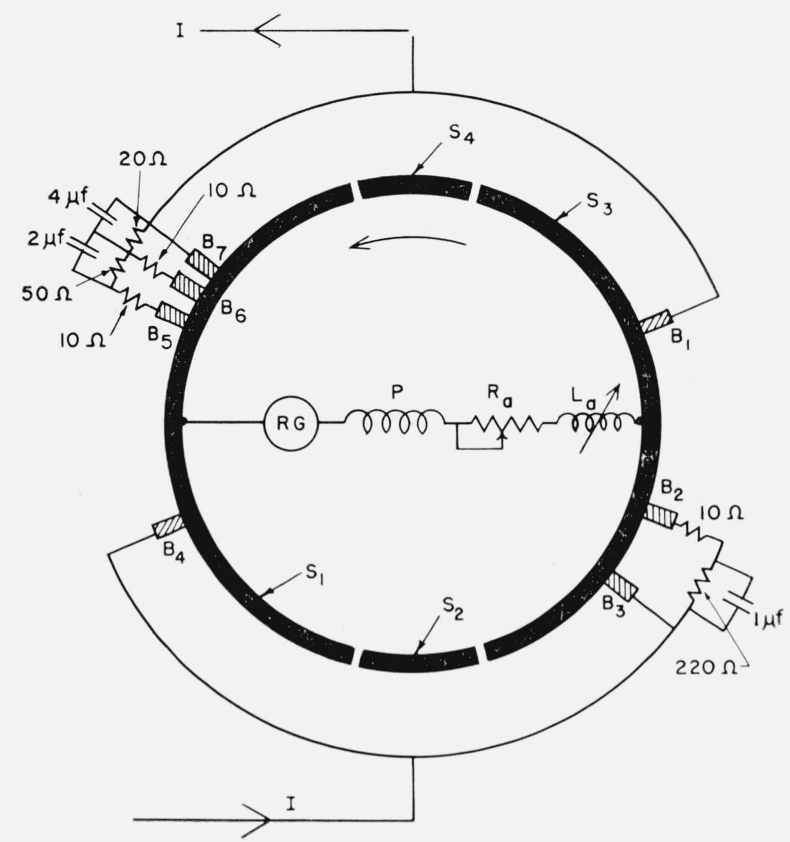

FIgURE 37. Details of connections to primary reversing switch.

In the position shown in figure 37 the circuit within the reversing commutator network is shorted. The current, $I$, divides and flows through brushes $B_{3}$ and $B_{4}$ into the long copper segments and out through brushes $B_{1}$ and $B_{7}$. The conductors that connect brushes $B_{1}$ to $B_{7}$ and brushes $B_{3}$ to $B_{4}$ are of low resistance and provide parallel shorts on the circuit containing the primary of the mutual inductor.

As the segments rotate with respect to the brushes, in the direction shown by the arrow, a position will be reached where brushes $B_{3}$ and $B_{7}$ pass from the long segments, $S_{1}$ and $S_{3}$, to the short segments, $S_{2}$ and $S_{4}$, while the remaining brushes remain in contact with the long segments. In this position the long segments are no longer connected directly together, but there are three parallel paths through resistors that connect them. These paths are through $B_{4}$ to $B_{2}$ and through $B_{5}$ and $B_{6}$ to $B_{1}$. As the commutator continues to rotate the parallel paths open up at $B_{6}, B_{5}$ and finally at $B_{2}$, after which the two parts of the primary circuit are in series. 
The purpose of the extra brushes $B_{2}, B_{5}$, and $B_{6}$ is to limit any voltage rise that will appear across the reversing switch in case the currents in the two parts of the primary circuit are not exactly equal when the short-circuit is removed. The first step in the removal of the short-circuit leaves parallel resistance paths of 30,80 , and 230 ohms, and these are opened in that order. To make the changes in resistance less sudden, capacitors were shunted across part of the resistance in each path; this, in effect, gives the equivalent of a low initial but quickly increasing resistance to each branch. Moreover, the motion of the brushes off the copper segments gradually decreases the areas of contact, which results in gradual increases in brush resistance rather than sudden openings.

No effort is made to apply the short-circuit in steps. However, both when the short is applied and removed, the peak value of the potential difference across the commutator always remains less than the maximum that can be satisfactorily compensated by the vacuum-tube chokes.

\section{(c) The Balancing Resistor}

For all the electrical measurements the circuit is balanced by varying a shunt on the standard resistor. This resistor is of the double-walled type [17] used at this Bureau for maintenance of the ohm, mounted in a well-stirred oil bath whose temperature is thermostatically controlled. In this bath, with the measuring current flowing through it, the temperature of the resistor is about $0.5 \operatorname{deg} \mathrm{C}$ above that of the bath. Hence it is necessary to standarize the resistor in its own oil bath and with an equal test current. For the inductor and frequency that are used, a shunt of about $105 \mathrm{ohms}$ is required. For this shunt the adjustable arm of a "direct-reading ratio set" [18] is used in series with a plug box. This ratio set is so constructed that its resistance can be accurately changed in steps of $0.001 \mathrm{ohm}$ from 99.5 to $100.5 \mathrm{ohms}$, and the remaining resistance of the shunt is obtained from a plug box with 0.1-ohm steps. A change in resistance of the shunt of 0.01 ohm corresponds approximately to a change of 1 part in a million in the parallel resistance. No temperature control is required for the shunt unless the temperature varies several degrees from that at which the shunt resistance is determined. This determination is made by opening the shunt and measuring the resistance across the break. (d) Current Reversing Switch

A switch in the primary circuit enables the operator to reverse connections to all batteries. All of these reversals are obtained with a motordriven multiple rotary switch, which interchanges connections at $m$ and $n, p$ and $q$, and to the magnetizing coils of inductor generator $J$, figure 32 . This switch is so arranged that reversals will occur in the proper sequence and that the amplifiers and galvanometer are properly protected during reversal. To obtain the reversal the operator has only to operate a push button, the switch stopping automatically at the end of the reversal.

The purpose of the reversals of the batteries is to eliminate the effect of possible thermal or other extraneous emf's in the measuring circuit that are not reversed with the measuring current. In balancing the circuit, the galvanometer is not brought to its open-circuit position but the resistance is adjusted until the galvanometer reading is the same for both directions of the main primary current. The difference of the reading of the galvanometer from its open-circuit reading shows that the stray emf's amount ordinarily to 2 or $3 \mu \mathrm{v}$ at most. These stray emf's are usually very constant in magnitude, and their effect is eliminated in so far as they remain constant between successive reversals of the batteries.

\section{Electrical Measurements}

\section{Location of the Inductor}

Construction of the mutual inductor and of all components of the measuring circuit was finished by the fall of 1938 . Electrical measurements with the completed apparatus were begun in October of that year. While making these electrical measurements it was desirable to have the inductor in such a location that its value would not be appreciably affected by magnetic materials in its neighborhood. For the greater portion of the first 2 -yr period the inductor was mounted on a special outdoor pier designed to support it above ground and at some distance from any building. This pier was entirely of wood, the parts being fastened together by wooden pegs and glue. The only metal parts were brass straps and bolts used to anchor the pier to the concrete footings and to the side of the building. 


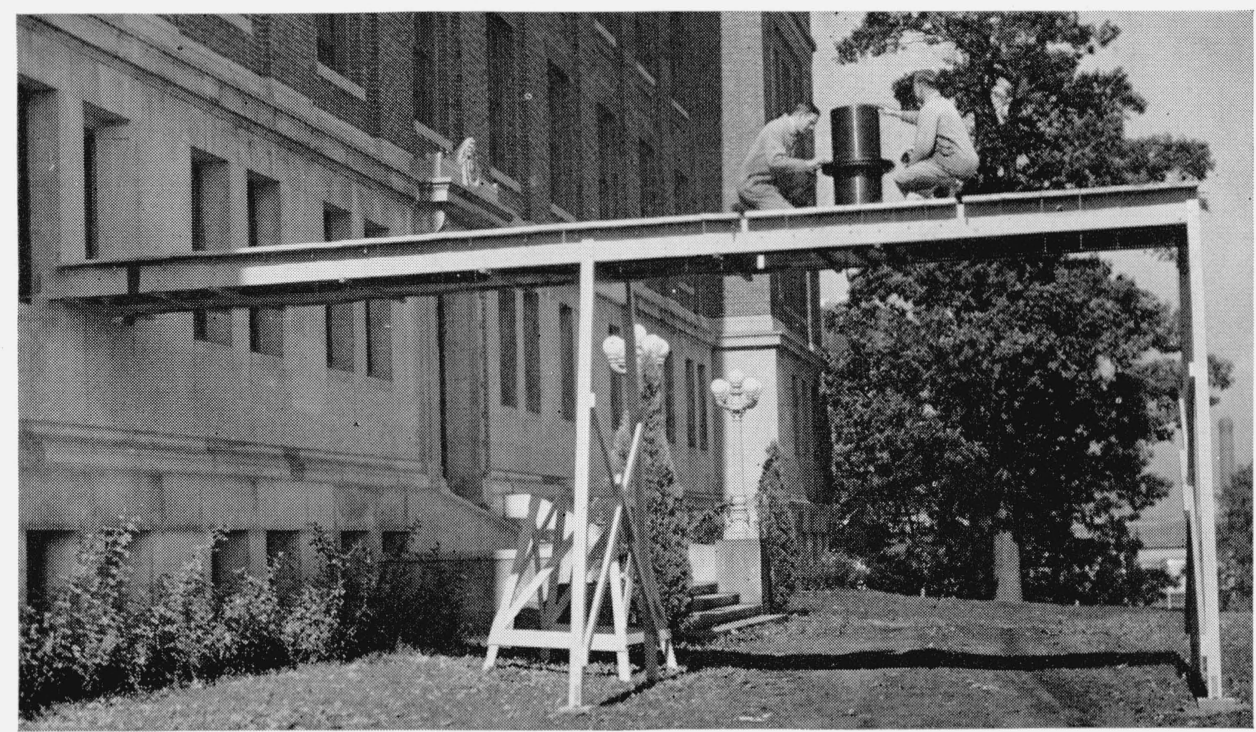

FIGURE 38. Outdoor pier, with wooden model showing location of mutual inductor.

The pier is shown in figure 38, a full-scale wooden model showing the location of the inductor, which was about $8 \mathrm{~m}$ from the building and $4 \mathrm{~m}$ above the ground. The nearer cast-iron lamp standard at the side of the steps was nearly $10 \mathrm{~m}$ from the inductor. A double-walled wooden box, filled with cotton batting, was mounted around the inductor, and a canvas tent over the box gave protection against the weather. Strapped to the underside of the pier were wooden ducts that were used to circulate dry air from the building through the inductor housing. Electrical connections to the inductor were by means of twisted pairs of rubber-covered copper wire attached to the underside of the pier.

An effort was made to control the temperature of the inductor at $23^{\circ} \mathrm{C}$ by means of the dry air circulated from the building. This was not very satisfactory, and it was found necessary to measure the temperature coefficient of the inductor and to correct for departures of the temperature from $23^{\circ} \mathrm{C}$. The determination of the temperature coefficient was made electrically. A series of measurements extending over 4 days was made with no change except for the temperature of the inductor and the corresponding value of resistance required for an electrical balance. The data obtained are shown in figure 39, where the changes in inductance in parts per million are plotted as ordinates against the temperature of the inductor. The slope of the curve determined from these data is $5.7 \mathrm{ppm} /{ }^{\circ} \mathrm{C}$. This is somewhat larger than had

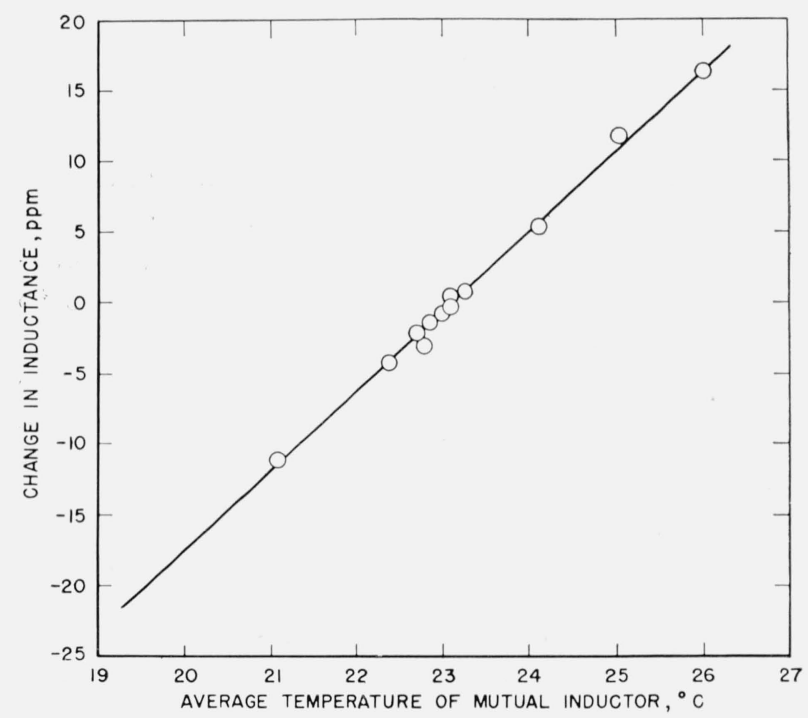

Figure 39. Date for obtaining temperature coefficient of inductance.

been expected, but probably changes in temperature of the wire on the primary change the stress it applies to the porcelain, and this produces changes in diameter in addition to those resulting from temperature changes of the porcelain.

In September 1940 the inductor was moved from the outside wooden pier and mounted on a wooden platform in one of the rooms of the Electrical Building, in a wing that had been constructed to be fairly nonmagnetic, and all magnetic material was carefully removed from the vicinity of the mounted inductor. To accomplish this 
result, the window frames were taken apart and then reassembled with brass screws, the floor was removed and replaced with a cement floor made with glass sand and nonmagnetic cement, and the walls were replastered with nonmagnetic plaster. The amount of resistance required to balance the circuit at $23^{\circ} \mathrm{C}$ did not change when the inductor was brought to its new location, and consequently it is believed that the value of inductance is not appreciably affected by any magnetic material in the building.

\section{Alinement of the Inductor}

The indoor platform to which the inductor was moved in 1940 has been employed without change since that date. Whenever the inductor is remounted on this platform, the primary lead wire and the secondary must be realined. The first step in this process is to set the primary vertical by means of a spirit level placed on the top surface of the porcelain form. The vertical primary lead wire and connectors are then secured in approximately the correct position along the front of the cylinder. The secondary is next suspended in place and its height adjusted so that the center of its channel coincides with the plane $x=0$, the location of the latter being known with reference to turns on the primary. A dial indicating gage equipped with a conical pin is used to measure the axial distance between the surfaces of the secondary form and the space between certain wires on the primary. Variations in glass thickness are allowed for. The last step in the alinement procedure consists in setting the primary lead wire vertical. For this purpose a plumb line is hung in front of the cylinder. A telescope mounted across the room is sighted on the plumb line, and the wire is moved until its image alines with that of the plumb line. The inductor is then enclosed within a double box, the inner one of which has a wooden fan at the top as shown in figure 40, this being a photograph of the inductor with the fronts of the enclosing boxes removed. The air-stirring fan is driven by a long cord extending to a motor located $6 \mathrm{~m}$ away from the inductor. The inductor rests upon an annular wooden ring supported about $10 \mathrm{~cm}$ above the bottom of the inner box. This permits the fan to draw air up through the center of the primary and discharge it downward along the outside. Thermocouples of negligible magnetic effect are attached to the porcelain form and enable its temperature to be measured from a convenient external location.

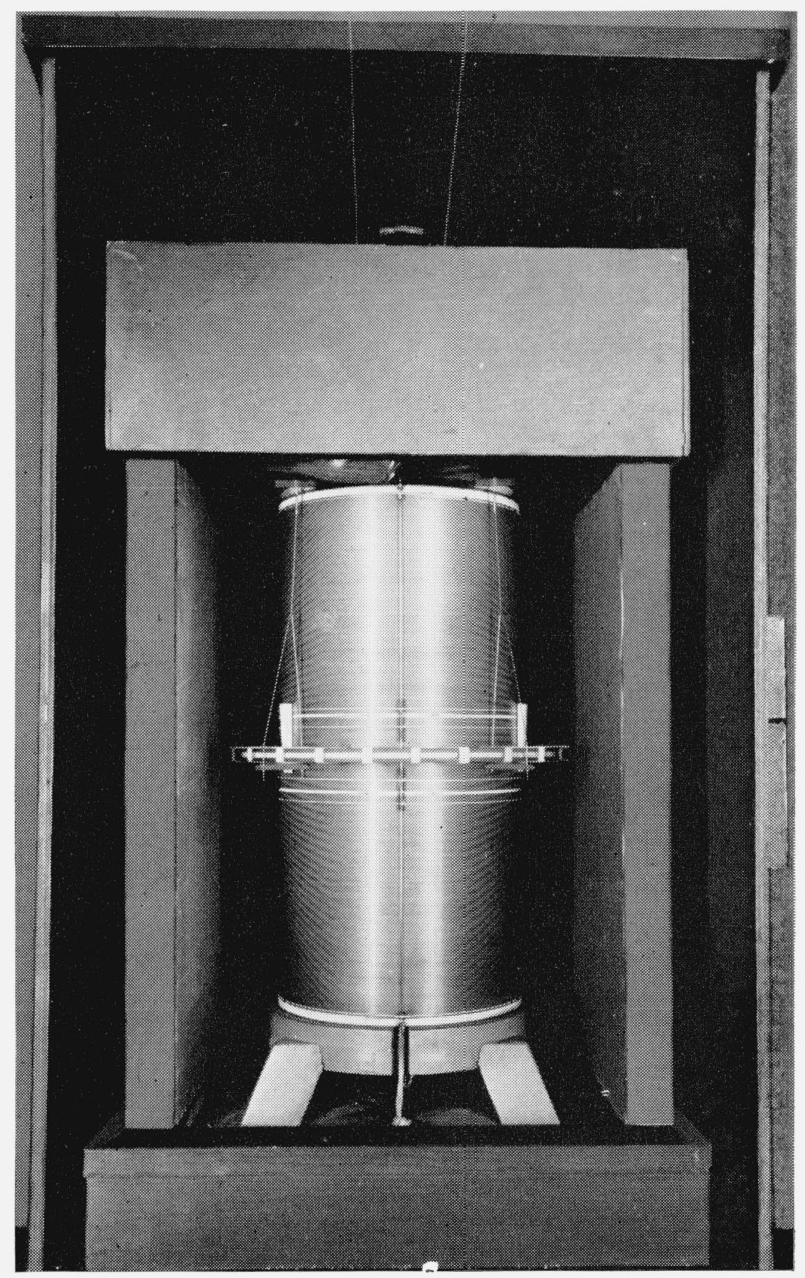

Figure 40. Inductor with front of double box removed.

\section{Adjustment of the Primary Circuit}

Before continuing with a description of the procedure followed in making an absolute resistance measurement, it is necessary to reexamine conditions that exist in the primary circuit.

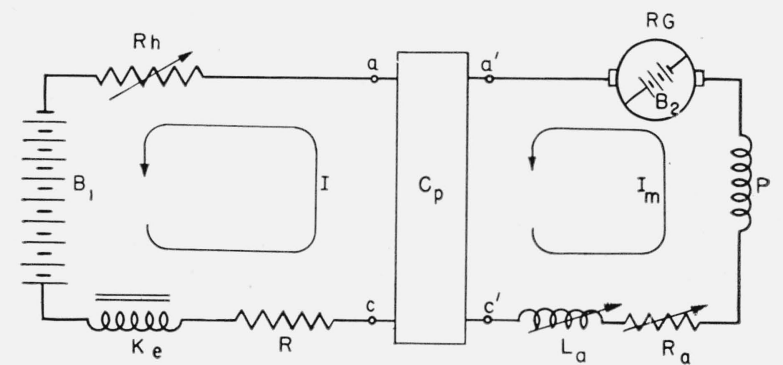

Figure 41. Schematic diagram showing the dual current paths in the primary circuit. 
Figure 41 is a simplified diagram drawn to illustrate the points presently involved. From what has been said before it is evident that for two-thirds of the time $I$ and $I_{m}$ are independent, and their values are determined by their respective batteries, $B_{1}$ and $B_{2}$, and associated equipment. During a period when the points $a$ to $c$ are shortcircuited, the reversing generator reverses the direction of $I_{m}$ through the primary of the mutual inductor in accordance with the middle curve of figure 33, while the shaft is moving say from $30^{\circ}$ to $150^{\circ}$. During the same time the rotary switch, $C_{p}$, reverses the connections at $a, a^{\prime}$, $c$, and $c^{\prime}$. In the meanwhile, $I$ is held constant by various current stabilizing devices. The effect of the vacuum-tube chokes is to diminish by a factor of 400 or more sudden emf changes of $0.1 \mathrm{v}$ or less appearing across $C_{p}$, and this is initially equivalent to an increase in the effectiveness of the iron-cored choke coil by the same factor. In figure $41, K_{e}$ therefore represents an equivalent choke of $400 \times 7$, or $2,800 \mathrm{~h}$. It may be regarded as a large energy reservoir. When the shaft advances from $150^{\circ}$ to $160^{\circ}$ (fig. 33), the rotary switch quickly removes the short circuit in three distinct steps, at the end of which time the primary circuit is series connected and remains so while the shaft moves from about $160^{\circ}$ to $210^{\circ}$. If current $I_{m}$ is not exactly equal to $I$ when the short circuit is removed, it is nevertheless quickly forced to assume equality with it, the requisite energy being supplied by $K_{e}$. The energy transfer does not take place, of course, without some slight influence upon the value of $I$, and the effect will be examined shortly. Approximately in the middle of the series interval, when the shaft angle is about $180^{\circ}$, the secondary connections are reversed at the time marked $X$ in figure 33. At this moment the instantaneous value of $I$ in the primary circuit is $i_{x}$. Current $i_{x}$ enters into the ratio $i_{x} / I_{a}$ appearing in eq 2 , and as was pointed out in section II, this current ratio is difficult to measure directly with the required accuracy. Electronic amplifying and measuring devices must be employed, and the emf's to be measured are of the same order of magnitude as the inherent noise in tube circuits. Such electronic measuring devices do, however find application in the present problem. A cathode-ray oscillograph equipped with amplifiers is connected to the points $a$ and $c$ of figure 41 . A moderate vertical sensitivity of $0.1 \mathrm{v} / \mathrm{in}$. is employed, and consequently voltage disturbances of $5 \mathrm{mv}$ or more are readily discerned. If the setting of rheostat $R h$ is slowly changed while the apparatus is running, the ratio of $I$ to $I_{m}$ at the moment of short circuit removal may be judged from the oscillograph response. If the currents are matched, there is no discontinuity in the oscillograph trace when the short is removed. If, for example, $I$ is set 15 ma above the matched value in the vicinity of 0.8 amp, a "table" $1 \mathrm{in}$. high $(100 \mathrm{mv})$ will appear in the oscillograph trace of emf during the period of primary series connection. During the $15-\mathrm{ms}$ short-circuit periods, random emf variations appearing across the points $a$ to $c$ are very small, never exceeding 10 $\mathrm{mv}$. A $10-\mathrm{mv}$ rectangular voltage pulse of $15-\mathrm{ms}$ duration will change $0.8 \mathrm{amp}$ in a $2,800-\mathrm{h}$ circuit by less than 1 part in $10^{7}$. If the voltage disturbance appearing across the points $a$ to $c$ during the series-connected period is likewise small, it is apparent that the instantaneous value of primary current will never be displaced from its value averaged over the complete cycle by as much as 1 part in $10^{7}$. No difficulty is experienced in setting $R h$ so that the height of the voltage "table" appearing during the 7 -ms series period is within $10 \mathrm{mv}$ of the zero level of emf established during the short-circuit period. The problem centers chiefly around events that occur during the 1.5-ms transition period when the connections are changing from short-circuit to series. As stated earlier, the switch $C_{p}$ removes the short in three steps, and at each of these steps an oscillating emf disturbance is set up that may considerably exceed $0.1 \mathrm{v}$ in amplitude if the resistance and inductance of the reversing generator circuit differ from the values for which the generator was designed. By adjusting $R_{a}$ and $L_{a}$, particularly the latter, the amplitude of these oscilations may be reduced to less than $0.1 \mathrm{v}$, and their time integral value may be visually adjusted so nearly equal to zero that no appreciable net emf disturbance remains. When the adjustments of $R h, R_{a}$, and $L_{a}$ are so made that the net emf disturbance displayed on the oscillograph screen is substantially zero, it is inferred that the current ratio $i_{x} / I_{a}$ differs from unity by a negligible amount. The effectiveness of the current-stabilizing devices is illustrated by the following test. The circuit is first balanced with $I$ and $I_{m}$ matched as closely as possible. Current $I$ is then raised 
about $15 \mathrm{ma}$ with the result that the voltage "table" appearing during the series period is about $100 \mathrm{mv}$ high. This emf disturbance, lasting for about $7 \mathrm{~ms}$, changes the main balance by about $3 \mathrm{ppm}$. If current $I$ is lowered $15 \mathrm{ma}$ below the matched value, the voltage table is $100 \mathrm{mv}$ negative, and the main balance is off by about $3 \mathrm{ppm}$ in a direction opposite to the first case. If this same test is repeated with the vacuum-tube chokes disconnected, the change in balance amounts to about $18 \mathrm{ppm}$. In normal operation the voltage impulse is much less than $700 \mathrm{mv}$-sec, and consequently the current ratio $i_{x} / I_{a}$ differs from unity by very much less than $3 \mathrm{ppm}$.

\section{General Procedure for Making an Absolute Measurement of Resistance}

No absolute measurements are attempted if the inductor's temperature is nonuniform or if its mean temperature has been changing rapidly during the preceding $24 \mathrm{hr}$. If the equipment has been idle for some time, temperature differences amounting to several tenths of $1 \mathrm{deg} \mathrm{C}$ may exist between the top and bottom of the porcelain form. After the air-stirring fan is operated for $6 \mathrm{hr}$ or more, these temperature inequalities are reduced to $0.01^{\circ} \mathrm{C}$. When temperature conditions are satisfactory, the main driving motor is started and the equipment is allowed to warm up for 10 or 15 min with the normal test current flowing in the measuring circuit. When thermal equilibrium has been reached, the automatic speed control is put into operation and the driving-power level adjusted so that the mechanical system hunts symmetrically about the synchronous position. With the cathode-ray oscillograph used in place of the galvanometer, the excitation and phase angle of the inductor generator are adjusted to produce a minimum amplitude of alternating voltage in the galvanometer circuit. The oscillograph is then connected across the primary reversing switch, and the rheostat, $R h$ (fig. 32), is adjusted to produce a current match in the two portions of the primary circuit. If necessary, the auxiliary inductor, $L_{a}$, is adjusted to reduce to a minimum the voltage disturbance during the transition period from short-circuit to series connection in the primary. The circuit is then balanced by varying the shunt on $R$ to bring the galvanometer to a selected false zero in the vicinity of its opencircuit zero. Next, all battery connections are reversed using the remote-control switch described in section VII, 3, (d). After quickly checking the speed-control adjustment and the primary current match, the shunt, $S h$, is again adjusted to bring the galvanometer to the same false zero that was used in the previous balance. There is some random movement of the galvanometer spot because of momentary fluctuations in speed; these movements, amounting to a few millimeters, are integrated by eye in the process of making a balance. The time required to perform this integration will be referred to later as the galvanometer observing time. The mean of the two values of $S h$ is recorded, and this is the setting of the shunt that would have been required had there been no thermal or other irreversible emf's in the galvanometer circuit, and had the galvanometer been brought to its mechanical zero. Although it is not necessary that the two values of $S h$ be equal, experience with the small thermal emf's ordinarily present quickly indicates how the false zero may be chosen so as to make the two values of Sh practically equal. These balances are repeated several times and a mean shunt value, $S$, arrived at. The mean temperature of the inductor is then measured. From the known temperature coefficient of the mutual inductor it is possible to calculate the value, $R_{e}$, of the equivalent unshunted resistance that would have balanced the mutual inductance had the inductor been at the standard temperature of $23^{\circ} \mathrm{C}$. The value of this equivalent resistance is given by the following expression:

$$
R_{e}=R\left(\frac{S}{S+R+r_{1}+r_{2}}\right)[1-\alpha(t-23)] .
$$

Here $R$ is the four-terminal value of the standard at the temperature and load conditions as used in this experiment, $r_{1}$ and $r_{2}$ are the values of its potential lead resistances, $S$ is the mean value of the shunt required to balance the inductor when the latter is at $t^{\circ} \mathrm{C}$, and $\alpha$ is $+5.7 \times 10^{-6}$.

\section{Results Obtained}

Under favorable conditions two observers can balance the measuring circuit and obtain the same balance to about half a part in a million, and even under less favorable conditions they usually agree to 1 part per million. However, a series of readings taken at intervals of 1 or more days shows an 


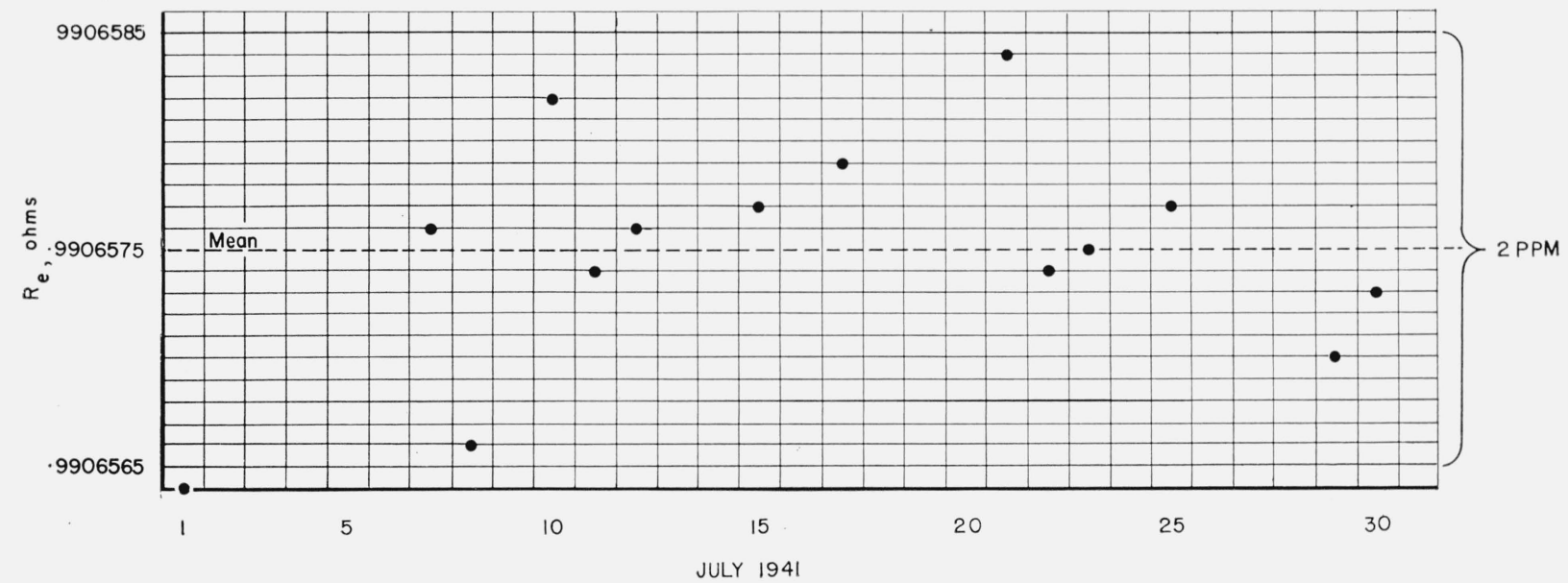

FIGURE 42. Values of resistance required to balance the circuit during a 1-month period.

apparent erratic scattering of balances, which scattering, however, seldom exceeds \pm 2 ppm from the average of a considerable number. As an example, figure 42 shows all readings taken in the month of July 1941. The ordinates represent the values of the resistance, $R_{e}$, required to balance the circuit on the days shown. Throughout this paper all resistance values, unless otherwise stated, are in terms of the absolute unit adopted by international agreement on January 1, 1948, regardless of what unit may have been used when the original data were taken. The scattering evident in figure 42 resulted from uncertainties in determining the electrical balance, possible errors in correcting for the effect of temperature on the value of inductance, and possible changes in resistance, inductance, and frequency during the month. Over a period of a month any change in resistance was almost surely negligible, and it is also believed that the frequency was constant to 1 part in $10^{7}$, as it was determined by crystal oscillators. The average value of the shunted resistance for the month was 0.9906575 with variations from this mean of not over about $\pm 1 \mathrm{ppm}$.

An extended series of determinations of the values of resistance required to balance the measuring circuit was started in October 1938, and this series has been continued up to the present time except for the war period. The results of these measurements are shown in figure 43. The plotted points represent the monthly averages in cases where at least five determinations were made during the month. Where fewer than five readings were taken during a month, the point plotted is for a group of at least five readings, plotted at the center of the time interval covered by the readings.

Figure 43 does not show the results of all measurements made during the period covered. On several occasions the resistance required for balance would start decreasing at a rather constant rate of 3 or $4 \mathrm{ppm}$ per month, and this change would continue for several months if it were allowed to do so. Although no explanation of the phenomenon was obtained, it was found that the drift was associated with a biasing battery in the amplifier that furnished the $1,000-\mathrm{c} / \mathrm{s}$ frequency for the control of the constantspeed shaft. This $C$-battery was replaced, the bias being obtained instead as a potential drop across a resistor, and the difficulty was eliminated. The 1,000-c/s emf was obtained from a circuit that was used to drive a synchronous clock, but the readings of the clock were apparently unaffected. The difficulty, however, threw a doubt upon the frequency, and it was necessary to obtain an independent determination during proper functioning.

As a test of the $1,000-\mathrm{c} / \mathrm{s}$ frequency, the rate of the constant-speed shaft was checked by comparison with the Shortt Synchronome clock of the Time Section of this Bureau. The clock so operates that every $30 \mathrm{sec}$ an electrical impulse is sent from the free pendulum to a relay that controls the slave clock. Without using the relay contact, the impulse to the relay coil was impressed on the grid circuit of a gas-discharge tube in such a way as to fire the tube. A spark-coil in the plate 


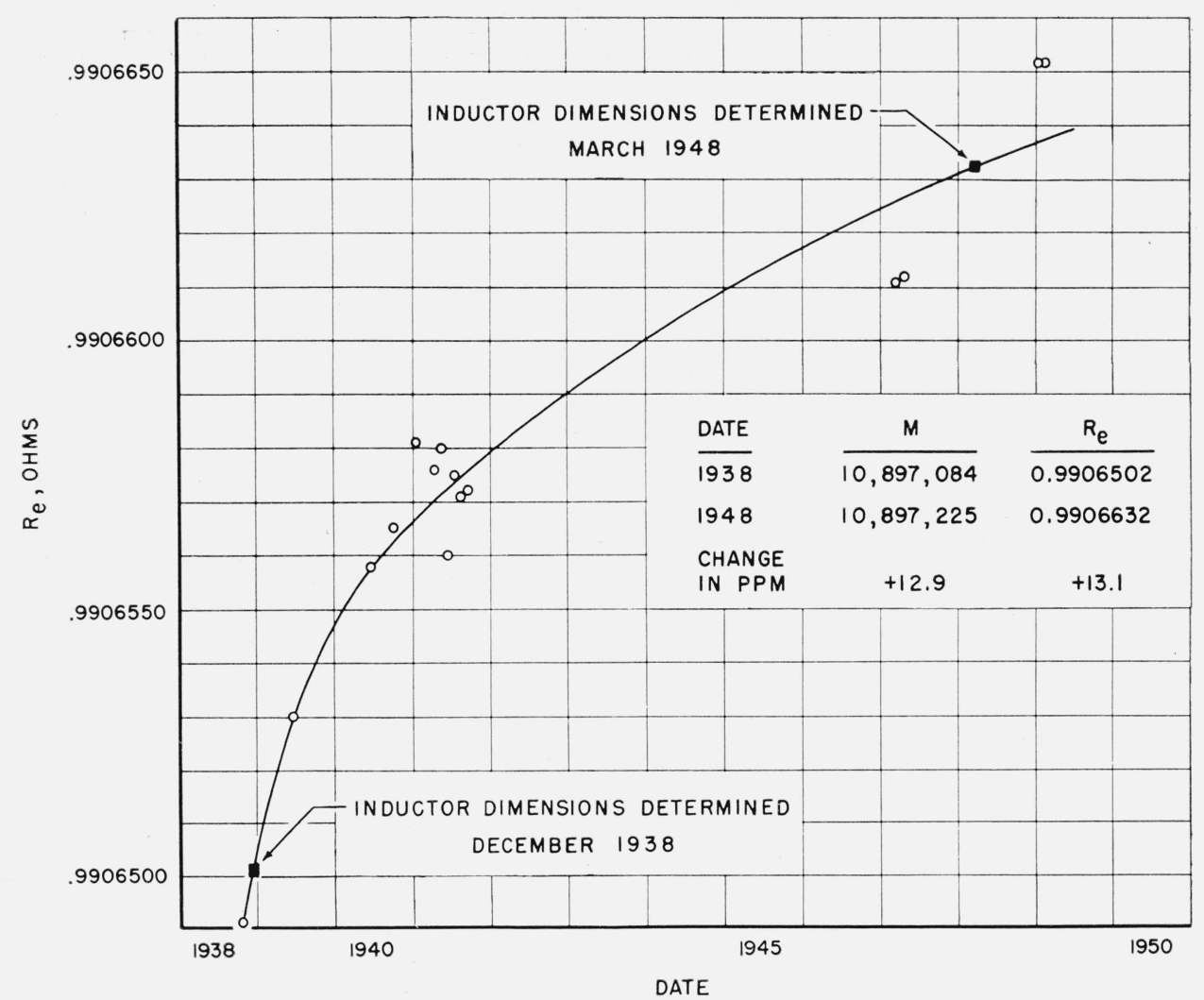

Figure 43. Mean values of resistance required to balance the circuit during the period October 1938 to February 1949.

circuit of the discharge tube produced a high voltage that was made to cause a spark from a stationary insulated plate to a rotating point on the constant-speed shaft. With exact synchronization this spark would occur at the same point of the insulated plate every eleventh spark, i. e., every $5 \frac{1}{2}$ min. With the sparking circuit open except for every eleventh impulse and a piece of electrosensitive paper on the stationary plate to record the positions of the sparks, the rate of travel of the spark gave an accurate determination of the rate of the shaft as compared with that of the clock. The latter was determined by checking against signals from the Naval Observatory.

Several tests of the speed of the shaft were made in this way. By taking observations over an interval of about $100 \mathrm{~min}$, the rate could be determined to about 2 parts in $10^{7}$, which was as accurate as the known rate of the clock. To this accuracy the rate of the $1,000-\mathrm{c} / \mathrm{s}$ emf was found to be correct. In the formula for resistance in terms of mutual inductance and time the value of $n$ is therefore used as

$$
n=1,000 / 44 \text { rps. }
$$

An examination of figure 43 shows that either the inductance has increased from the first measurements by about $13 \mathrm{ppm}$, or that the unit of resistance has decreased by an equal amount; or perhaps that there have been changes in both. Since the relative change was most rapid soon after completion of the inductor, the form of the curve suggests that the change was primarily in the inductance. The resistors that were used to maintain the unit of resistance during this time interval were constructed in 1933 and could hardly have been changing in such a way as to explain the curve.

If the change in the ratio of inductance to resistance were the result of an increase in mutual inductance, the primary of the inductor must have increased in dimensions. Such a change is entirely possible, as the copper wire might have yielded slightly and allowed an increase in diameter, or the porcelain cylinder might have increased in size because of the familiar "humidity expansion" of material of this kind. A relieving of tension in the wire would expand the diameter and shorten the porcelain cylinder, whereas a humidity 
expansion would increase both diameter and length. The latter change was found to have actually taken place.

The values of diameter and pitch given in the section on measurement of the inductor were determined in 1947 and 1948. A similar, although not as thorough, set of measurements had been made in December, 1938, and was used in calculating the results given at that time to the International Committee on Weights and Measures. A comparison of the two sets of dimensional measurements shows that in the decade the primary of the mutual inductor increased in average diameter by $12 \mathrm{ppm}$, increased in average pitch by $11 \mathrm{ppm}$, and that no significant change took place in the dimensions of the secondary. Since for small changes the inductance was found to change as the 1.92 power of the primary diameter and inversely as the 0.92 power of the primary length, the above-mentioned changes in dimensions show that the inductance has increased by $12.9 \mathrm{ppm}$. An examination of figure 43 shows that in the same interval of time the inductance as measured in terms of the unit of resistance increased by $13.1 \mathrm{ppm}$. This means that the apparent change in inductance in terms of resistance can be accounted for almost entirely by the increase in dimension of the primary helix, and that there has been no significant drift in the unit of resistance as maintained by means of wirewound resistors. The excellent agreement between the change in the mutual inductance as measured mechanically and as measured in terms of the unit of resistance is undoubtedly somewhat fortuitous, as either of the mechanical measurements could have been in error by 1 or $2 \mathrm{ppm}$.

By using the value previously given for the mutual inductance in 1948 and using $n$ equal to $1,000 / 44$, we obtain

$$
\begin{aligned}
R=4 n M & =4 \times \frac{1000}{44} \times 10,897,225 \\
& =990,656,800 \mathrm{cgsm} \text { units of resistance } \\
& =\mathbf{0 . 9 9 0 6 5 6 8} \text { absolute ohm }
\end{aligned}
$$

This is the value of the resistor used in balancing the electrical circuit for the measurement of resistance in terms of inductance and time. However, the value of this resistor in terms of the absolute ohm maintained with wire-wound standards was found to be $\mathbf{0 . 9 9 0 6 6 3 2}$, which is larger than the measured value given above by 6 microhms. The values assigned to the wirewound standards were those assigned on January 1, 1948, in terms of the unit selected by the International Committee on Weights and Measures as being the most probable on the basis of measurement prior to 1939. In terms of the measurements herein reported, the unit that they selected appears to have been too small by 6 microhms.

The unit of electrical resistance has been maintained at this Bureau by means of a group of wirewound resistors [17], by assuming that the average value of ten resistors remains constant. Such resistors have been very constant with respect to one another, but there has been no real assurance that they are not all changing in resistance at the same rate. During the period 1938-48 the average total relative change of each of the ten resistors, without regard to sign, was $0.5 \mathrm{ppm}$, the maximum being $1.0 \mathrm{ppm}$. These small relative changes lead to the belief that the average of the group is constant to at least the same order of accuracy. The results given in the preceding paragraphs seem to bear out this belief, and they constitute the first satisfactory evidence as to the accuracy with which the unit of resistance has been maintained at this Bureau.

It is expected that this series of measurements of resistance in terms of inductance and time will be continued. When combined with occasional measurements of the dimensions of the inductor, this will in effect constitute a maintenance of the unit of resistance by means of the cgs units in terms of which it is defined.

\section{Summary of Numerical Results}

A preliminary report of numerical results obtained by the method described in this paper was submitted to the International Committee on Weights and Measures under the date of December 28, 1938, and appears in a publication [19] of that organization. The results submitted were based upon electrical and dimensional measurements made in October to December of that year. No correction for variations in pitch of the mutual inductor was made at that time, but it was reasonably certain that the pitch errors were small. The preliminary results given at that time stated that

1 NBS Washington unit $=1.000485$ absolute ohms. 
Regarding the postwar electrical and dimensional measurements as a new determination of the ratio of the units, the above relation is now written:

1 NBS Washington unit $=1.000482$ absolute ohms.

Figure 44 is presented in order to clarify the relationships that exist between various resistance units. Although this figure is not drawn to scale, it is intended that it indicate the differences in size of the several units, a small unit being shown

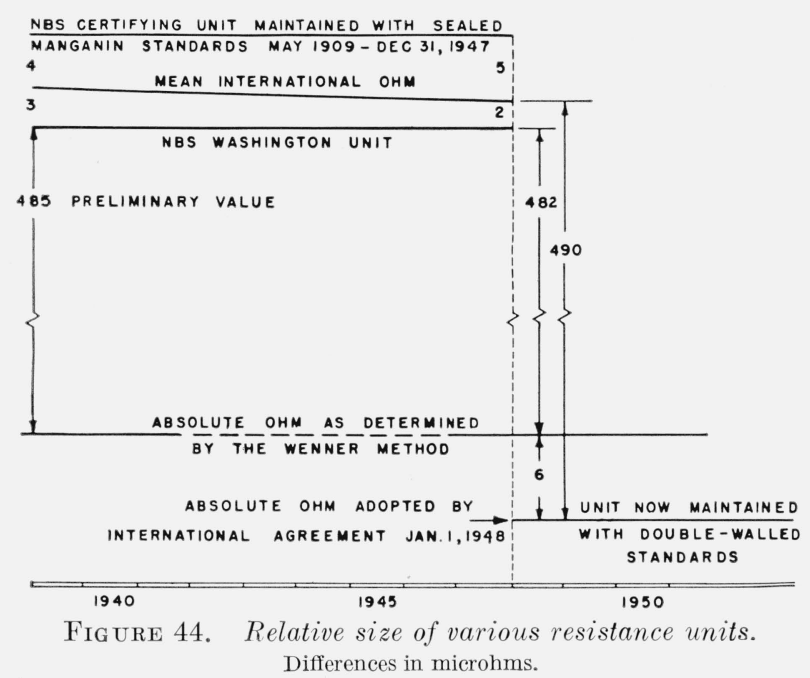

at the bottom of the figure. In the interval 193747 the difference between the Washington Unit and the Mean International Ohm decreased by 1 microhm, as indicated in the figure. The results of postwar absolute resistance measurements may be expressed in the following optional forms:

\section{1 mean international unit $(1947)=1.000484 \mathrm{ab}$ - solute ohms,}

or

\section{$1 \mathrm{ohm}$ as presently maintained at $\mathrm{NBS}=0.999994$ absolute ohm.}

The results of these measurements may also be expressed in terms of numerical values of resistance assigned to standard resistors numbers 78,82 , and 84. These resistors were submitted to the International Bureau in 1938 for comparisons of the national units and are of the large doublewalled sealed type, originally constructed at this Bureau in 1933. These three resistors, and seven others of similar construction, now constitute the group of 10 standards that maintain the unit of resistance in the United States. Data for the three resistors are assembled in table 12 .

TABLE 12. Resistance of standard resistors 78,82 , and 84

\begin{tabular}{|c|c|c|c|c|}
\hline Item & Description & No. 78 & No. 82 & No. 84 \\
\hline 1 & $\begin{array}{l}\text { Values in Washington units at } 20^{\circ} \mathrm{C} \text {, } \\
\text { as given in NBS certificates } \\
\text { dated November } 22,1938 \text {, sent to } \\
\text { International Bureau of Weights } \\
\text { and Measures......................... }\end{array}$ & 0.999526 & 0.999556 & 0.999532 \\
\hline 2 & $\begin{array}{l}\text { Values in absolute ohms at } 20^{\circ} \mathrm{C} \text {, } \\
\text { as given in Preliminary Report } \\
\text { dated December } 28,1938\end{array}$ & 1. 000011 & 1. 000041 & 1. 000017 \\
\hline 3 & $\begin{array}{l}\text { March } 1948 \text { values at } 20^{\circ} \mathrm{C} \text {, in terms } \\
\text { of the unit now maintained }\end{array}$ & 1.000015 & 1. 000044 & 1. 000020 \\
\hline 4 & $\begin{array}{l}\text { March } 1948 \text { values, absolute ohms at } \\
20^{\circ} \mathrm{C}\end{array}$ & 1. 000009 & 1. 000038 & 1. 000014 \\
\hline
\end{tabular}

In the above table the numerical values given opposite items 1 and 3 have been taken from the records of the Resistance Measurements Section of this Bureau. Absolute resistance measurements made during this investigation employed 1-ohm standard resistor No. 82 during the early portion of the work, whereas resistors No. 67 and No. 76 were used during the postwar work. The data for items 2 and 4 were obtained by comparing the three resistors with the standards used in the absolute measurements. No conclusions regarding the stability of these resistors should be drawn from these data, because the decrease in value results primarily from revisions that have been made in the values of certain correction terms utilized in calculating the effective value of mutual inductance. These revisions, made subsequent to issuance of the preliminary report, will be discussed in the section that follows.

\section{Discussion of Numerical Results}

(a) Comparison with Preliminary Results

The ratio of the NBS Washington unit to the absolute unit as determined by these experiments was given the preliminary value of 1.000485 in 1938, and is herein assigned the final value of 1.000482. The difference between the two results is influenced by several factors, the most important of which is associated with the corrections used in arriving at the effective value of mutual inductance, the most bothersome correction being that connected with distortion of the primary wire. 
In December 1938 this correction was taken as +6 ppm, but subsequent review of the problem has revealed that the original analysis was incorrect and that the correction should have been taken as $+0.5 \mathrm{ppm}$ following the line of reasoning set forth in this paper. This change of $5.5 \mathrm{ppm}$ is partially compensated by adjustments that have been made in the values of three other corrections. Except for the additional data upon which the pitch variation correction is based, these adjustments result from a critical review of prewar analyses rather than from changes in basic data. The net effect of these revisions is such that, had the present pitch correction been available in 1938, and had all corrections then been calculated in the manner now believed correct, the effective value of $M$ would have been $2.2 \mathrm{ppm}$ smaller than the value adopted in December 1938, and the ratio of the units would have been reported 1 NBS unit= 1.000483 absolute ohms. The close agreement between this figure and the postwar result is somewhat fortuitous, inasmuch as uncertainties in dimensional measurements of the inductor could easily account for differences larger than those encountered.

(b) Comparison of Final Results with those of Other Investigators

In preparation for the change in units ultimately made on January 1, 1948, several national laboratories made painstaking absolute measurements of resistance, beginning about 1933 . Complete details describing three distinctly different methods as carried out in three laboratories have been published by Hartshorn and Astbury [20], Vigoureux [21], Curtis, Moon, and Sparks [7], and Jouaust, Picard, and Hérou [22]. The final results ob- tained by these workers, together with that obtained in this investigation, are listed in table 13.

In table 13 the numerical results listed in the last column give the value in absolute ohms of the mean international ohm. They are calculated from values published in terms of the international ohm as maintained in the designated laboratories at the time of measurement. The international ohms used in the three laboratories mentioned differed from one another, and from the mean international ohm, by only a few ppm. The magnitudes of these differences during the period 1933 to 1939 are given in a publication of the International Bureau of Weights and Measures [23].

In the early part of 1939 the International Committee on Weights and Measures had before it the first four final figures given in table 13, and in addition, preliminary results from the present investigation; from Yoneda [24] at the Electrotechnical Laboratory, Tokyo; and from Zickner [25] at the Physikalisch-Technische Reichsanstalt, Berlin. Action by the committee was delayed by the war, but at a meeting of its Advisory Committee held in 1946 the value 1.000490 was selected as the best value for the ratio of the mean international ohm to the absolute ohm based upon the experimental work referred to above. The final results obtained in the present investigation differ from the value selected for international use by only 6 ppm and thus support the committee's selection.

As an interesting example of a different method for the absolute measurement of resistance, reference is made to the work of Nettleton and Balls [26], who carried out a measurement using

TABLE 13. Results of absolute resistance measurements

\begin{tabular}{|c|c|c|c|c|}
\hline Date of measurements & Reference & Laboratory & Method & $\begin{array}{c}\text { Value of } \\
\text { mean inter- } \\
\text { national ohm }\end{array}$ \\
\hline $1936 \ldots \ldots . .$. & Hartshorn and Astbury. & NPL a & Campbell. & $\begin{array}{c}\text { Ohms } \\
\text { 1. } 000505\end{array}$ \\
\hline 1933 to $1936 \ldots$ & Vigoureux. & NPL & Lorenz. & 1. 000499 \\
\hline $1936,1938 \ldots$ & Curtis, Moon, and Sparks. & NBS b & $\begin{array}{l}\text { Self-inductance standard and Maxwell capaci- } \\
\text { tance bridges. }\end{array}$ & 1. 000472 \\
\hline $1937 \ldots \ldots$ & Jouaust, Picard, and Hérou. & LCE。 & Modified Campbell. & 1. 000519 \\
\hline 1938 to $1949 \ldots \ldots$ & Present paper. & NBS & Wenner. & 1. 000484 \\
\hline
\end{tabular}

a National Physical Laboratory.

b National Bureau of Standards.

- Laboratoire Central d'Electricité. 
apparatus developed by them and based upon a method suggested by Rosa in 1909. As a matter of historical interest it may be remarked that in those early days Rosa and Wenner were independently seeking to develop a d-c method for the absolute measurement of resistance that would be as simple and direct as the Lorenz method, yet avoid the limitation of the latter due to the low emf produced by the homopolar generator. The results reported herein represent the culmination of many years' work on the part of Wenner and his associates in developing his approach to the problem. The method used by Nettleton and Balls was entirely independent but in many ways similar to Wenner's. Their work was carried out primarily to demonstrate the method, but with a few refinements could probably have furnished valuable additional data on the ratio of the international and absolute ohms.

\section{Performance of the Measuring Circuit}

\section{General Remarks}

When this project was started, the plans called for a determination of resistance in absolute units to a few parts in a million. Hence, whenever possible, sources of error were to be reduced to 1 part in 10 million or less. Factors that could not be so reduced were to be measured to that accuracy if possible. In the preceding sections a number of potential sources of error have been discussed. Besides those discussed, there were many others of the types common to all exact electrical measurements. Such, for example, are problems of adequate insulation, temperature corrections to resistors, heating caused by current flowing in the resistors and inductors, etc. It is believed that a detailed discussion of the steps taken to meet these difficulties is not necessary. There are, however, several possible sources of error peculiar to the method and apparatus that have not been considered in detail; a discussion of these follows.

\section{Discussion of Certain Sources of Error}

(a) Magnetic Permeability

The magnetic properties of all materials used in constructing the mutual inductor were tested at least qualitatively. Because of the relatively large masses of copper and porcelain used in constructing the primary, great attention was de- voted to these materials. Qualitative tests made on samples of the copper wire showed that it was diamagnetic; it was therefore certain that its susceptibility did not exceed $-100 / 10^{9}$, which was considered negligible. Quantitative observations were made on samples of porcelain cut from a block prepared from the same batch of clays as was used in making the primary cylinder. Using a torsion balance with the specimen hung in a field of about 1,000 oersteds, the susceptibility of the porcelain was found to be $+32 / 10^{9}$, which is negligible. Because the specimen tested may not have been representative of the large mass of porcelain used, the following supplementary test was devised. An auxiliary mutual inductor was constructed by using a wooden form about $110 \mathrm{~cm}$ long with an outside diameter slightly less than the inside diameter of the porcelain form. Channels $2.5 \mathrm{~cm}$ wide and $2.5 \mathrm{~cm}$ deep at each end of the form were filled with bunched windings, thus forming two separate secondary coils. The central 1-m space between these coils was filled with a single-layer helix, which formed the primary winding. The inductance between this primary and each secondary was about $15 \mathrm{mh}$. This auxiliary inductor with its two secondaries connected in series opposition was used to balance a low-range adjustable resistor in the absolute-resistance measuring circuit. The test inductor was so mounted that it could be lowered into the main inductor and held at various levels while electrical balances were made. If the permeability of the porcelain had differed from air by an appreciable amount, a measurable change in differential inductance would have occurred in the test inductor. No significant change in electrical balance was observed during the course of a series of observations taken as the bottom secondary coil of the auxiliary inductor was lowered from a coaxial position well above the main inductor to a position near the bottom. This test did not give data from which the susceptibility of the porcelain could be calculated, yet it is believed that if the susceptibility had been large enough to produce an error of as much as $1 \mathrm{ppm}$ in the value of $M$, it would also have affected noticeably the balance in the test just described.

(b) Alinement of Primary Lead Wire

When primary connections are made in the manner described in section VIII, 2 , it is obvious 
that the winding will contain an exact whole number of turns. The effect of sliding the top contact, for example, along the wire of the helix is not to change the number of turns, but to replace or counterbalance a tangential increment of the helix with a tangential component of the lead wire. If, for example, the top contact is moved by $1 \mathrm{~mm}$ in the direction to increase the length of wire in the helix, the length of the primary wire is increased by about $2 \mathrm{ppm}$ (the primary wire is about 500,000 $\mathrm{mm}$ in length), but there is actually a decrease in mutual inductance of between 2 and $3 \mathrm{ppm}$. An approximately equal change in inductance will also result from a sidewise motion of about $1 \mathrm{~mm}$ of one of the connecting jumpers. These experimentally determined results of movement of the contacts indicate that the method of alining the primary leads is adequate.

It might appear that the contact iaces, $K$ and $K^{\prime}$ of figure 6 , are so large that the inductance would depend upon the points of contact between $K$ and $K^{\prime}$ and the wires of the helix. Actually, however, any variation is negligible. If the jumper were to rock in such a way as to shiit one contact, say from the center to the edge of $K^{\prime}$, the effect of the change of about $1 \mathrm{~mm}$ in the length of the helix would be almost completely neutralized by an opposite lateral component of the current as it flows in $K^{\prime}$ to the point of contact.

\section{(c) Alinement of Secondary}

The alinement of the secondary coil with reference to the primary is accomplished mechanically as described in section VIII, 2. This can be done to a few hundredths of a millimeter, which is found to be adequate.

Aiter the secondary has been properly set, the adjustments are tested electrically. It is found that radially the secondary can be moved a millimeter without a measurable change in inductance. In the axial direction, a change of $1.5 \mathrm{ppm}$ results from a movement of $1 \mathrm{~mm}$ in either direction, and the adjustment within a few hundredths of a millimeter is obviously more exact than is actually necessary. A change of about this same magnitude results when one side of the secondary is raised by $1 \mathrm{~mm}$ while the opposite side is lowered by the same amount, i. e., when the secondary is rotated about one of its diameters. The greatest change occurs when this diameter is the one through the primary lead wire, but in no case is there any difficulty in making the secondary alinement to a sufficient accuracy.

(d) Uncertainty in Effective Value of $M$

The effect of errors in measuring the dimensions of the secondary are negligible. The apparent uncertainty in mean primary diameter is $\pm 2 \mathrm{ppm}$, whereas the uncertainty in mean pitch is $\pm 1 \mathrm{ppm}$. Ii these errors combine additively, the uncertainty in the value of $M_{0}$ may be as much as $5 \mathrm{ppm}$. An additional error of the same magnitude may be introduced because of uncertainties in the values of the several correction factors to be applied when the effective value of $M$ is calculated. Among the individual corrections, that for primary current distribution is most in doubt. The factors affecting current distribution have been discussed previously. An additional factor not covered in that analysis relates to skin effect. When the short-circuit is removed irom the primary, and the inductor is connected in series with the remainder of the circuit, the current in the primary winding of the inductor is brought to the same value as that in the remainder of the circuit, from which it initially differs by not more than 0.2 percent because of current mismatch. At first the distribution of this current across the section of the primary winding is not exactly the same as the direct-current distribution for which the mutual inductance was calculated. However, the current rapidly approaches the direct-current distribution during the series connection. Experimentally, the electrical balance of the measuring circuit was found not to change with the time of secondary commutation over most of the available range of the series connection. It is thereiore concluded that this change in current distribution is not appreciable. This effect is difficult to check by calculation, but is believed to be less than the change of the seliinductance of the primary winding with îrequency. Using an expression derived by Coffin [27], it was found that the seli-inductance at $25 \mathrm{c} / \mathrm{s}$ differs irom the direct-current value by about 2 parts in $10^{9}$.

It may be readily shown [28] that the current distribution is uniform to about 1 part in $10^{6}$ across the section of a long straight conductor of the size and material used for the primary helix when traversed by a $25-\mathrm{c} / \mathrm{s}$ current. When coiled into a helix the distribution is somewhat 
less uniform, because of the proximity effect of adjacent turns. Even so, the effect of this small departure from uniformity is negligible as compared with that which results from bending stresses in the wire. In this latter case a resistivity variation of \pm 0.4 percent over the section of the wire changed the mutual inductance by only a few parts in a million from that with a uniform distribution. These facts support the assumption that the effective value of mutual inductance is the same as that for a direct-current distribution.

The total error in $M$ may therefore be $\pm 10 \mathrm{ppm}$, but if some errors compensate one another, the probable uncertainty in $M$ may lie between 5 and $10 \mathrm{ppm}$. Over long periods of time, the mean dimensions of the inductor may change, but it is not likely that the values of the corrections will change materially. It is therefore believed that changes in $M$, as determined from dimensional measurements, can be determined within \pm 5 $\mathrm{ppm}$, and this is the figure of interest in connection with observations on the stability of the resistance unit maintained with wire standards.

\section{(e) Average Speed}

The instantaneous speed of the drive shaft is continually oscillating about a mean value at a frequency of 4 or 5 times per second. The mean speed over a given interval of time may be taken proportional to the quotient of the total angular travel divided by the length of the time interval. Obviously, if the time interval chosen is sufficiently long, the average speed over successive intervals of time will approach the constancy of the reference frequency. The time interval of interest is that which was referred to in section VIII, 4, as the galvanometer observing time. When balancing the measuring circuit, the galvanometer light spot moves back and forth over a range of several millimeters because of slight speed variations, which, however, are not sufficient to throw the system out of synchronism. The excursions of the light spot are integrated visually over a period of about a minute. During this period the total angular travel, measured on the 1,000-cycle reference system, is $60 \times 1,000 \times 360=21.6 \times 10^{6}$ electrical degrees, with a position uncertainty equal to the amplitude of hunting, which is about 30 electrical degrees as determined by stroboscopic observations. The speed averaged over the 1-min galvanometer observing time may therefore be uncertain by $\pm 1.5 \mathrm{ppm}$. The electrical circuit is balanced several times in order to determine the average value, $S$, of the shunt on $R$ (fig. 32), and the effect of repeated balances is to reduce the uncertainty in the average value of $n$ well below $1 \mathrm{ppm}$. The type of visual integration mentioned above can be made with surprising accuracy, as indicated by the fact that several observers working independently will usually agree within \pm 0.5 $\mathrm{ppm}$ on the value of $\mathrm{R}_{e}$ required to balance the circuit.

\section{(f) Stability of Primary Current}

Conditions relating to the proper matching of current in the two portions of the primary circuit have been discussed in section VIII, 3 . The discussion there considered the effect of voltage disturbances appearing across the reversing switch $C_{p}$. Voltage disturbances arising at that location may be thought of as alternating emf's having periods of $0.022 \mathrm{sec}$ or shorter. The vacuumtube chokes were designed to compensate these relatively rapid variations in emf, provided their amplitude was within certain limits, easily met. A question yet to be discussed arises in connection with the possibility of a change in primary current at a rate so slow that the stabilizing devices are ineffective. Such a change may be produced by a gradual increase in circuit resistance because of heating, or by a drift in terminal voltage of the main primary battery. It may be demonstrated analytically that the circuit balance is not disturbed if the current change is linear. The effect of a slow change in the value of the main primary current was tested in the following manner. An auxiliary load resistor of about 2 ohms was connected through a switch to the terminals of the main primary battery. This resistor was adjusted to draw 10 amp with the switch closed, and the effect of this load was to cause a gradual decrease in the terminal voltage of the battery and consequently a decrease in the value of the test current. The test current was measured continuously with a potentiometer. The test was begun by allowing the apparatus to warm up for a 20-min period with the auxiliary load switch open. The currents in the two portions of the primary circuit were then matched as closely as possible and the circuit balanced in the usual manner. The balance was then observed for a 30-min period, the load switch being closed for 
the first $15 \mathrm{~min}$ and open for the last $15 \mathrm{~min}$. During this run the primary current decreased gradually by 2 ma during the first 15 -min period and rose again by practically the same amount during the last $15 \mathrm{~min}$. No definitely measurable change occurred in the balance of the main circuit during this run. The current match in the two parts of the primary circuit did not remain perfect during this test, but the deviations were such that no error exceeding $0.5 \mathrm{ppm}$ could have arisen from this cause.

If the primary current rises exponentially from one steady-state level to another, the balance of the circuit will suffer a transient disturbance, a decreasing current producing a disturbance of opposite sign. Such exponential changes in current are produced by discontinuous changes in circuit resistance or battery terminal voltage. The effect was tested by inserting a resistance of about $0.2 \mathrm{ohm}$ in series with $R h$ of figure 32 . By opening and closing a shorting switch connected across this resistance, the effect produced was the equivalent of a sudden decrease or increase of $0.16 \mathrm{v}$ in battery terminal voltage. After balancing the circuit, the auxiliary switch was opened. This produced a sudden change of about $5 \mathrm{~mm}$ in the galvanometer deflection, after which the galvanometer spot soon returned to its original position. A 5-mm change in galvanometer deflection is equivalent to a 5-ppm change in the value of the resistance required to balance the circuit. When the auxiliary switch was closed the opposite effect was observed.

A cathode-ray oscillograph equipped with a suitable amplifier was connected across the terminals of the main primary battery in order to determine what sudden voltage variations occurred. No sudden variations as great as $0.003 \mathrm{v}$ were observed. There are no circuit elements between $B_{1}$ and $C_{p}$ of figure 32 that would produce sudden resistance changes as great as $0.01 \mathrm{ohm}$. The possible discontinuous changes in resistance and voltage are therefore over one order less than that artificially produced in the test described above.

All the tests discussed in this section support the conclusion that no error as great as $1 \mathrm{ppm}$ can exist because of instability of primary current.

(g) Shorting of Secondary During Commutation

Under ideal conditions the inductor generator in the secondary circuit would furnish an emf that would exactly neutralize the remaining emf's in the secondary circuit when the condition of balance is attained, and no current would flow at any time in the secondary circuit. Starting from this ideal balance, a small change in the shunt on the primary resistor would produce a small unbalanced direct-current emf, which would cause a current to flow through the galvanometer, indicating the lack of balance.

Under theoretical conditions, then, the current in the secondary circuit would be zero at balance, and no disturbance in it would be caused by the short-circuiting of the secondary of the mutual inductor that occurs during commutation. Moreover, when the circuit is slightly out of balance in either direction, the effect of shorting would be to produce a small change in the value of the galvanometer current, but this would not shift the experimental setting of the resistance for a balance.

The effect of this short-circuiting of the secondary of the inductor may be readily approximated sufficiently well for present purposes. The secondary circuit has a resistance of about $650 \mathrm{ohms}$ and "choke coils" of $2,000 \mathrm{~h}$ of inductance. The secondary of the mutual inductor has a resistance of about $15 \mathrm{ohms}$ and a self-inductance of about $0.05 \mathrm{~h}$. The short-circuit at the time of reversal of the secondary connections lasts about $2 \mathrm{~ms}$. Suppose the circuit is out of balance because the resistor ( $R$ of fig. 1 ) is high by $1 \mathrm{ppm}$. This will introduce in the secondary circuit a direct-current emf of about $1 \mu \mathrm{v}$, which will cause a current, $i_{s}$, of approximately $0.0015 \mu$ a through the secondary circuit. This current will be flowing through the secondary of the mutual inductor as it is shorted. The time constant of the shorted secondary is such that the current through it will drop to about half its initial value, i. e., from 0.0015 to $0.0008 \mu \mathrm{a}$, during the time of the shortcircuit. At the end of the short, the tremendous inductance of the chokes will almost immediately restore the current to $0.0015 \mu \mathrm{a}$, but in the opposite direction. The change in current, $\Delta i_{s}$, is then $0.0015+0.0008 \mu \mathrm{a}$, and since this takes place twice per cycle, the average induced emf, $E_{s}$, in the secondary of the inductor is

$$
\begin{aligned}
E_{s}=L \frac{\Delta i_{s}}{1 / n} & =0.05 \times 0.0046 \times 10^{-6} \times 23 \\
& =0.005 \mu \mathrm{v} .
\end{aligned}
$$


As compared with the unbalanced emf of $1 \mu \mathrm{v}$, which corresponds to an unbalance of about $1 \mathrm{ppm}$ in the measuring circuit, $E_{s}$ is obviously negligible. The direction of $E_{s}$ is such as to reduce the current in the secondary circuit.

In addition to the effect caused by $E_{s}$, the shortcircuit causes a temporary decrease in the'resistance of the secondary circuit, by the amount of the resistance of the leads and of the secondary winding of the mutual inductor. The effect on the secondary current is the same as if an emf, of average value $E_{s}{ }^{\prime}$, were introduced into the secondary circuit. The value of this equivalent emf is approximately

$$
\begin{aligned}
E_{s}^{\prime} & =\frac{t}{T} r i_{s} \\
& =0.1 \times 15 \times 1.5 \times 10^{-9} \mathrm{v} \\
& =0.0023 \mu \mathrm{v},
\end{aligned}
$$

where $t / T$ is the proportion of the time the circuit is shorted, and $r$ is the value of the shorted resistance.

It should be noted that $E_{s}{ }^{\prime}$ and $E_{s}$ act in opposition and tend to neutralize one another. In fact, $E_{s}{ }^{\prime}$ could be increased by means of a series resistor until $E_{s}+E_{s}{ }^{\prime}=0$, which would have been done if the two effects had not been negligible.

Although the above discussion of the effect of short-circuiting the secondary applies only to the theoretical case of perfect operation, the actual operation was found to be sufficiently near the ideal that the effect is negligible. This was tested by separately doubling both the inductance and the resistance of the circuit of the shorted secondarv winding. In neither case was there a detectable change in the balance of the main circuit.

If the inductor generator were not used in the secondary circuit, the emf in the secondary circuit at balance would be a square-topped wave of about 1-v peak value. This would cause an alternating current through the secondary circuit whose fundamental would have a peak value of about $2 \mu \mathrm{a}$ and which would lag behind the emf by nearly $90^{\circ}$. This current would then go through zero during the time of the short-circuit of the secondary. How large the average induced emf would be because of the self-inductance of the secondary winding would depend upon the magnitude of the secondary current at the start and end of the short-circuit. A balance of the main circuit made with and without the inductor generator gave results that differed by $3 \mathrm{ppm}$. If the inductor generator had not been provided (to aid the galvanometer to integrate correctly) it would have been necessary to adjust a resistance in series with the secondary winding in order to render negligible the effect of short-circuiting the secondary.

\section{(h) Stray Electromotive Forces}

One of the advantages of this method of measuring resistance in absolute units is the fact that the rectified secondary emf is large. The stray thermal emf's in the secondary commutator are therefore small in comparison. In addition, the commutator was designed to minimize such thermal emf's, and the test procedure eliminates their effect insofar as they remain constant during successive observations with the primary test current reversed.

It is necessary that no coupling occur between the primary and secondary circuits except via the mutual inductance between the primary and secondary windings of the inductor. Hence the secondary leads from the inductor are twisted and well separated from the primary leads, which are also twisted. In the vicinity of the commutators the secondary leads are carried in an iron conduit, and the secondary commutator is enclosed in a steel box. The inductor is located at a. distance of about $20 \mathrm{~m}$ from the commutators, and the latter are about $2 \mathrm{~m}$ from the driving motor and the loading generator.

It is also desirable that all other-nonreversible components of direct current emf be minimized. These components arise from thermal emf's at junctions in the secondary circuit, induction caused by rotation of segments of the secondary circuit (in the secondary commutator) in the earth's field, and synchronous emf's arising from sources other than the primary test current. These nonreversible emf's are minimized by the spacing and shielding mentioned above; and small residuals are eliminated during the measurement by taking balances with the primary test current reversed.

\section{(i) Scattering of Results}

The curve in figure 43 has been drawn as the most representative smooth curve connecting the 15 plotted points covering the $10-\mathrm{yr}$ period during which absolute measurements have been made by the Wenner method. Each plotted 
point is, in general, the mean of a large number of observations, as shown in figure 42. The mean deviation from the mean for July 1941 is $0.4 \mathrm{ppm}$. If the scattering had been entirely random, it would be reasonable to expect that the mean for adjacent months would deviate from the smooth curve by less than this amount, but this was found to be not so, the mean for June 1941 being $1.5 \mathrm{ppm}$ below the smooth curve as drawn. A considerable body of evidence, for which there is, however, no definite proof, seems to indicate that the dimensions of the inductor are varying slightly from month to month in an erratic fashion, perhaps because of dimensional hysteresis, with a net long-time increase as indicated by the smooth curve. In spite of the great effort spent in designing and constructing the mutual inductor, it apparently does not possess a stability equal to that of the best present-day large double-walled sealed standard resistors.

An extended series of dimensional measurements was made on the inductor between December 1947, and June 1948. The calculated value of $M$ arrived at on the basis of these measurements is considered to apply to the inductor on the median date, March 1948. The inductor was not available for electrical measurements on that date, but the resistance that would have been required to balance the circuit at that time is taken from the smooth curve of figure 43 . This value of $R_{e}$ may perhaps be in error by $1 \mathrm{ppm}$, because the exact form of the curve between adjacent electrical observations is unknown. The same considerations apply to the first dimensional measurements made in December 1938.

\section{Estimate of Over-All Accuracy}

A large number of factors must be taken into account in estimating the accuracy of the results of an investigation of this type. Some of these factors enter in a perfectly random way, and errors from them may be reduced by taking a large number of observations. Certain errors, however, are not random, and for others a large number of observations are not practicable. It is therefore impossible, on a statistical basis, to assign a "probable error" to the final result. A statement of the over-all accuracy is primarily an estimate on the part of the authors of how well systematic errors have been reduced. It is the opinion of the authors that in this investigation random errors have been reduced to such an extent that systematic errors predominate.

Probably the greatest potential source of error is the mutual inductor, whose value is believed to be known to be between 5 and $10 \mathrm{ppm}$. In spite of the large number of observations, uncertainties in temperature during the measurement of the mechanical dimensions of the primary may have caused errors amounting to 4 or $5 \mathrm{ppm}$. Errors of equal magnitude may result from a lack of knowledge of the exact value of various corrections, particularly that for current distribution in the wire of the primary helix. The design of the inductor is such that improper alinement of the leads or of the secondary coil should not have caused an error exceeding $1 \mathrm{ppm}$.

The speed control has been remarkably satisfactory, and the value of frequency of reversal of the primary current is believed to be reliable to better than $1 \mathrm{ppm}$. The only remaining source of error is in the measuring circuit. Here also the performance has been entirely satisfactory, and no known source of appreciable error remains. It is probable that the average of several readings is in error by 1 ppm at the most.

Summarizing, it is believed that the unit of resistance as maintained at this Bureau has been measured in absolute units to $\pm 10 \mathrm{ppm}$, and perhaps somewhat better. It is probable that the uncertainty could be reduced to 2 or $3 \mathrm{ppm}$ by the construction of a more nearly perfect mutual inductor and by an improvement of the temperature control during electrical and dimensional measurement. If the primary were wound on a Pyrex or fuzed quartz form a more uniform coil could probably be obtained. The uncertainty caused by the nonuniform current distribution in the primary winding could be reduced considerably by using a smaller wire. This would increase the heating of the primary by the test current, but the effect of this heating could be measured electrically.

This method of measuring resistance could be used for routine calibrations of 1-ohm standards to a few parts in a million in terms of inductance and frequency. The principal difficulties would arise from the fact that the average standard is not designed to carry currents as large as that used in the measuring circuit and that investigations of the lead resistances of the standards would be required. 


\section{Summary}

An 11-mh mutual inductor, of a modified Campbell type, has been constructed, and its value has been determined to a few parts in a million in terms of its measured dimensions. Using a direct current commutated at a known frequency, this inductor has been used to determine the resistance of a standard resistor in terms of the calculated mutual inductance and time. The value of resistance so determined is believed to have been correct to 10 parts per million. These results indicate that the ohm as now maintained at this Bureau with wire-wound resistors is equal to 0.999994 absolute ohm. The values for these resistors were assigned on the basis of the value of 1.000490 for the ratio between the mean international and the absolute ohm as selected in 1946 by the Advisory Committee on Electricity of the International Committee on Weights and Measures. This ratio was based upon experimental determinations at several national standardizing laboratories.

The dimensions of the mutual inductor were determined in 1938 and again in 1948. After allowing for the change in the value of inductance caused by drifts in the dimensions of the inductor, the average resistance of the ten standard resistors used to maintain the unit at this Bureau was found to be the same in 1948 as in 1938 within 1 part in a million. Even allowing for possible errors of a few parts in a million in the dimensional determinations, this is the first satisfactory method that has been devised for independently checking the stability of the unit as maintained by a group of wire-wound resistors. In the future repetitions of the dimensional and electrical measurements should greatly increase the assurance in the maintenance of the ohm.

The authors acknowledge the contributions of Frank Wenner to this project. The preliminary determination was carried through under his direction. To his genius must be credited much of the technique that has made this method, in our opinion, the best yet devised for the absolute measurement of electrical resistance.

\section{References}

[1] Henry A. Rowland, Research on the absolute unit of resistance, Am. J. Sci. 115, 430 (1878).

[2] H. L. Curtis, Review of recent determinations of the ohm and the ampere, J. Research NBS 33, 235 (1944) RP1606.

[3] F. Wenner, A proposed modification of the Kirchoff method for the absolute measurement of resistance, Science 29, 475 (1909).

[4] Frank Wenner, Time measurements, J. Wash. Acad. Sci. 28, 505 (1938).

[5] I. L. Cooter, F. Wenner, and C. Peterson, A vacuumtube alternating voltage compensator, J. Research NBS 25, 41 (1940) RP1312.

[6] A. Campbell, On a standard of mutual inductance, Proc. Roy. Soc. (London) [A] 79, 428 (1907).

[7] H. L. Curtis, C. Moon, and C. M. Sparks, A determination of the absolute ohm, using an improved self-inductor, J. Research NBS 21, 375 (1938) RP1137.

[8] J. Viriamu Jones, On the calculation of the coefficient of mutual induction of a circle and a coaxial helix, etc., Proc. Roy. Soc. (London) 63, 192 (1898).

[9] L. V. King, On the direct numerical calculation of elliptic functions and integrals (Cambridge University Press, 1924). (There is a typographical error in the coefficient of the general term in eq 75).

[10] Walter Bartky, Numerical calculation of generalized complete elliptic integrals, Rev. Modern Phys. 10, 264 (1938).

[11] Nagaoka and Sakurai, Tables for facilitating the calculation of self-inductance of circular coil and of mutual inductance of coaxial circular currents, The Institute of Physical and Chemical Research, Tokyo (1927).

[12] P. W. Bridgman, The electrical resistance of metals under pressure. Proc. Am. Acad. Arts Sci. 52, 537 (1917).

[13] F. R. Kotter, Master's thesis, George Washington University (1940).

[14] A. E. H. Love, A treatise on the mathematical theory of elasticity (Cambridge University Press, 1934, 4th ed., p. 129, articles 87 and 88).

[15] J. C. Maxwell, Electricity and magnetism 2, sec. 701.

[16] E. B. Rosa and F. W. Grover, Formulas and tables for the calculation of mutual and self-inductance (Revised). BS Sci. Pap. 8, 1 (1912) S169.

[17] James L. Thomas, Stability of double-walled manganin resistors, J. Research NBS 36, 107 (1946). RP1692.

[18] James L. Thomas, Precision resistors and their measurement, NBS Circular 470 (1948).

[19] Comité International des Poids et Mesures, Procèsverbaux des séances, 2e Série, TXIX-1939, E48.

[20] L. Hartshorn and N. F. Astbury, The absolute measurement of resistance by the method of Albert Campbell, Trans. Roy. Soc. (London) [A] 236, 423 (1937). 
[21] P. Vigoureux, Determination of the ohm by the method of Lorenz, Ntl. Phys. Lab. Collected Researches $\mathbf{2 4}, 279$ (1938).

[22] R. Jouaust, M. Picard, R. Hérou, Determination of the unit of resistance in the cgs electromagnetic system, Bul. Soc. franç. élec. [5] 8, 587 (1938).

[23] Comité International des Poids et Mesures, Procèsverbaux des séances, 2e Série, TXIX (1939), E68.

[21] Comité International des Poids et Mesures, Procèsverbaux des séances, 2e Série, TXVIII (1937), 178.

[25] Comité International des Poids et Mesures, Procèsverbaux des séances, 2e Série, T XIX (1939) E41.
[26] H. R. Nettleton and E. G. Balls, The absolute measurement of electrical resistance by a method using the average electromotive force of a commutating generator, Proc. Phys. Soc. (London) 54, 27 (1942).

[27] J. G. Coffin, Bul. BS 2, 289 (1906) S37.

[28] Lord Kelvin, Mathematical and physical papers III, 491 (Cambridge Univ. Press, 1890).

Washington, April 12, 1949. 